\title{
LS(Graph): A Constraint-Based Local Search for Constraint Optimization on Trees and Paths
}

\author{
Pham Quang Dung • Yves Deville • Pascal Van \\ Hentenryck
}

Received: date / Accepted: date

\begin{abstract}
Constrained optimum tree (COT) and constrained optimum path (COP) problems arise in many real-life applications and are ubiquitous in communication networks. They have been traditionally approached by dedicated algorithms, which are often hard to extend with side constraints and to apply widely. This paper proposes a constraint-based local search framework for COT/COP applications, bringing the compositionality, reuse, and extensibility at the core of constraint-based local search and constraint programming systems. The modeling contribution is the ability to express compositional models for various COT/COP applications at a high level of abstraction, while cleanly separating the model and the search procedure. The main technical contribution is a connected neighborhood based on rooted spanning trees to find high-quality solutions to COP problems. This framework is applied to some COT/COP problems, e.g., the quorumcast routing problem, the edge-disjoint paths problem, and the routing and wavelength assignment with delay side constraints problem. Computational results show the potential importance of the approach.
\end{abstract}

Pham Quang Dung

Hanoi University of Science and Technology

School of Information and Communication Technology, Hanoi, Vietnam

Tel.: (+84)43 8692463

Fax: (+84)438692906

E-mail: dungpq@ soict.hut.edu.vn

Yves Deville

Université catholique de Louvain B-1348 Louvain-la-Neuve, Belgium

Tel: (++32)10472067

Fax: (++32)10450345

E-mail: yves.deville@uclouvain.be

Pascal Van Hentenryck

Optimization Research Group, NICTA, Victoria Research Laboratory, Electrical and Electronic Engineering,

The University of Melbourne, VIC 3010, Australia

E-mail: pvh@nicta.com.au 


\section{Introduction}

Constrained optimum tree (COT) and constrained optimum path (COP) problems appear in various real-life applications such as telecommunication and transportation networks. These problems consist of finding one or more trees (or paths) on a given graph satisfying some given constraints while minimizing or maximizing an objective function. Some COT problems have been considered and solved in the literature, e.g., Degree Constrained Minimum Spanning Tree (DCMST) [45, 7], Bounded Diameter Minimum Spanning Tree (BDMST) [35], Capacitated Minimum Spanning Tree problem (CMST) [56,3], Minimum Diameter Spanning Tree (MDST) [50], EdgeWeighted $k$-Cardinality Tree (KCT), [20,25], Steiner Minimal Tree (SMT) [66,28], Optimum Communication Spanning Tree problems (OCST) [32], etc. We also see many COP problems which have been studied and solved in the literature. For instance, in telecommunication networks, routing problems supporting multiple services involve the computation of paths minimizing transmission costs while satisfying bandwidth and delay constraints $[15,27,30]$. Similarly, the problem of establishing routes for connection requests between network nodes is one of the basic operations in communication networks and it is typically required that no two routes interfere with each other due to quality-of-service and survivability requirements. This problem can be modeled as an edge-disjoint paths problem [18]. Most of these COT/COP problems are NP-hard. They are often approached by dedicated algorithms including exact methods, such as the Lagrangian-based heuristic [7], the ILP-based algorithm using directed cuts [25], the Lagrangian-based branch and bound in [15], and the vertex labeling algorithm from [30]; there are also meta-heuristic algorithms such as a hybrid evolutionary algorithm [19], ant colony optimization [21], and local search [20]. These techniques exploit the structure of the constraints and the objective functions but are often difficult to extend or reuse.

This paper ${ }^{1}$ proposes a constraint-based local search (CBLS) [62] framework for COT/COP applications to support the compositionality, reuse, and extensibility at the core of CBLS and CP systems. It follows the trend of defining domain-specific CBLS frameworks, capturing modeling abstractions and neighborhoods for classes of applications exhibiting significant structures. As is traditional for CBLS, the resulting LS (Graph) framework allows the model to be compositional and easy to extend, and provides a clean separation of concerns between the model and the search procedure. Moreover, the framework captures structural moves that are fundamental in obtaining high-quality solutions for COT/COP applications. The key technical contribution underlying this COP framework is a novel connected neighborhood for COP problems based on rooted spanning trees. More precisely, this COP framework incrementally maintains, for each desired elementary path, a rooted spanning tree that specifies the current path and provides an efficient data structure to obtain its neighboring paths and their evaluations.

The availability of high-level abstractions (the "what") and the underlying connected neighborhood for elementary paths (the "how") make the LS (Graph) framework particularly appealing for modeling and solving complex COP applications.

1 This paper is an extended version of [54] and is based on the PhD thesis [53]. 
The LS (Graph) framework, implemented in COMET, was evaluated experimentally on two classes of applications: COT with the quorumcast routing $(\mathrm{QR})$ problem and $\mathrm{COP}$ with the edge-disjoint path (EDP) problems and the routing and wavelength assignment problem with side constraints (RWA-D). In [37], we present another application in the domain of traffic engineering in switched ethernet networks. The experimental results show the potential of the approach.

\subsection{Case studies}

We first describe three problems that will be modeled and solved by the LS (Graph) framework.

\subsubsection{The quorumcast routing $(Q R)$ problem}

The quorumcast routing $(\mathrm{QR})$ problem arises in distributed applications [24,29,63, 48]. Given a weighted undirected graph $G=(V, E)$, to each edge $e \in E$ there is associated a cost $w(e)$. Given a source node $r \in V$, an integral value $q$, and a set $S \subseteq V$ of multicast nodes, the quorumcast routing problem consists in finding a minimum cost tree $T=\left(V^{\prime}, E^{\prime}\right)$ of $G$ spanning $r$ and $q$ nodes of $S . T=\left(V^{\prime}, E^{\prime}\right)$ is a graph satisfying the following properties:

1. $V^{\prime} \subseteq V \wedge E^{\prime} \subseteq E$.

2. $T$ is connected.

3. $\exists Q \subseteq S$ such that $\sharp Q=q \wedge Q \cup\{r\} \subseteq V^{\prime}$.

4. The cost of

$$
T=\sum_{e \in E^{\prime}} w(e)
$$

is minimal over all subgraphs of $G$ with properties 1,2 , and 3 .

An exact algorithm [48] has also been proposed for solving the QR problem but experiments were performed on small graphs (e.g., graph with 30 nodes). Three heuristics have been proposed in [24] including Minimal Cost Path Heuristic (MPH), Improved Minimum Path Heuristic (IMP), and Modified Average Distance Heuristic (MAD). Experimental results in that paper show that, among these heuristics, the IMP heuristic produces the best solutions. In [29], a multispace search heuristic has been proposed for solving this problem which gives better results than the IMP and the MAD heuristics on 12-node networks and 100-node networks.

In [63], the authors considered the $\mathrm{QR}$ problem with additional constraints imposed on the total cumulative delay along the path from $s$ to any destination node of $Q$, and proposed a distributed heuristic algorithm for solving it. Experiments were conducted on graphs of up to 200 nodes.

In Section 6.1, we propose a simple model in LS (Graph) for this problem using a tabu search. This example illustrates the expressive power of LS (Graph) where a simple but efficient model can be designed in a few lines. Experimental results show that our LS (Graph) model gives better results than the standard IMP heuristic. 


\subsubsection{The edge-disjoint paths (EDP) problem}

We are given an undirected graph $G=(V, E)$ and a set $T=\left\{\left\langle s_{i}, t_{i}\right\rangle \mid i=\right.$ $\left.1,2, \ldots, \sharp T ; s_{i} \neq t_{i} \in V\right\}$ representing a list of commodities. A subset $T^{\prime} \subseteq T$, $T^{\prime}=\left\{\left\langle s_{i_{1}}, t_{i_{1}}\right\rangle, \ldots,\left\langle s_{i_{k}}, t_{i_{k}}\right\rangle\right\}$ is called $e d p$-feasible if there exist mutually edgedisjoint paths from $s_{i_{j}}$ to $t_{i_{j}}$ on $G, \forall j=1,2, . ., k$. The EDP problem consists in finding a $e d p$-feasible subset of $T$ with maximal cardinality. In other words,

$$
\begin{array}{lll}
\max & \sharp T^{\prime} & \text { (1) } \\
\text { s.t. } & T^{\prime} \subseteq T & \text { (2) } \\
& T^{\prime} \text { is } e d p \text {-feasible }
\end{array}
$$

This problem appears in many applications such as real-time communication, VLSI-design, routing, and admission control in modern networks [8,23]. The existing techniques for solving this problem include approximation algorithms [43, 13,42,22], greedy approaches $[42,44]$, and an ant colony optimization (ACO) metaheuristic [18]. It has been shown in [18] that ACO is the start-of-the-art algorithm for this problem. In that paper, the ACO algorithm were compared with a simple greedy algorithm in [42](the multi-start version).

In Section 6.2, we propose two heuristic algorithms applying LS (Graph). We experimentally show competitive results compared with the ACO algorithm in [18]. This example illustrates how LS (Graph) can be used to implement more complex heuristics.

\subsubsection{The routing and wavelength assignment problem with a delay side constraint (RWA-D)}

Wavelength division multiplexing (WDM) optical networks [49] provide high bandwidth communications. The routing and wavelength assignment (RWA) problem is an essential problem on WDM optical networks. The RWA problem can be described as follows. Given a set of requests for all-optical connections, the RWA problem consists of finding routes from the source nodes to their respective destination nodes and assigning wavelengths to these routes. A condition that must be satisfied is that two routes sharing common edges must be assigned different wavelengths. Normally, the number of available wavelengths is limited and the number of requests is high. Two variants of this problem have been studied extensively in the literature: the min$R W A$ problem aims at minimizing the number of wavelength used for satisfying all requests, and the $\max R W A$ aims at maximizing the number of requests with a given number of wavelengths. Both variants are NP-Hard [26].

In the literature, there have been different techniques proposed for solving these problems, e.g.: exact methods based on the ILP formulation $[23,46,61,52,55,47,39$, 65]; heuristic algorithms [31,67,11,12]; and metaheuristics, including tabu search $[40,51]$ and Genetic $[4,10,38]$. These techniques have been tried on realistic networks of small size (networks up to 27 nodes and 70 edges) but involving a large number of connection requests. RWA with additional constraints has also been considered, e.g., in $[64,5]$.

In order to show the interest of the modeling framework, we consider the minRWA problem with a side constraint (e.g., a delay constraint) specifying that the cost of 
each route must be less than or equal to a given value. The point here is not to study a model competitive in comparison with state-of-the-art techniques for classical RWA problems. Rather, we show the flexibility of this modeling framework, one which enables a combination of VarGraph of LS (Graph) with var\{int $\}$ of COMET.

The formal definition of the problem (called RWA-D) is the following. Given an undirected weighted graph $G=(V, E)$, each edge $e$ of $G$ has cost $c(e)$ (e.g., the delay in traversing $e$ ). We suppose given a set of connection requests $R=$ $\left\{\left\langle s_{1}, t_{1}\right\rangle,\left\langle s_{2}, t_{2}\right\rangle, \ldots,\left\langle s_{k}, t_{k}\right\rangle\right\}$ and a value $D$. The RWA-D problem consists of finding routes $p_{i}$ from $s_{i}$ to $t_{i}$ and their wavelengths for all $i=1,2, \ldots, k$ such that:

1. the wavelengths of $p_{i}$ and $p_{j}$ are different if they have common edges, $\forall i \neq j \in$ $\{1,2, \ldots, k\}$ (wavelength constraint),

2. $\sum_{e \in p_{i}} c(e) \leq D, \forall i=1,2, \ldots, k$ (delay constraint)

3 . the number of different wavelengths is minimized (objective function).

In Section 6.3, a local search algorithm and its implementation in LS (Graph) will be proposed for solving the RWA-D problem.

\subsection{Contribution}

The contributions of this paper are the following:

1. We design and implement a constraint-based local search (CBLS) [62] framework, called LS (Graph), for COT/COP applications. It supports the compositionality, reuse, and extensibility at the core of CBLS and CP systems. The proposed framework can be used as either a black box or a glass box. The black box is exploited in the sense that users only need to state the model in a declarative way, with variables, constraints, and an objective function to be optimized. Built-in search components (e.g., tabu search) are then performed automatically. The glass box allows users to extend the framework by designing and implementing their own components (e.g., invariants, constraints, objective functions, and search heuristics) and integrating them with the system.

2. The LS (Graph) combines graph variables (i.e., VarTree, VarPath for modeling trees and paths in a high-level way) with standard var $\{$ int $\}$ of COMET, which enables the modeling of various COT/COP applications on graphs for which both the topology and scalar values must be determined.

3. A key technical contribution of the paper is a novel connected neighborhood for COP problems based on rooted spanning trees. More precisely, the COP framework incrementally maintains, for each desired elementary path, a rooted spanning tree that specifies the current path and provides an efficient data structure to obtain its neighboring paths and their evaluations.

4. We propose incremental algorithms for implementing some fundamental abstractions of the framework. We show that the incrementality does not improve the theoretical complexity but is efficient in practice.

5. We apply the constructed framework to a COT problems: the quorumcast routing problem and two COP problems: the edge-disjoint paths problem and the routing 
and wavelength assignment problem with delay side constraints on optical networks. Experimental results show the potential significance of our approach from both the programming and the computation stand points. For the first two problems, we show competitive results in comparison with existing techniques and for the third problem, we show how to solve complex problems flexibly and easily.

The LS (Graph) framework is open source. The COMET code of LS (Graph) and applications as well as instances experimented in this paper are available at

http://becool.info.ucl.ac.be/lsgraph

\subsection{Outline}

The rest of this paper is organized as follows. Section 2 gives the basic definitions and notations. Section 3 specifies neighborhoods for COT applications and proposes our novel neighborhoods for COP applications. Section 4 gives an overview of data structures and algorithms for implementing two fundamental and non-trivial abstractions of the framework. The implementation of the framework in COMET programming language will be introduced in Section 5. Sections 6 presents the application of the framework to the resolution of the QR, EDP and RWA-D problems. Finally, Section 7 concludes the paper and gives some future work.

\section{Definitions and Notations}

Graphs Given an undirected graph $g$, we denote the set of nodes and the set of edges of $g$ by $V(g), E(g)$ respectively. The degree of a node $v$ (denoted $\operatorname{deg}_{g}(v)$ ) is the number of incident edges to this edge: $\operatorname{deg}_{g}(v)=\sharp\{u \mid(v, u) \in V(g)\}$.

A graph $s g$ is called subgraph of a graph $g$ if $V(s g) \subseteq V(g)$ and $E(s g) \subseteq E(g)$ and we denote $s g \subseteq g$.

A path on $g$ is a sequence of nodes $\left\langle v_{1}, v_{2}, \ldots, v_{k}\right\rangle(k>1)$ in which $v_{i} \in V(g)$ and $\left(v_{i}, v_{i+1}\right) \in E(g), \forall i=1, \ldots, k-1$. The nodes $v_{1}$ and $v_{k}$ are the origin and the destination of the path. A path is called simple if there is no repeated edge and elementary if there is no repeated node. A cycle is a path in which the origin and the destination are the same. This paper only considers elementary paths and hence we use "path" and "elementary path" interchangeably if there is no ambiguity. A graph is connected if and only if there exists a path from $u$ to $v$ for all $u, v \in V(g)$.

Given two paths $p x=\left\langle x_{1}, x_{2}, \ldots, x_{k}\right\rangle$ and $p y=\left\langle y_{1}, y_{2}, \ldots, y_{q}\right\rangle$, we denote $p x+$ $p y$ the concatenation of these two paths: $p x+p y=\left\langle x_{1}, x_{2}, \ldots, x_{k}, y_{1}, y_{2}, \ldots y_{q}\right\rangle$ if $x_{k} \neq y_{1}$ and $p x+p y=\left\langle x_{1}, x_{2}, \ldots, x_{k}=y_{1}, y_{2}, \ldots, y_{q}\right\rangle$ if $x_{k}=y_{1}$.

Given paths $p, p_{1}, p_{2}$, and $q$,

- $V(p)$ is the set of nodes of $p$

- $p_{1} \cup p_{2}\left(p_{1} \cap p_{2}\right)$ is the set $V\left(p_{1}\right) \cup V\left(p_{2}\right)\left(V\left(p_{1}\right) \cap V\left(p_{2}\right)\right)$.

- $x \in P$ is the predicate $x \in V(p)$.

- $s(p), t(p)$ are, respectively, the starting and terminating nodes of $p$. 
- $p(u, v)$ is the subpath of $p$ starting from $u$ and terminating at $v(u, v \in p$ and $u$ is not located after $v$ on $p$ ).

- $s p_{p}(x), t p_{p}(x)$ is the subpath of $p$ from $s(p)$ to $x$ and from $x$ to $t(p)$.

- $\operatorname{repl}(p, q)=s p_{p}(s(q))+q+t p_{p}(t(q))$ with $s(q), t(q) \in p$. Intuitively, $\operatorname{repl}(p, q)$ is the path generated by replacing the subpath of $p$ from $s(q)$ to $t(q)$ by $q$.

Trees A tree is an undirected connected graph containing no cycles. A spanning tree $t r$ of an undirected connected graph $g$ is a tree spanning all the nodes of $g: V(t r)=$ $V(g)$ and $E(t r) \subseteq E(g)$. A tree $t r$ is called a rooted tree at $r$ if the node $r$ has been designated the root. Each edge of $t r$ is implicitly oriented towards the root. If the edge $(u, v)$ is oriented from $u$ to $v$, we call $v$ the father of $u$ in $t r$, which is denoted by $f a_{t r}(u)$. Given a rooted tree $t r$ and a node $s \in V(t r)$,

- $\operatorname{root}(t r)$ denotes the root of $t r$,

- $\operatorname{path}_{t r}(v)$ denotes the path from $v$ to $\operatorname{root}(t r)$ on $t r$. For each node $u$ of $p_{t} h_{t r}(v)$, we say that $u$ dominates $v$ in $\operatorname{tr}$ (alternatively, $u$ is a dominator of $v, v$ is a descendant of $u$ ) which we denote by $u D_{\text {om }} v$. If $u$ does not dominates $v$ on $t r$, we write $u \overline{D o m}_{t r} v$.

- $\operatorname{path}_{t r}(u, v)$ denotes the path from $u$ to $v$ in $\operatorname{tr}(u, v \in V(t r))$.

- $n c a_{t r}(u, v)$ denotes the nearest common ancestor of two nodes $u$ and $v$. In other words, $n c a_{t r}(u, v)$ is the common dominator of $u$ and $v$ such that there is no other common dominator of $u$ and $v$ that is a descendant of $n c a_{t r}(u, v)$.

- Given a node $v \in V(t r)$, we denote by $T_{t r}(v)$ the subtree of $t r$ rooted at $v$. If $v \neq \operatorname{root}(t r)$, we denote by $\overline{T_{t r}}(v)$ the subtree of $t r$ generated by removing $T_{t r}(v)$ and the edge $\left(v, f a_{t r}(v)\right)$ from $t r: V\left(\overline{T_{t r}}(v)\right)=V(t r) \backslash V\left(T_{t r}(v)\right)$ and $E\left(\overline{T_{t r}}(v)\right)=E(t r) \backslash\left(E\left(T_{t r}(v)\right) \cup\left\{\left(v, f a_{t r}(v)\right)\right\}\right)$.

Property 1 Suppose given a rooted tree $t r$.

1. Suppose given a node $x \in V(t r)$. We have $x D_{0 m_{t r}} y, \forall y \in V\left(T_{t r}(x)\right)$. In other words, a vertex $x$ of a rooted tree $t r$ dominates all vertices of the subtree of $t r$ rooted at $x$.

2. Suppose given two nodes $x, y \in V(t r)$ such that $x=f a_{t r}(y)$ and two nodes $z, v$ such that $z \in V\left(T_{t r}(y)\right), v \in V\left(\overline{T_{t r}}(y)\right)$. We have $n c a_{t r}(v, z)=n c a_{t r}(v, x)$. This property is illustrated in Figure 1: $n c a_{t r}(v, z)=n c a_{t r}(v, x)=12$.

\section{Neighborhoods}

This section defines neighborhoods for COT and COP problems. The neighborhood for COT applications is based on traditional modification actions on dynamic trees (i.e., trees which can be modified): add, remove, and replace over edges. Our main technical contribution for COP applications is to propose a neighborhood structure based on spanning trees. We first present neighborhoods for COT applications. 


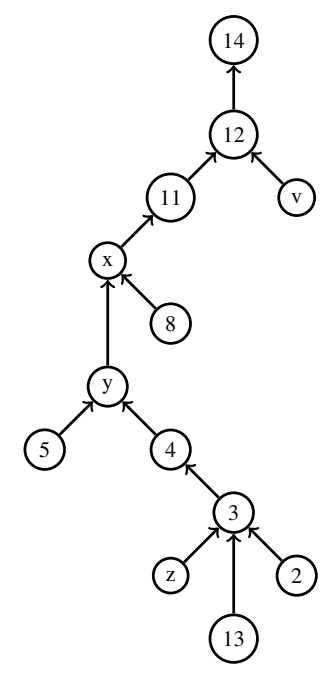

Fig. 1 Illustrating property 1

\subsection{COT neighborhood}

A neighborhood of a tree is a set of trees generated by performing modification actions on the given tree. Given an undirected graph $g$ and a dynamic tree $t r$ of $g$ ( $t r$ can be modified such that $t r \subseteq g$ ), we specify a set of basic modifications conserving the tree property. We consider in this framework the following basic modifications.

1. add edge action An edge $e=(u, v) \in E(g) \backslash E(t r)$ can be added to $t r$ if $t r$ is empty, or if there is exactly one node $u$ or $v$ in the tree $t r: u \in V(t r)$ XOR $v \in V(t r)$. This edge is called an insertable edge. The insertion of this edge implicitly adds its endpoints to $t r$ if they do not exist in $t r$. The set of insertable edges of $t r$ is denoted by Inst $(t r)$ and this insertion action is denoted by $a d d E d g e(t r, e)$. We also use $a d d E d g e(t r, e)$ to denote the resulting tree. The first basic neighborhood is the following:

$$
N T_{1}(t r)=\{\operatorname{addEdge}(\operatorname{tr}, e) \mid e \in \operatorname{Inst}(\operatorname{tr})\}
$$

2. remove edge action An edge $e=(u, v) \in E(t r)$ can be removed from $t r$ if one node $u$ or $v$ is a leaf of $t r: \operatorname{deg}_{t r}(u)=1 \vee \operatorname{deg}_{t r}(v)=1$. This edge is called a $r e$ movable edge. The removal of this edge thus also removes its endpoints if they are the leaves of $t r$. The set of removable edges of $t r$ is denoted by $\operatorname{Remv}(t r)$ and this removal action is denoted by removeEdge $(t r, e)$. We also use removeEdge $(t r, e)$ to denote the resulting tree. The second basic neighborhood is defined as follows:

$$
N T_{2}(t r)=\{\text { removeEdge }(\operatorname{tr}, e) \mid e \in \operatorname{Remv}(\operatorname{tr})\}
$$

3. replace cycle edge action [2] An edge $e^{\prime}$ of $t r$ can be replaced by another edge $e=(u, v) \in E(g) \backslash E(t r)$ with $u, v \in V(t r)$ conserving the tree property in the following case: the insertion of $e$ creates a fundamental cycle containing $e^{\prime}$ and the removal of $e^{\prime}$ removes the cycle and restores the tree property. The edge $e$ is called a replacing edge, and $e^{\prime}$ is called a replaceable edge of $e$. The set of 
nodes of $t r$ is unchanged by this replacement. We denote by $\operatorname{Repl}(t r)$ the set of replacing edges of $t r$ and $\operatorname{Repl}(t r, e)$ the set of replaceable edges of the replacing edge $e$. We use replaceEdge $\left(t r, e^{\prime}, e\right)$ to denote both the replacement action and the resulting tree. The third basic neighborhood is defined as follows:

$$
N T_{3}(t r)=\left\{\text { replaceEdge }\left(t r, e^{\prime}, e\right) \mid e \in \operatorname{Repl}(t r) \wedge e^{\prime} \in \operatorname{Repl}(\operatorname{tr}, e)\right\}
$$

In practice, we can combine the above basic moves to perform more complex moves. For instance, we take $a d d E d g e\left(t r, e_{1}\right)$ and removeEdge $\left(t r, e_{2}\right)$ at hand where $e_{1} \in \operatorname{Remov}(t r)$ and $e_{2} \in \operatorname{Inst}(t r)$ and $e_{1}$ and $e_{2}$ do not have common endpoint that is the leaf $t r^{2}$. The set of such pairs of $\left\langle e_{1}, e_{2}\right\rangle$ is denoted by RemvInst $(t r)$. This kind of neighborhood has been considered in the tabu search algorithm of [20]. The formal definition of this neighborhood is

$$
N T_{1+2}(t r)=\left\{\operatorname{addEdge}\left(\text { removeEdge }\left(t r, e_{2}\right), e_{1}\right) \mid\left\langle e_{1}, e_{2}\right\rangle \in \operatorname{RemvInst}(t r)\right\}
$$

In the following section, we introduce a novel neighborhood for COP applications.

\subsection{COP neighborhood}

We consider in this paper only elementary paths, i.e., paths having no repeated vertices. These are those which appear in most COP applications. Our constructed framework also supports the modeling of paths where vertices or edges can be repeated, but this will not be presented here (see more details in [53]).

For COP problems, a neighborhood of a path defines a set of paths that can be reached from the current path. The most general neighborhood of a path $p$ on a given graph $g$ is defined as the set of paths generated by replacing a subpath of the current path by another path on the given graph conserving the path property: $\mathcal{N}(p)=\{\operatorname{repl}(p, q) \mid q \in \mathfrak{R}(p)\}$ in which $\mathfrak{R}(p)$ is the set of paths $q$ satisfying followings conditions:

- $q \in g$

- $s(q), t(q) \in p$

- $s p_{p}(s(q)) \cap q=\{s(q)\}$

- $\quad t p_{p}(t(q)) \cap q=\{t(q)\}$

Conditions (3) and (4) ensure the path property of all elements of $\mathcal{N}(p)$ (no repeated vertices are allowed in a path except starting and terminating vertices $)^{3}$.

Unfortunately, such a neighborhood is too large and does not allow being explored in a generic way. To overcome this difficulty, in this section, we propose a restricted neighborhood based on rooted spanning trees. This notion can be widely applied and allows users to perform efficient neighborhood explorations.

2 This condition ensures the preservation of the tree property under the modification action.

3 By some authors, walks with no repeated vertices are referred to as elementary paths. 
Related work As far as we know, there exist only a few local search approaches for COP applications on general graphs. Moreover, these local search algorithms do not explicitly describe neighborhood structures. Rather, the authors talk about the moves, which are very specific and sophisticated. Such moves do not enable the compositionality, modularity, and reuse of the local search programs.

On complete graphs, some local search algorithms have been applied for solving the traveling salesman problem [41] or the vehicle routing problem [34], [9]. In these approaches, a path is explicitly represented by a sequence of vertices and the neighborhood consists of paths generated by changing some vertices of this sequence (e.g., by removing, inserting, exchanging, or changing the position of some vertices). These neighborhood structures cannot be applied to general graphs because a sequence of vertices can not be guaranteed to always form a path on the given graph.

To obtain a reasonable efficiency, a local search algorithm must maintain incremental data structures that allow a fast exploration of this neighborhood and a fast evaluation of the impact of the moves (differentiation). The key novel contribution of our COP framework is to use a rooted spanning tree to represent the current solution and its neighborhood. It is based on the observation that, given a spanning tree $t r$ whose root is $t$, the path from a given node $s$ to $t$ in $t r$ is unique. Moreover, the spanning tree implicitly specifies a set of paths that can be reached from the induced path and provides a data structure for evaluating their desirability. The rest of this section describes the neighborhood in detail. Our COP framework considers both directed and undirected graphs, but, to simplify the presentation, only undirected graphs are treated.

\subsubsection{Rooted spanning trees}

Given an undirected graph $g$ and a target node $t \in V(g)$, our COP neighborhood maintains a spanning tree of $g$ rooted at $t$. Moreover, since we are interested in elementary paths between a source $s$ and a target $t$, the data structure also maintains the source node $s$ and is called a rooted spanning tree (RST) over $(g, s, t)$. An RST $t r$ over $(g, s, t)$ specifies a unique path from $s$ to $t$ in $g$ : path $h_{t r}(s)=\left\langle v_{1}, v_{2}, \ldots, v_{k}\right\rangle$ in which $s=v_{1}, t=v_{k}$ and $v_{i+1}=f a_{t r}\left(v_{i}\right), \forall i=1, \ldots, k-1$. By maintaining RSTs for COP problems, our framework avoids an explicit representation of the paths and enables the definition of a connected neighborhood that can be explored efficiently. Indeed, the tree structure directly captures the path structure from a node $s$ to the root; simple updates to the RST (e.g., an edge replacement) will induce a new path from $s$ to the root. In this framework, we also consider COP applications in which the sources and the destinations of the paths are not fixed. Hence, the source $s$ and the destination (or root) of the RST $(g, s, t)$ can also be changed (but this will not be presented in this paper, interested readers can refer to the $\mathrm{PhD}$ thesis [53]).

Given an RST $t r$ over $(g, s, t)$, we denote by path $(t r)$ the path path $h_{t r}(s)$ which is the path induced by $t r$ from $s$ to the root $t$ of $t r$. Given an undirected graph $g$ and a path $p$ on $g$, we denote by RSTInduce $(g, p)$ the set of RSTs of $g$, rooted at $t(p)$, which induce $p$.

We define in the following section the neighborhood structure based on edge replacements. In COP applications, generally, a candidate solution is a set of paths. 
Each path has its own neighborhood. A neighborhood of a candidate solution is the set of candidate solutions generated by changing some paths of the current candidate solution with their neighbors. Hence, we present only neighborhoods of one path.

\subsubsection{The edge-replacement based neighborhood}

We first show in this section how to update an RST $\operatorname{tr}$ over $(g, s, t)$ based on edge replacements to generate a new rooted spanning tree $t r^{\prime}$ over $(g, s, t)$ which induces a new path from $s$ to $t$ in $g: p^{p t} h_{t r^{\prime}}(s) \neq \operatorname{path}_{t r}(s)$.

Let $t r$ be an RST over $(g, s, t)$, we consider the third basic neighborhood of $t r$ (see Section 3.1):

$$
N T_{3}(t r)=\left\{\text { replaceEdge }\left(t r, e^{\prime}, e\right) \mid e \in \operatorname{Repl}(t r) \wedge e^{\prime} \in \operatorname{Repl}(\operatorname{tr}, e)\right\}
$$

which is the set of RST of $(g, s, t)$. It is easy to observe that two RSTs $t r_{1}$ and $t r_{2}$ over $(g, s, t)$ may induce the same path from $s$ to $t$. For this reason, we now show how to compute a subset $E R N P_{1}(t r) \subseteq N T_{3}(t r)$ such that $\operatorname{path}_{t r^{\prime}}(s) \neq$ path $_{t r}(s), \forall t r^{\prime} \in$ $\operatorname{ERNP}_{1}(t r)$.

We first fix some notations to be used in the following presentation. Given an RST $t r$ over $(g, s, t)$ and a replacing edge $e=(u, v)$, the nearest common ancestors of $s$ and the two endpoints $u, v$ of $e$ are both located on the path from $s$ to $t$. We denote by $\operatorname{lownca}_{t r}(e, s)$ and upnca $_{t r}(e, s)$ the nearest common ancestors of $s$ on the one hand and one of the two endpoints of $e$ on the other hand, with the condition that upnca $_{t r}(e, s)$ dominates $\operatorname{lownca}_{t r}(e, s)$. We denote by $l_{o w}(e, s)$, $u p_{t r}(e, s)$ the endpoints of $e$ such that $n c a_{t r}\left(s, \operatorname{low}_{t r}(e, s)\right)=\operatorname{lownca}_{t r}(e, s)$ and $n c a_{t r}\left(s, u p_{t r}(e, s)\right)=u_{p n c a_{t r}}(e, s)$. Figure 2 illustrates these concepts. The left part of the figure depicts the graph $g$ and the right side depicts an RST tr over $(g, s, r)$. Edge $(8,10)$ is a replacing edge of $t r ; n c a_{t r}(s, 10)=12$ since 12 is the common ancestor of $s$ and 10. $n c a_{t r}(s, 8)=7$ since 7 is the common ancestor of $s$ and 8. lownca $_{t r}((8,10), s)=7$ and upnca $_{t r}((8,10), s)=12$ because 12 Dom $_{t r} 7$; $\operatorname{low}_{t r}((8,10), s)=8 ; \operatorname{up}_{t r}((8,10), s)=10$.

We now specify the replacements that induce a new path from $s$ to $t$.

Proposition 1 Let tr be an RST over $(g, s, t), e=(u, v)$ be a replacing edge of $t r$, let $e^{\prime}$ be a replaceable edge of $e$, and let $t r^{\prime}=\operatorname{rep}\left(t r, e^{\prime}, e\right)$. Let $s u=u p n c a_{t r}(e, s)$ and $s v=$ lownca $_{t r}(e, s)$. We have that path $_{t r^{\prime}}(s) \neq \operatorname{path}_{t r}(s)$ if and only if

(1) $s u \neq s v$ and

(2) $e^{\prime} \in$ path $_{t r}(s v, s u)$

A replacing edge $e$ of $t r$ satisfying the condition (1) is called a preferred replacing edge and a replaceable edge $e^{\prime}$ of $e$ in $t r$ satisfying condition (2) is called a preferred replaceable edge of $e$. We denote by $\operatorname{prefRepl}(t r)$ the set of preferred replacing edges of $t r$ and by $\operatorname{prefRepl}(t r, e)$ the set of preferred replaceable edges of the preferred replacing edge $e$ on $t r$. We also denote by $\operatorname{rep}\left(t r, e^{\prime}, e\right)$ the action and the resulting RST of replacing a preferred replaceable edge $e^{\prime}$ by a preferred replacing edge $e$ on 

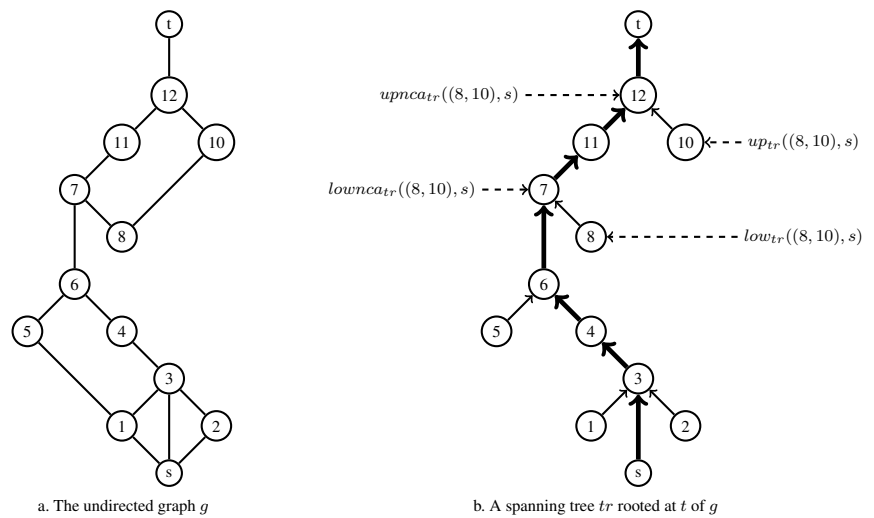

Fig. 2 An Example of Rooted Spanning Tree
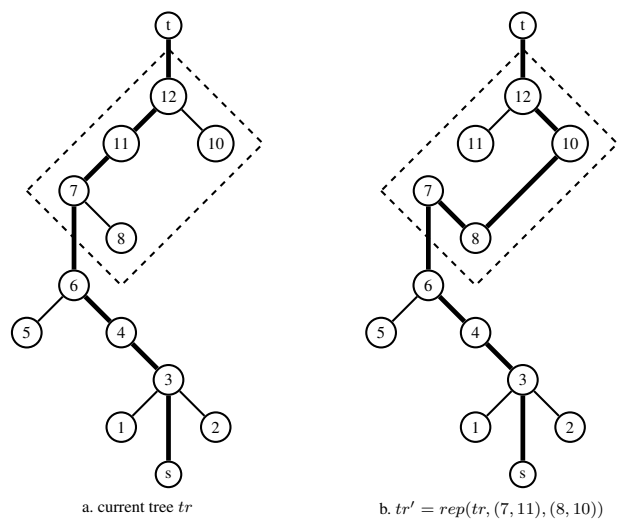

Fig. 3 Illustrating a Basic Move

the RST $t r$. The edge-replacement based neighborhood (called ER-neighborhood) of an RST $t r$ is defined by

$$
\operatorname{ERNP}_{1}(t r)=\left\{t r^{\prime}=\operatorname{rep}\left(t r, e^{\prime}, e\right) \mid e \in \operatorname{prefRepl}(t r), e^{\prime} \in \operatorname{prefRepl}(t r, e)\right\} .
$$

The action $\operatorname{rep}\left(t r, e^{\prime}, e\right)$ is called an ER-move and is illustrated in Figure 3. In the current tree $\operatorname{tr}$ (see Figure $3 \mathrm{a}$ ), the edge $(8,10)$ is a preferred replacing edge, $n c a_{t r}(s, 8)=$ 7, nca $_{t r}(s, 10)=12$, lownca $_{t r}((8,10), s)=7$, upnca $_{t r}((8,10), s)=12$, low $_{t r}((8,-$ $10), s)=8$ and $u p_{t r}((8,10), s)=10$. The edges $(7,11)$ and $(11,12)$ are preferred replaceable edges of $(8,10)$ because these edges belong to path ${ }_{t r}(7,12)$. The path induced by $\operatorname{tr}$ is $\langle s, 3,4,6,7,11,12, t\rangle$. The path induced by $t r^{\prime}$ is $\langle s, 3,4,6,7,8$, $10,12, t\rangle$ (see Figure 3b).

ER-moves ensure that the neighborhood is connected, which is explained in detail in Proposition 2.

Proposition 2 Let $t r^{0}$ be an RST over $(g, s, t)$ and $\mathcal{P}$ be a path from $s$ to $t$. An RST inducing $\mathcal{P}$ can be reached from $t^{0}$ in $k \leq l$ basic moves, where $l$ is the length of $\mathcal{P}$. 


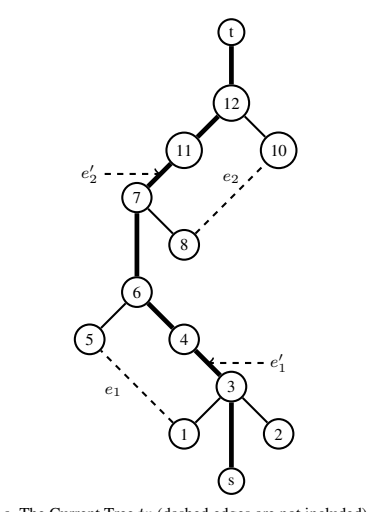

Fig. 4 Illustrating a Complex Move

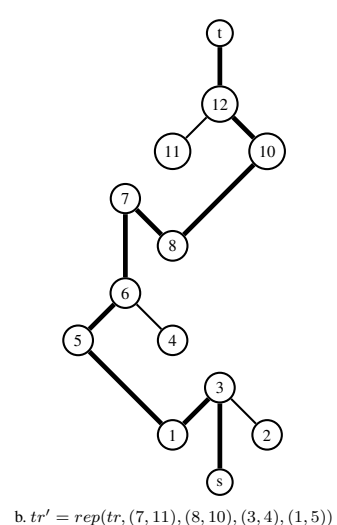

\subsubsection{Neighborhood of independent ER-moves}

It is possible to consider more complex moves by applying a set of independent ERmoves. Two ER-moves are independent if the execution of the first one does not affect the second one and vice versa. The sequence of ER-moves $\left\langle\operatorname{rep}\left(\operatorname{tr}, e_{1}^{\prime}, e_{1}\right), \ldots, r e p(-\right.$ $\left.\left.\operatorname{tr}, e_{k}^{\prime}, e_{k}\right)\right\rangle$, denoted by $r e p\left(t r, e_{1}^{\prime}, e_{1}, e_{2}^{\prime}, e_{2}, \ldots, e_{k}^{\prime}, e_{k}\right)$, is defined as the application of the sequence of actions $\left\langle\operatorname{rep}\left(\operatorname{tr}_{1}, e_{1}^{\prime}, e_{1}\right), \operatorname{rep}\left(\operatorname{tr}_{2}, e_{2}^{\prime}, e_{2}\right), \ldots, \operatorname{rep}\left(\operatorname{tr}_{k}, e_{k}^{\prime}, e_{k}\right)\right\rangle$, where $t r_{1}=t r$ and $t r_{j+1}=\operatorname{rep}\left(t r_{j}, e_{j}^{\prime}, e_{j}\right), \forall j=1, \ldots, k-1$. It is feasible if the ER-moves are feasible, i.e., $e_{j} \in \operatorname{prefRpl}\left(\operatorname{tr}_{j}\right)$ and $e_{j}^{\prime} \in \operatorname{prefRpl}\left(t r_{j}, e_{j}\right)$.

Proposition 3 Consider $k$ ER-moves $r e p\left(t r, e_{1}^{\prime}, e_{1}\right), \ldots, r e p\left(t r, e_{k}^{\prime}, e_{k}\right)$. If all possible execution sequences of these basic moves are feasible and the edges $e_{1}^{\prime}, e_{1}, e_{2}^{\prime}, e_{2}$,$\ldots, e_{k}^{\prime}, e_{k}$ are all different, then these $k$ ER-moves are independent.

We denote by $E R N P_{k}(t r)$ the set of neighbors of $t r$ obtained by applying $k$ independent ER-moves. The action of taking a neighbor in $E R N P_{k}(t r)$ is called an ER- $k$ move.

It remains to find some criterion for whether two ER-moves are independent. Given an RST $\operatorname{tr}$ over $(g, s, t)$ and two preferred replacing edges $e_{1}, e_{2}$, we say that $e_{1}$ dominates $e_{2}$ in tr, written $e_{1}$ Dom $_{t r} e_{2}$, if lownca lo $_{t r}\left(e_{1}, s\right)$ dominates upnca $a_{t r}\left(e_{2}, s\right)$. Then, two preferred replacing edges $e_{1}$ and $e_{2}$ are independent w.r.t. $t r$ if $e_{1}$ dominates $e_{2}$ in $t r$ or $e_{2}$ dominates $e_{1}$ in $t r$.

Proposition 4 Let tr be an RST over $(g, s, t), e_{1}$ and $e_{2}$ be two preferred replacing edges such that $e_{2} \operatorname{Dom}_{t r} e_{1}, e_{1}^{\prime} \in \operatorname{prefRpl}\left(\operatorname{tr}, e_{1}\right)$, and $e_{2}^{\prime} \in \operatorname{prefRpl}\left(\operatorname{tr}, e_{2}\right)$. Then rep $\left(t r, e_{1}^{\prime}, e_{1}\right)$ and $\operatorname{rep}\left(t r, e_{2}^{\prime}, e_{2}\right)$ are independent and the path induced by rep $\left(t r, e_{1}^{\prime}, e_{1}, e_{2}^{\prime}, e_{2}\right)$ is path par $_{\text {t }}\left(s, v_{1}\right)+$ path $_{t r}\left(u_{1}, v_{2}\right)+$ path $_{t r}\left(u_{2}, t\right)$, where the addition sign denotes path concatenation and $v_{1}=\operatorname{low}_{t r}\left(e_{1}, s\right), u_{1}=u p_{t r}\left(e_{1}, s\right)$, $v_{2}=\operatorname{low}_{t r}\left(e_{2}, s\right)$, and $u_{2}=u p_{t r}\left(e_{2}, s\right)$.

Figure 4 illustrates a complex move. In $t r$, the two preferred replacing edges $e_{1}=$ $(1,5)$ and $e_{2}=(8,10)$ are independent because lownca $a_{t r}((8,10), s)=7$, which dominates upnca $_{t r}((1,5), s)=6$ in $t r$. The new path induced by $t r^{\prime}$ is $\langle s, 3,1,5,6$, $7,8,10,12, t\rangle$, which is actually the path $\operatorname{path}_{t r}(s, 1)+\operatorname{path}_{t r}(5,8)+$ path $_{t r}(10, t)$. 


\section{Data Structure and Algorithms}

In this section, we briefly describe the implementation of some fundamental and nontrivial abstractions and then analyze their complexities.

\subsection{VarTree and nearest common ancestors}

VarTree $(g)$ is an abstraction representing a dynamic tree over an undirected graph $g$ that can be modified by removing, inserting an edge, or replacing an edge by another edge. It also allows querying information about the tree. For facilitating manipulations on dynamic trees, the trees are implicitly stored as rooted trees. Several well-known data structures have been proposed for representing dynamic trees, for instance, ST-trees [57,58], topology trees [33], ET-trees [36], top trees [6,59], and RC-trees [1] (and the references therein). These data structures maintain a forest of dynamic rooted trees, supporting update actions (e.g., link and cut) and some queries (e.g., minimum (maximum) cost edge, node on a path, nearest common ancestors of two nodes, medians, centers of a tree) in $\mathcal{O}(\log n)$ time per operation where $n$ is the number of vertices of the given graph. These data structures have been experimentally studied in [60]. These data structures are dedicated to implementing specific network algorithms, for instance the maximum flow problem.

In the LS (Graph) framework, it is required to maintain a dynamic rooted tree supporting update actions (i.e., add, remove, replace edges) and different basic queries such as nearest common ancestors of two nodes, the father of a node, the set of nodes, edges, the set of adjacent edges of a given node. At each step of the local search process, the system explores a neighborhood, queries the quality of all neighbors, and chooses one neighbor to move. Usually, the neighborhood is large and the neighborhood exploration should be as quick as possible. This exploration requires frequent performances of the above queries over dynamic rooted trees. Queries over dynamic trees should thus be as fast as possible. For this purpose, we use a direct data structure for the tree by maintaining the father of each node, the sets for storing nodes, and the edges and the adjacent edges of each node of the tree. So the time complexity for each update action is $\mathcal{O}(n)$ and the above queries (except for that for the nearest common ancestors) take $\mathcal{O}(1)$ instead of $\mathcal{O}(\log n)$.

Concerning the nearest common ancestors problem, Bender et al. [16] presented a simple optimal algorithm for trees which is a sequentialized version of the more complicated PRAM algorithm of Berkman and Vishkin [17]. An intermediate data structure is precomputed in $\mathcal{O}(n)$; each query $n c a(u, v)$ is then computed in $\mathcal{O}(1)$ time. The data structure is based on Euler Tour and the data structure for the range minimum query (RMQ) problem. We apply the data structure of [16] with an incremental implementation. This means we partially update the data structure whenever the tree is modified (i.e., by adding, removing, or replacing edges) instead of recomputing it from scratch. This incremental implementation does not improve the time complexity in the worst case $(\mathcal{O}(n)$ for each update action) but it is more efficient in practice. We have tested this implementation on dynamic trees of size 98, 198, 498, 998, of complete graphs of size 100, 200, 500, 1000. For each graph, we generate 
randomly 20 sequences of 10,000 update actions (adding, removing, replacing edges) conserving the size of the tree. The experimental results show that this incremental implementation is about 1.6 times faster than recomputing from scratch.

\subsection{Maintaining weighted distances between vertices on dynamic trees}

NodeDistances $(v t)$ is a graph invariant which maintains the weighted distances between all pairs of vertices of a VarTree $v t$. This invariant allows querying the cost of the path between any pair of nodes in $\mathcal{O}(1)$, and thus allows querying the differentiations in $\mathcal{O}(1)$ in some cases, for instance, querying the change in the cost of a path under edge replacement actions. To implement this graph invariant, we use a direct 2-dimensional data structure $\operatorname{dis}$ : $\operatorname{dis}(u, v)$ represents the cost of the path from $u$ to $v$ on the current RST $t r$. The size of this data structure is $\mathcal{O}\left(n^{2}\right)$ but at any time of computation, it is maintained and used partially: only those $\operatorname{dis}(u, v)$ such that $v$ dominates $u$ on the current tree $t r$ are considered.

The cost of any two nodes $x$ and $y$ on $t r$ can be queried by Algorithm 1 in $\mathcal{O}(1)$ where line 1 can be queried in $\mathcal{O}(1)$.

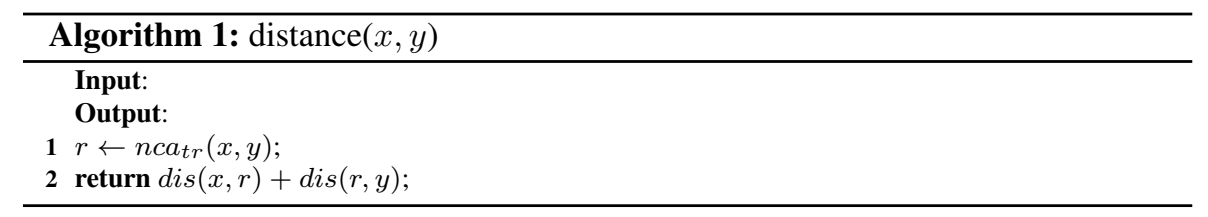

We now show how to update the $\operatorname{dis}(x, y)$ data structure under a local move on $t r$, viz., $r e p\left(t r,\left(u_{1}, v_{1}\right),\left(u_{2}, v_{2}\right)\right)$. Without loss of generality, suppose that $v_{1}$ Dom $_{t r} v_{2}$ and $u_{1}$ Dom $_{t r} v_{1}$ (see an example in Figure 5). We put $S=\left\{x \in V(t r) \mid v_{1} D_{o m_{t r}}\right.$ $x\}$. The following elements of the data structure should be updated: $\operatorname{dis}(x, y), \forall x \in$ $S, y \in$ path $_{t r}\left(v_{2}, n c a_{t r}\left(x, v_{2}\right)\right) \cup$ path $_{t r}\left(u_{2}\right)$. The update schema is given in Algorithm 2 , in which $c\left(u_{2}, v_{2}\right)$ is the weighted distance between $u_{2}$ and $v_{2}$ in the given graph (see line 6).

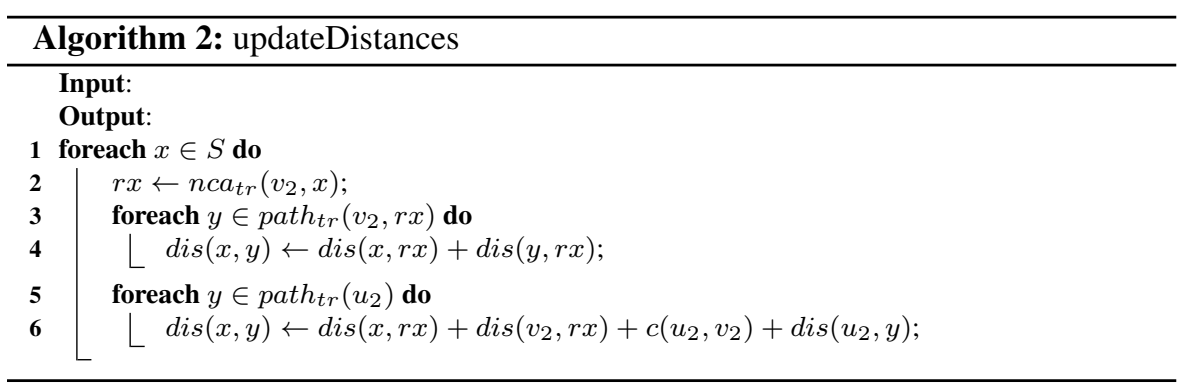




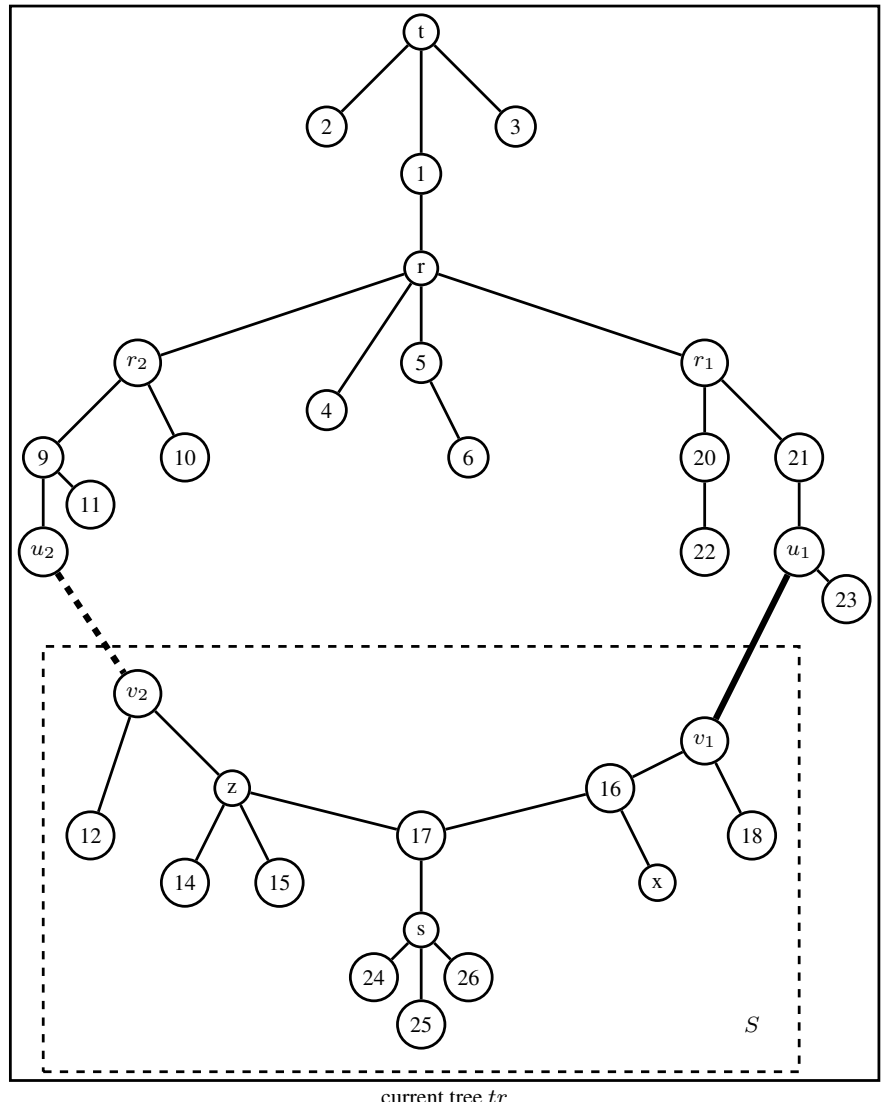

Fig. 5 Ilustrating the update of $\operatorname{dis}(u, v)$ under the replaceEdge $(t r,(u 1, v 1),(u 2, v 2))$ action

The worst case time complexity is $\mathcal{O}\left(n^{2}\right)$ but it performs more efficiently in practice. We now experimentally analyze the efficiency of incrementality in comparison with recomputation from scratch. To do so, we analyze the ratio $r_{i}=\frac{s_{i-1}}{S_{i}}$ of data structures to be updated (i.e., $\operatorname{dis}(u, v)$ ) where $S_{i}$ is the number of elements of dis to be maintained at each step $i$ of the computation:

$$
S_{i}=\sum_{v \in V\left(t r^{i}\right)} c_{t r^{i}}(v)
$$

where $t r^{i}$ is the tree at step $i$ and $c_{t r^{i}}(v)$ is the number of nodes on the path from $v$ to the root of $t r^{i} ; s_{i}$ is the number of elements of dis to be changed at step $i$ by the incremental version. We look at dynamic trees of size 98, 198, 498, 998 on complete graphs of size $100,200,500,1000$. For each graph, we randomly generate 20 sequences of 10,000 moves. The experimental results show that the average value of $r_{i}$ is about $\frac{1}{10}$. Figures 6,7 show the number of elements to be updated and the number of total elements to be maintained in the last 20 iterations: each iteration is a replace edge action or a sequence of 2 actions (add and remove edge). It is clear that in the remove edge action, we do not need to update the data structures, so the number of elements to be updated in this action is zero. 


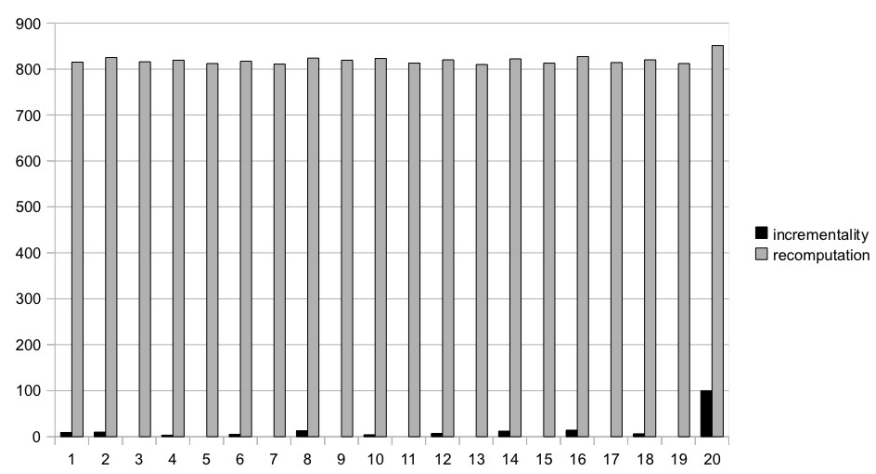

Fig. 620 last iterations for a complete graph of size 100

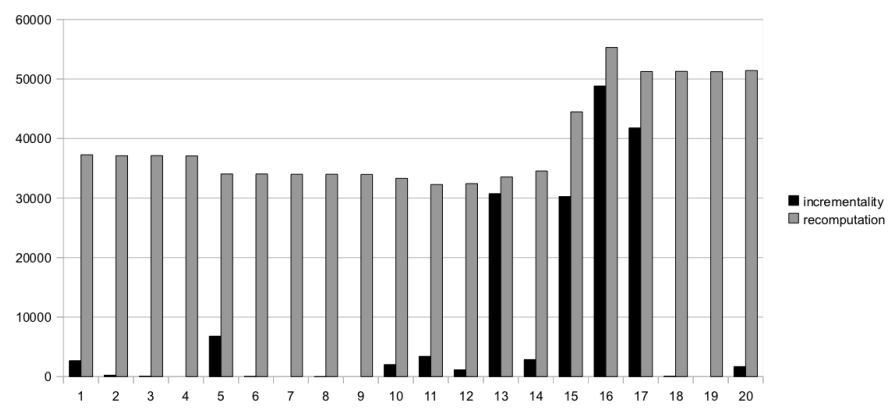

Fig. 720 last iterations for a complete graph of size 1000

\section{Implementation in COMET}

The LS (Graph) framework is implemented in COMET [62]. That is an extension (about 25,000 lines of COMET code) of the COMET system. The core of the framework is the graph variables (e.g., VarTree, VarPath objects representing dynamic trees, paths which can be changed) over which are defined the graph invariants, graph constraints, and graph functions. The graph invariants maintain the properties of dynamic trees and paths such as the set of insertable, removable, or replacing edges of a VarTree, the sum of weights of all the edges of a path, and the diameter of a tree. The graph constraints and graph functions are differentiable objects which not only maintain the properties of dynamic trees, paths (for instance, the number of violations of a constraint or the value of an objective function), but also allow determining the impact of local moves on these properties, a feature known as differentiation. 


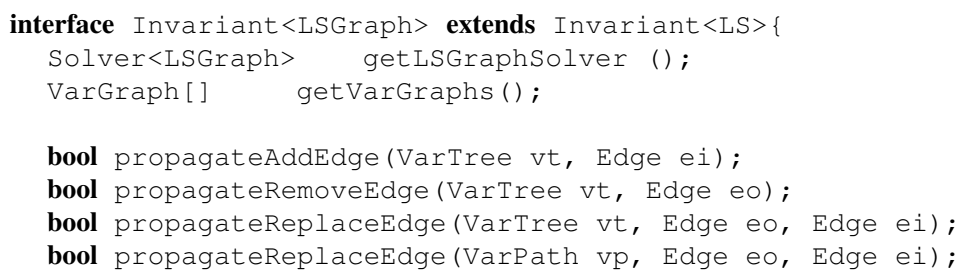

Fig. 8 interface of graph invariants (partial description)

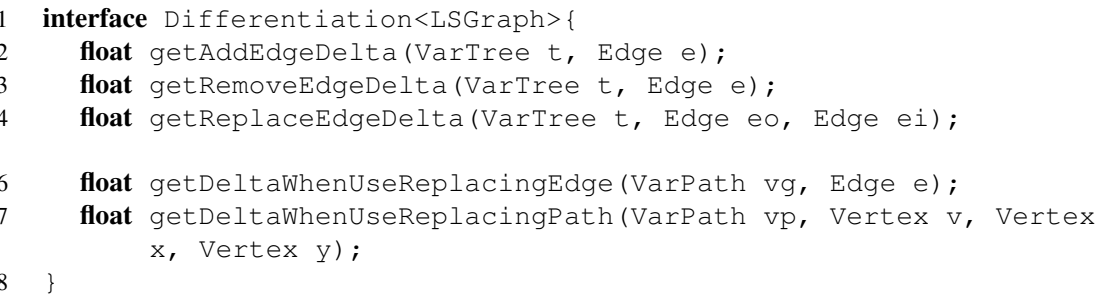

Fig. 9 differentiation interface (partial description)

\subsection{Interfaces}

Figure 8 depicts part of the interface concerning the graph invariants. Line 2 returns a Solver<LSGraph> object which manages all graph variables and graph invariants, and maintains a precedence graph relating these graph variables and graph invariants of the model. A local move (modification action) over a graph variable (VarTree, VarPath) induces a propagation which updates all graph invariants, constraints, and functions that are defined over these variables thanks to the precedence graph. This means that one does not have to call procedures to update graph invariants, constraints, or functions. Rather, the update is automatically performed whenever users apply local moves. Line 3 returns the list of graph variables ${ }^{4}$ over which the graph invariant is defined. Lines 5-8 are some propagation methods corresponding to different local moves.

The differentiation interface is depicted in Figure 9. The differentiation methods evaluate the impact of various local moves, for instance, getAddEdgeDelta(VarTree vt, Edge e) in line 2 computes the change in the value of the function when the edge $e$ is added to the tree $v t$; the method in line 6 returns the change in the value of the function when the replacing edge $e$ is applied ${ }^{5}$. The method in line 7 is generic and computes the impact of moves where the subpath of vp between two endpoints of $x$ and $y$ is replaced by the path $\langle x, v, y\rangle$ (see the definition of the

4 VarGraph is an abstract class from which VarTree, VarPath are derived.

5 When a local move replace $E d g e\left(t r, e^{\prime}, e\right)$ is applied with the neighborhood $E R N P_{1}$ (see Section 3.2 ), the resulting path depends only on the replacing edge $e$ used, not on the replaceable edge $e^{\prime}$. 


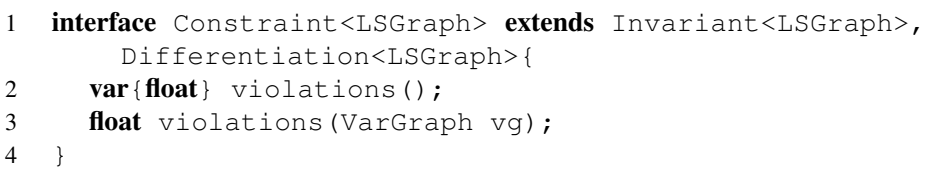

Fig. 10 interface of graph constraints (partial description)

most general COP neighborhood $\mathcal{N}$ at the beginning of Section 3.2). It enables the exploration of neighborhoods other than the $E R N P_{1}$.

Figure 10 depicts the interface of graph constraints in which the method in line 2 returns the violations of the constraint. Line 3 returns the violations of the constraint attributed to VarGraph vg. If the graph variable does not appear directly in the definition of the constraint, it does not contribute any violations. This information may be useful when applying multistage heuristics.

All graph invariants, functions, and constraints in the system must implement these interfaces. This enables the compositionality of model. Moreover, one can design and implement one's own functions and constraints, respecting these interfaces, and integrate them into the system.

\subsection{Abstractions}

The Solver<LS> of COMET does not support specific operations on user-defined objects (i.e., edge replacement on dynamic trees). So in this framework, we designed and implemented a Solver<LSGraph> which maintains a precedence graph representing the dependence of graph invariants, graph functions, and graph constraints on the graph variables and performs the propagations for updating the graph invariants, graph functions, and graph constraints under different modification actions over the graph variables. The implementation of Solver $<\mathrm{LSGraph}>$ extends Solver $<\mathrm{LS}>$, enabling combinations between the two solvers (e.g., we can combine standard invariants of COMET with graph invariants of LS (Graph) by arithmetic operators). Table 1 partially presents some abstractions ${ }^{6}$ available in the framework including some graph variables, invariants, functions, and constraints which are used to model various COT/COP problems: create a solver Solver $<\mathrm{LSGraph}>$, declare variables VarTree, VarPath, and state functions and constraints. Different search procedures can then be performed over the model. Fundamental functions representing relations between the trees, paths, nodes, and edges have been designed and implemented, e.g., NBVisitedVerticesTree (VarTree [] vts, set\{Vertex\} $S$ ) represents the number of vertices of $S$ which are visited by the list of trees vts, and NBVisitsVertexTree (VarTree[] vts, Vertex v) represents the number of times the list of trees vts visit it. Weight (VarTree vt, int k) represents the weight of a tree $\mathrm{vt}$, and PathCostonEdges (VarPath vp, int $\mathrm{k}$ ) represents the cost of a path $\mathrm{vp}^{7}$. These functions can be combined by traditional

\footnotetext{
6 For a full description of the abstractions, see the PhD thesis [53].

$7 \mathrm{k}$ is the index of the considered weight on edges.
} 

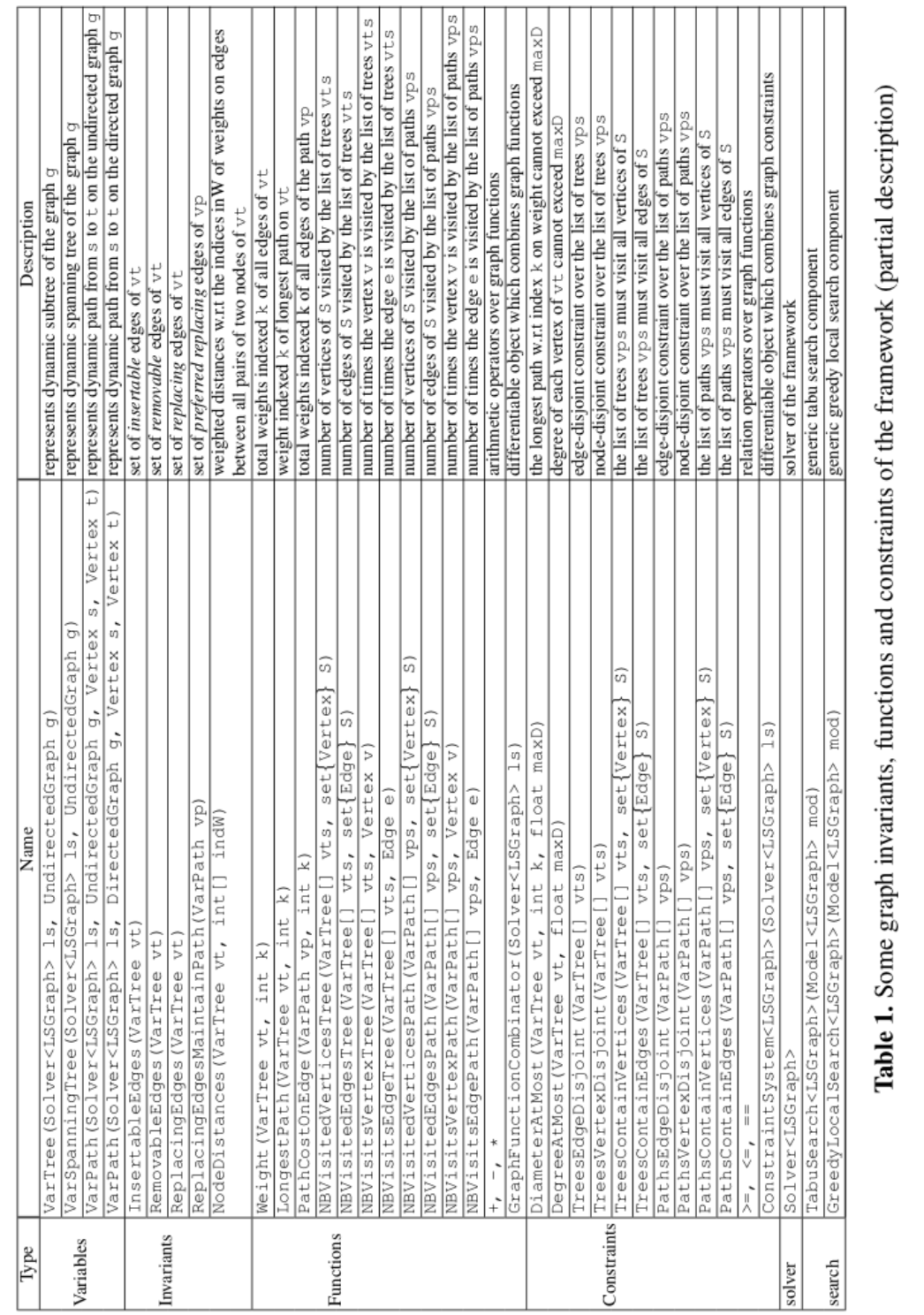

arithmetic or relation operators to state more complex functions or constraints. Various fundamental constraints on graphs can be stated by using these functions and traditional relation operators. For achieving a more efficient performance, some global constraints have been designed and implemented, for instance, PathsEdgeDis joint (VarPath [ ] Vps) specifies that the list of paths vps must be edge-disjoint, and PathsContainVertices (VarPath [] vps, set $\{$ Vertex\} S) specifies that the list of paths vps must visit the set of vertices $S$. 
FunctionCombinator<LSGraph $>$ is a graph function that combines several functions, constraints of the model by the "+" operator with a weight. This object strengthens the modeling of the framework when there are a number of functions proportional to the size of the problem to be stated.

ConstraintSystem<LSGraph> is a graph constraint which combines all constraints appearing in the considered problem by the post method. By using this object, one can add or remove some constraints from the model without having to change the search procedure.

The LS (Graph) framework is open in that it allows users to design and implement their own invariants, constraints, and functions respecting predefined interfaces and integrate them into the system.

\subsection{Search procedures}

In order to illustrate the modeling and the search component, we give an example in Figure 11 in which we solve the problem of finding a spanning tree of a given undirected graph $g$ such that the degree of each node does not exceed maxDe and the diameter of the spanning tree does not exceed maxDia.

The model is given in lines $1-15$, in which line 2 creates a Solver<LSGraph> Is and lines 3-4 randomly initialize a spanning tree variable vt of a given undirected graph $g$ associated with $1 \mathrm{~s}$. Line 5 initializes a graph invariant $\mathrm{rpl}$ (line 4) representing the set of replacing edges of vt. Lines 7-13 state and post constraints on the degree and diameter of the spanning tree $v t$ to a graph constraint system gCs which is declared in line 10. Whenever the model is closed (line 15), the initPropagation methods of all graph invariants are called to initialize the values and internal data structures of these objects.

The search is given in lines $17-26$, which is a simple greedy search. At each iteration, we explore the $N T_{3}$ neighborhood and choose the best neighbor w.r.t. the graph constraint system gcs: we choose a replacing edge ei and a replaceable edge eo of ei such that the number of violations of gCs is most reduced when eo is replaced by ei (see method getReplaceEdgeDelta (vt, eo, ei)). Line 23 is the local move which induces automatically a propagation to update all graph invariants and constraints defined over it (e.g., rpl, degreeC, diameterC) thanks to the precedence graph maintained in $1 \mathrm{~s}$.

We can see in this example that the model and the search are independent. On the one hand, we can state and post other constraints to the graph constraint system ges without having to change the search. On the other hand, we can apply different heuristic local searches in the search component without changing the model.

We now describe one of generic neighborhood explorations. Figure 12 explore the basic COP neighborhood $E R N P_{1}$. The quality of a solution is evaluated in terms of the number of violations of the Constraint<LSGraph> c. Variables it and fgb represent the current iteration of the local search and the smallest value of the number of violations of the constraint $c$ found so far. All VarPath vps [j] are scanned (lines 7-8). Line 9 retrieves the Invariant<LSGraph> repl representing the 


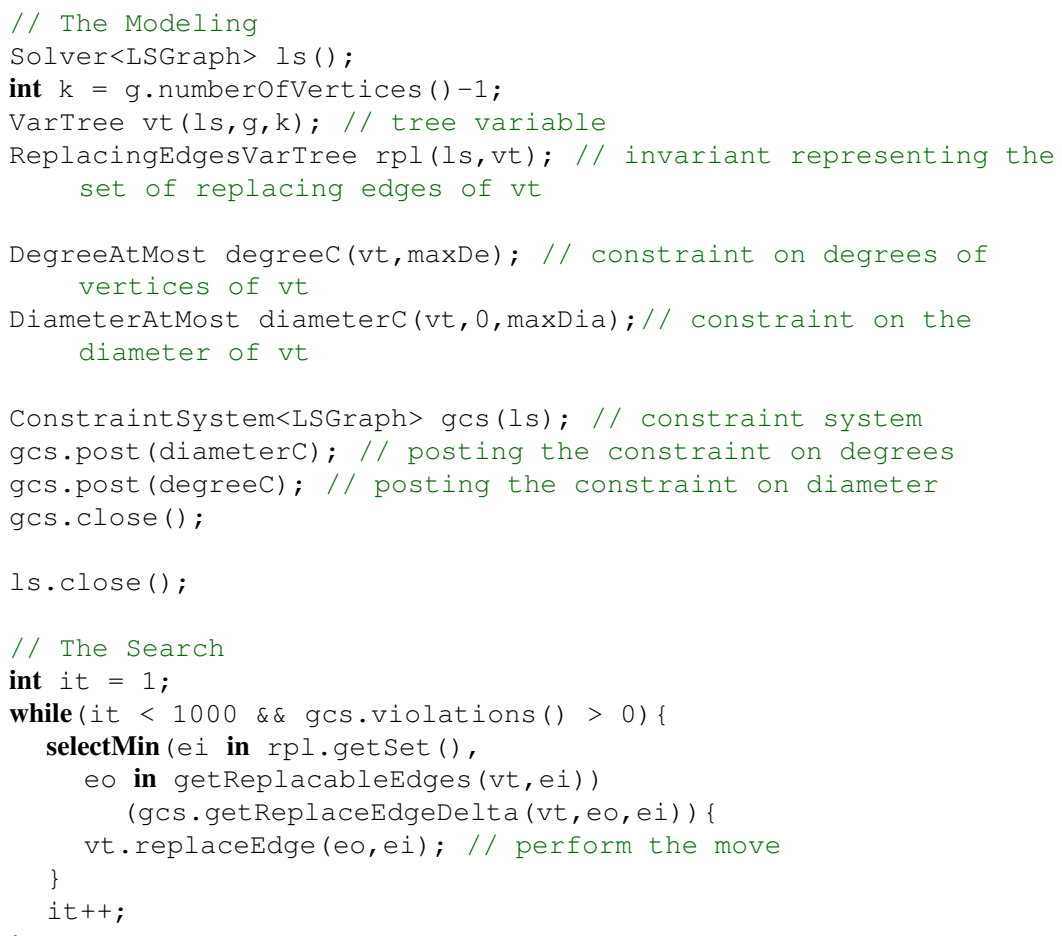

Fig. 11 Model for bounded diameter and degree constrained spanning tree

set of preferred replacing edges of $\operatorname{vps}[j]$. All preferred replacing edges e are scanned in line 10 and line 11 evaluates the quality of the move when applying the replacing edge $e$ in term of the variation of the number of violations of $c$. Line 13 checks whether $e$ is tabu or the aspiration criterion is reached (i.e., the move is tabu but it improves the best solution found so far). Lines 31-33 choose a preferred replaceable sel_eo. Lines 36-41 submit a move (lines 36-41) and its evaluation eval to a Neighborhood $\mathrm{N}$ and it will be called later.

Components for a generic tabu search, TabuSearch $<\mathrm{LSGraph}>$, and a greedy local search, GreedyLocal Search<LSGraph>, have been implemented for COT/COP applications. This tabu search component features aspiration criteria with adaptive tabu length (the tabu length can be changed within tbMin and tbMax, depending on the behavior of the search). A full description of the abstractions and generic search components can be found in [53].

\section{Applications}

In this section, we present the application of the LS (Graph) framework to the resolution of three COT/COP problems: the quorumcast routing $(\mathrm{QR})$ problem, the edge- 
1

void exploreTabuMinReplace1Move1VarPath(Neighborhood N, VarPath[] vps, dict $\{$ VarPath->ReplacingEdgesMaintainPath\} mapReVarPath, Constraint<LSGraph> c, GTabuEdge[] tbIn, GTabuEdge[] tbout, int it, float fgb, bool firstImprovement) \{

int ind $=-1 ; /$ the index of the selected VarTree for the

float eval = System.getMAXINT (); // the minimum evaluation

forall $(j$ in vps.rng ()$)\{$

VarPath vp = vps[j]; // considered VarPath

ReplacingEdgesMaintainPath repl = mapReVarPath $\{v p\} ; / /$ invariant representing the set of preferred replacing edges of vp

forall (e in repl.getset()) \{ // scan all preferred replacing edges

float $d=c$.getDeltaWhenUseReplacingEdge (vp,e); // evaluation of using the preferred replacing edge $e$

if (!tbIn[j].isTabu (e, it) || d + c.violations()< fgb) \{ / / check the tabu condition or the aspiration criterion if $(d<$ eval $)\{/ /$ update the information of the chosen move eval $=d ;$ ind $=j$; sel_ei $=e$;

if (firstImprovement \&\& eval < 0 ) break; // stop the neighborhood exploration if a first improving neighbor is found \}

\}

if (firstImprovement \&\& eval < 0 )

break; // stop the neighborhood exploration if a first improving neighbor is found

\}

if $($ ind $>-1)$;

Varpath $\mathrm{vp}=\operatorname{vps}[$ ind];

Edge sel_eo = null;

select (eo in getPreferredReplacableEdges (vp, sel_ei)) \{

sel_eo = eo;

if $($ sel_eo $!=$ null $)$ neighbor (eval,N) \{// submit the chosen move

tbIn[ind].makeTabu (sel_eo,it); // make the selected preferred replacable edge tabu

tbout [ind].makeTabu(sel_ei,it); // make the selected preferred replacing edge tabu

vp.replaceEdge (sel_eo, sel_ei); // perform the move

\}

\}

Fig. 12 Exploring the $E R N P_{1}$ neighborhood 
disjoint paths (EDP) problem, and the routing and wavelength assignment with side constraint (RWA-D) problem.

For the first and the third applications (QR and RWA-D), we apply tabu search. Two parameters of tabu search are the length $t b l$ of the tabu lists and maxStable: if the best-restart solution ${ }^{8}$ does not improve in maxStable successive local moves, then the search is restarted.

Experiments were performed on XEN virtual machines with 1 core of a CPU Intel Core2 Quad Q6600 @2.40GHz and 1GB of RAM.

\subsection{The quorumcast routing $(\mathrm{QR})$ problem}

\subsubsection{Problem statement}

Given a weighted undirected graph $G=(V, E)$, each edge $e \in E$ is associated with a cost $w(e)$. Given a source node $r \in V$, an integral value $q$, and a set $S \subseteq V$ of multicast nodes, the quorumcast routing problem is to find a minimum cost tree $T=\left(V^{\prime}, E^{\prime}\right)$ of $G$ spanning $r$ and $q$ nodes of $S . T=\left(V^{\prime}, E^{\prime}\right)$ is a graph satisfying

1. $V^{\prime} \subseteq V \wedge E^{\prime} \subseteq E$,

2. $T$ is connected,

3. $\exists Q \subseteq S$ such that $\sharp Q=q \wedge Q \cup\{r\} \subseteq V^{\prime}$,

4. The cost of

$$
T=\sum_{e \in E^{\prime}} w(e)
$$

is minimum over all subgraphs of $G$ with properties 1,2 , and 3 .

In this section, we present a local search model for solving the QR problem with LS (Graph).

\subsubsection{The model}

We propose a tabu search model in LS (Graph) exploring different neighborhoods for solving this problem. The model is given in Figure 13, in which line 1 creates a Solver LLSGraph $>$ and line 2 declares a VarTree $t r$ associated with 1 s. Lines 4-7 state the constraints of the problem where NBVisitedVertices (tr, S) is a Function<LSGraph> representing the number of vertices of $S$ which are in the tree $t r$. The constraint posted in line 5 says that the tree $t r$ must contain at least $q$ vertices of $S$ and the constraint posted in line 6 says that $t r$ must contain the vertex $s$. Line 9 creates a Model<LSGraph> mod with only one variable $t r$, the constraint gcs, the objective function to be minimized is the total weight of tr. Line 11 initializes a search component which extends TabuSearch $<\mathrm{LSGraph}>$ (see Figure 14). Lines 12-14 set parameters for the search and line 16 calls the search procedure. We now describe the search component in Figure 14. The variables_card and _root represent the number of edges of the initial tree and its root computed in the

\footnotetext{
8 The best-restart solution is the best solution found for each restart.
} 


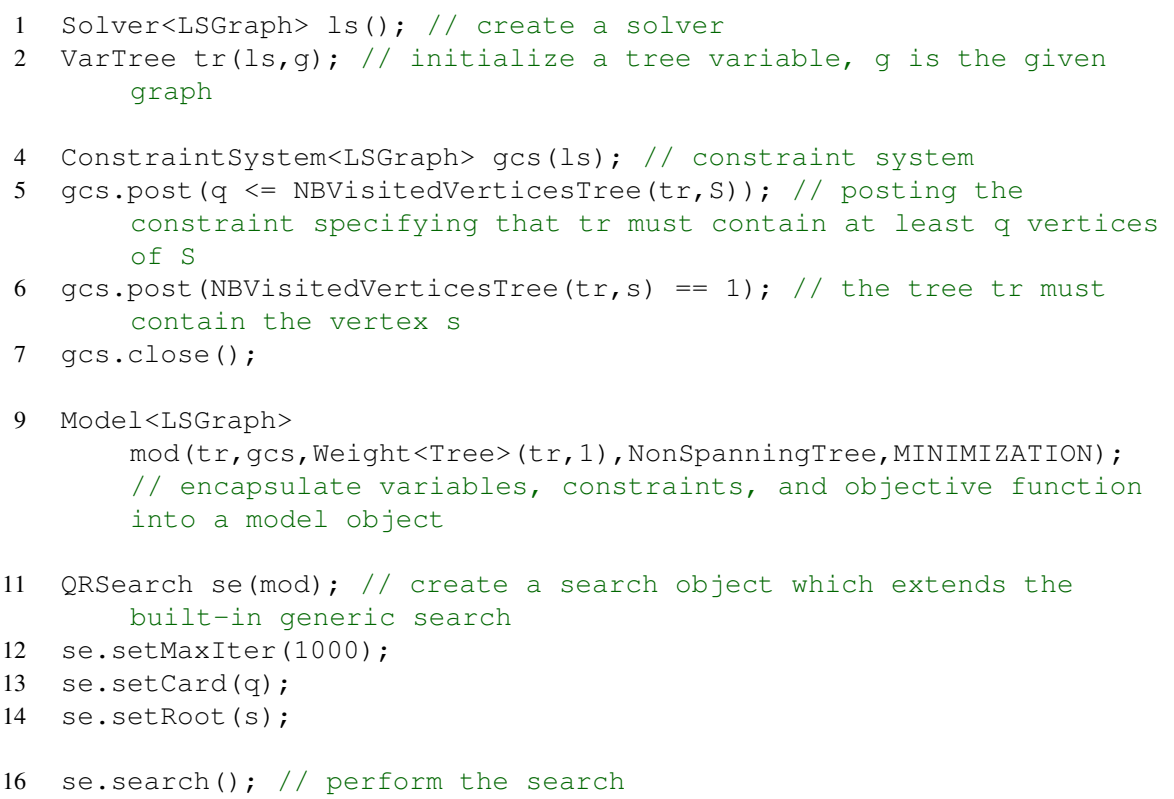

Fig. 13 Tabu search model for the QR problem

initSolution method. The overriding initSolution method (lines 17-31) constructs the tree in a greedy random way. It clears the tree $\operatorname{tr}$ (line 22) and selects randomly a first edge containing _root (lines 23-25). It then iteratively selects an edge with minimal weight for adding to the constructed tree tr (lines 27-30). The exploreNeighborhood method of TabuSearch<LSGraph $>$ is also overriden (lines 34-39) with different neighborhoods: $N T_{1}$ (line 35), $N T_{2}$ (line 36), $N T_{1+2}$ (line 37), and $N T_{3}$ (line 38).

\subsubsection{Experiments}

We compare our tabu model in LS (Graph) with the IMP heuristic, which is the best heuristic among the three heuristic algorithms in [24]. The original instances and the implementation of the IMP algorithm are not available. We thus re-implemented the IMP algorithm in COMET and generated new benchmarks.

Problem instances We take six graphs from the benchmark of the KCT problem [20] which are 4-regular graphs of sizes from 50 to 1000 nodes and six graphs from the Steiner tree instances. For each graph of size $n$, we generate randomly $n * \operatorname{tau}_{1}$ nodes for the set $S$, the value for $q$ is set to $n * t a u_{1} * t a u_{2}$ with $\operatorname{tau}_{1}, \operatorname{tau}_{2} \in\{0.2,0.5\}$, and the root is set to be node 1 .

Results The IMP algorithm and our model in LS (Graph) are executed 20 times for each problem instance. The time limit for our model is 30 minutes. From our pre- 
1 include "LS(Graph)";

class QRSearch extends TabuSearch $<$ LSGraph $>\{$

Vertex root:

int_card;

QRSearch (Model<LSGraph> mod) : TabuSearch<LSGraph> (mod) \{

\}

void setCard (int ca) \{

\}

card $=\mathrm{ca}$

void setRoot (Vertex $r)\{$

root $=r$;

void restartSolution() \{ // restart the search by using the initial solution generation procedure

initsolution();

void initSolution() $\{/ /$ generate the initial solution

Solver<LSGraph> Is = getLSGraphSolver(); // get the solver

VarTree $\operatorname{tr}=$ getFirstVarTree (); // retrieve the tree variable tr

InsertableEdgesVarTree inst = getInsertableEdges(tr); //

retrieve the invariant representing the set of insertable edge of tr

tr.clear(); // clear the tree

select (e in inst.getset ():e.contains (root) ) \{ // choose randomly a first edge to be added to tr

tr.addEdge (e); \}

forall ( $i$ in 1.._card-1) // repeat adding an edge until the tree tr has _card edges

selectMin (e in inst.getSet ()) (e.weight()) \{ // select an insertable edge having smallest weight

tr.addEdge (e); // add the selected edge to the tree

29

30

31 \}

void exploreNeighborhood(Neighborhood N) \{ // explore all four neighborhoods of VarTree

exploreTabuMinAdd1VarTree (N, true); // explore the neighborhood NT_1

exploreTabuMinRemove1VarTree (N, true); // explore the neighborhood NT_2

37 exploreTabuMinAddRemove1VarTree (N,true); // explore the neighborhood NT_\{1+2\}

38 exploreTabuMinReplacelVarTree (N,true); // explore the neighborhood NT_3 \}

\}

Fig. 14 The search component for the QR problem 


\begin{tabular}{|c|c|c|c|c|c|c|c|c|c|c|c|}
\hline \multirow{2}{*}{ Index } & \multirow{2}{*}{ Instances } & \multicolumn{5}{|c|}{ IMP } & \multicolumn{5}{|c|}{ LS(Graph) } \\
\hline & & avg & $\min$ & $\max$ & std_dev & avg_t & avg & $\min$ & $\max$ & std_dev & avg_t \\
\hline 1 & g50_20_20 & 111 & 111 & 111 & 0 & 0.78 & 111 & 111 & 111 & 0 & 0.06 \\
\hline 2 & g50_20_50 & 251 & 251 & 251 & 0 & 0.78 & 248 & 248 & 248 & 0 & 0.08 \\
\hline 3 & g50_50_20 & 169 & 169 & 169 & 0 & 0.8 & 169 & 169 & 169 & 0 & 0.09 \\
\hline 4 & g50_50_50 & 386 & 386 & 386 & 0 & 0.76 & 369 & 369 & 369 & 0 & 0.22 \\
\hline 5 & g75_20_20 & 93 & 93 & 93 & 0 & 1.06 & 93 & 93 & 93 & 0 & 0.02 \\
\hline 6 & g75_20_50 & 358 & 358 & 358 & 0 & 1.06 & 328 & 328 & 328 & 0 & 1.12 \\
\hline 7 & g75_50_20 & 207 & 207 & 207 & 0 & 1.05 & 175 & 175 & 175 & 0 & 0.08 \\
\hline 8 & g75_50_50 & 630 & 630 & 630 & 0 & 1.05 & 564.6 & 560 & 568 & 3.56 & 181.81 \\
\hline 9 & g100_20_20 & 178 & 178 & 178 & 0 & 1.44 & 178 & 178 & 178 & 0 & 0.23 \\
\hline 10 & g100_20_50 & 526 & 526 & 526 & 0 & 1.45 & 524 & 524 & 524 & 0 & 2.31 \\
\hline 11 & g100_50_20 & 294 & 294 & 294 & 0 & 1.51 & 273 & 273 & 273 & 0 & 0.28 \\
\hline 12 & g100_50_50 & 948 & 948 & 948 & 0 & 1.53 & 854 & 854 & 854 & 0 & 36.84 \\
\hline 13 & g200_20_20 & 428 & 428 & 428 & 0 & 7.13 & 402 & 402 & 402 & 0 & 32.6 \\
\hline 14 & g200_20_50 & 926 & 926 & 926 & 0 & 7.07 & 849.6 & 849 & 851 & 0.92 & 342.49 \\
\hline 15 & g200_50_20 & 483 & 483 & 483 & 0 & 7.26 & 468 & 468 & 468 & 0 & 8.09 \\
\hline 16 & g200_50_50 & 1499 & 1499 & 1499 & 0 & 7.35 & 1411.45 & 1403 & 1424 & 5.82 & 816.43 \\
\hline 17 & g400_20_20 & 599 & 599 & 599 & 0 & 52.18 & 556.6 & 551 & 560 & 1.88 & 240.46 \\
\hline 18 & g400_20_50 & 1724.05 & 1702 & 1739 & 13.92 & 51.43 & 1610.95 & 1600 & 1626 & 7.05 & 689.33 \\
\hline 19 & g400_50_20 & 1154.55 & 1140 & 1166 & 7.75 & 51.6 & 1010.65 & 1005 & 1018 & 4.3 & 584.08 \\
\hline 20 & g400_50_50 & 3040 & 3040 & 3040 & 0 & 52.19 & 2829.15 & 2799 & 2856 & 17.25 & 845.52 \\
\hline 21 & g1000_20_20 & 1832.1 & 1810 & 1836 & 9.28 & 812.61 & 1568.65 & 1505 & 1621 & 27.67 & 584.75 \\
\hline 22 & g1000_20_50 & 4762.2 & 4755 & 4771 & 7.96 & 795.64 & 4493.55 & 4406 & 4599 & 49.9 & 869.09 \\
\hline 23 & g1000_50_20 & 2743 & 2733 & 2746 & 4.29 & 801.06 & 2487.2 & 2429 & 2533 & 27.04 & 697.85 \\
\hline 24 & g1000_50_50 & 7293.85 & 7229 & 7361 & 36.28 & 817.82 & 7098.95 & 6891 & 7372 & 117.06 & 1330.21 \\
\hline
\end{tabular}

Table 1 Experimental results on KCT instances

liminary results, we set $t b l$ to 5 and maxStable to 200 . The experimental results are shown in Tables 1 and 2. Columns 3-6 present the average, the minimal, the maximal, and the standard deviation of the best objective value found in 20 executions. The same information for our model is presented in columns 8-11. Column 7 is the average execution time (in seconds) of the IMP algorithm over 20 executions, while column 12 presents the average time (in seconds) for finding the best solutions over 20 executions of our tabu search model. Table 1 shows that for KCT instances, our LS (Graph) model finds better solutions than the IMP on average. Moreover, the worst solutions found by our model are, in most cases, even better than the best solution found by the IMP (among 20 executions). Table 2 shows that the results found by our model are better than those found by the IMP algorithm on average except for the last four instances (45-48). A comparison of the two algorithms in terms of box-and-whiskers plots (see their template presentation in Figure 15) can be found in Figures 16, 17, 18, and 19. Two consecutive bars present the results computed by the IMP and the tabu search algorithms on a given instance. The figures show that for each algorithm, the variance of the results among the 20 executions is small. It also shows that, in most instances, the solutions found by our tabu search are better than those found by the IMP algorithm. 


\begin{tabular}{|l|l|r|r|r|r|r|r|r|r|r|r|}
\hline \multirow{2}{*}{ Index } & \multirow{2}{*}{ Instances } & \multicolumn{9}{|c|}{ IMP } & \multicolumn{10}{c|}{ LS(Graph) } \\
\cline { 3 - 10 } & & avg & min & max & std_dev & avg_t & avg & min & max & std_dev & avg_t \\
25 & steinb4_20_20 & 11 & 11 & 11 & 0 & 0.72 & 11 & 11 & 11 & 0 & 0 \\
26 & steinb4_20_50 & 32 & 32 & 32 & 0 & 0.75 & 32 & 32 & 32 & 0 & 0.12 \\
27 & steinb4_50_20 & 20.35 & 20 & 21 & 0.48 & 0.74 & 20 & 20 & 20 & 0 & 0.08 \\
28 & steinb4_50_50 & 52.25 & 51 & 53 & 0.77 & 0.74 & 41 & 41 & 41 & 0 & 0.27 \\
29 & steinb10_20_20 & 19 & 19 & 19 & 0 & 1 & 19 & 19 & 19 & 0 & 0.12 \\
30 & steinb10_20_50 & 29 & 29 & 29 & 0 & 0.98 & 29 & 29 & 29 & 0 & 0.32 \\
31 & steinb10_50_20 & 27.8 & 26 & 29 & 1.25 & 1.03 & 22 & 22 & 22 & 0 & 0.16 \\
32 & steinb10_50_50 & 65 & 65 & 65 & 0 & 0.99 & 65 & 65 & 65 & 0 & 2.76 \\
33 & steinb16_20_20 & 10 & 10 & 10 & 0 & 1.46 & 10 & 10 & 10 & 0 & 0.01 \\
34 & steinb16_20_50 & 73.35 & 69 & 76 & 2.33 & 1.55 & 61 & 61 & 61 & 0 & 9.46 \\
35 & steinb16_50_20 & 32.85 & 32 & 37 & 1.19 & 1.47 & 31 & 31 & 31 & 0 & 0.93 \\
36 & steinb16_50_50 & 87.25 & 85 & 92 & 2.09 & 1.48 & 82 & 82 & 82 & 0 & 12.6 \\
37 & steinc6_20_20 & 92.35 & 81 & 98 & 4.75 & 100.58 & 69.7 & 69 & 72 & 0.9 & 514.06 \\
38 & steinc6_20_50 & 234.15 & 229 & 240 & 3.61 & 101.77 & 221.9 & 218 & 225 & 1.73 & 614.34 \\
39 & steinc6_50_20 & 130.95 & 122 & 147 & 6.16 & 99.82 & 115.9 & 113 & 118 & 1.09 & 372.27 \\
40 & steinc6_50_50 & 399.55 & 395 & 407 & 3.17 & 103.59 & 381.55 & 374 & 387 & 3.28 & 866.87 \\
41 & steinc11_20_20 & 43.95 & 40 & 47 & 1.99 & 102.08 & 38.7 & 38 & 39 & 0.46 & 481.52 \\
42 & steinc11_20_50 & 116 & 113 & 119 & 1.55 & 102.6 & 107.4 & 107 & 109 & 0.58 & 803.34 \\
43 & steinc11_50_20 & 75.05 & 70 & 79 & 2.48 & 101.89 & 67.75 & 67 & 69 & 0.62 & 455 \\
44 & steinc11_50_50 & 207.25 & 201 & 213 & 2.9 & 102.04 & 202.45 & 199 & 208 & 2.31 & 1000.79 \\
45 & steinc16_20_20 & 22.25 & 21 & 25 & 1.13 & 100.2 & 23.6 & 22 & 24 & 0.66 & 200.7 \\
46 & steinc16_20_50 & 54.45 & 52 & 59 & 1.66 & 100.22 & 54.95 & 53 & 56 & 0.74 & 267.01 \\
47 & steinc16_50_20 & 50 & 50 & 50 & 0 & 99.34 & 50.3 & 50 & 52 & 0.56 & 451 \\
48 & steinc16_50_50 & 125 & 125 & 125 & 0 & 104.98 & 140.25 & 133 & 148 & 4.09 & 1567.46 \\
\hline
\end{tabular}

Table 2 Experimental results on steiner instances

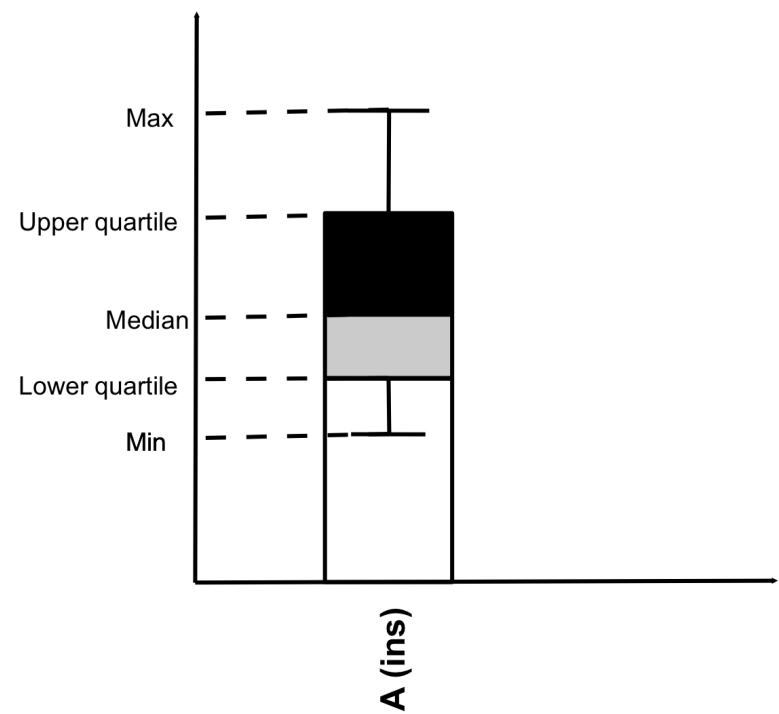

Fig. 15 Box-and-Whiskers plot: The $X$-axis represents the algorithm and the instance (A denotes the algorithm and ins denotes the instance) and the Y-axis represents the value of the objective function 


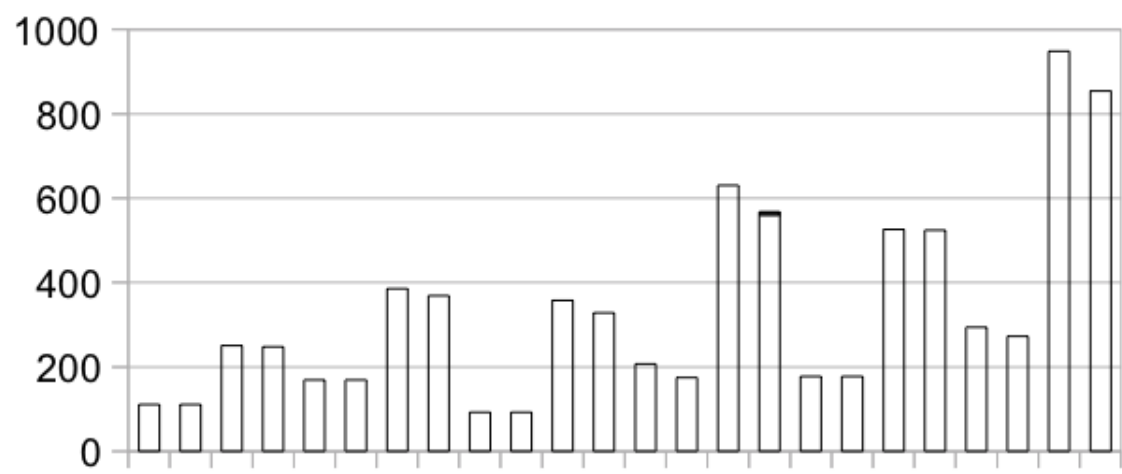

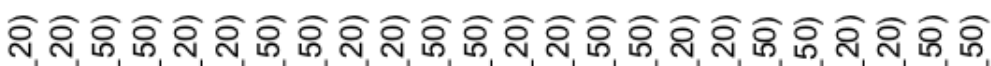

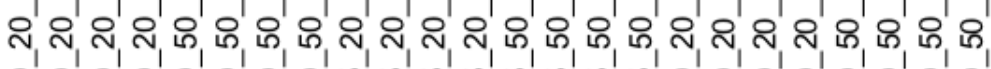

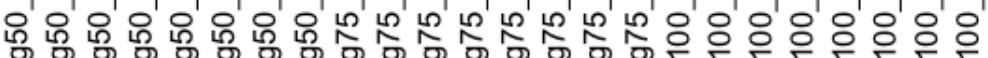

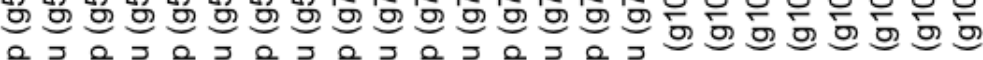

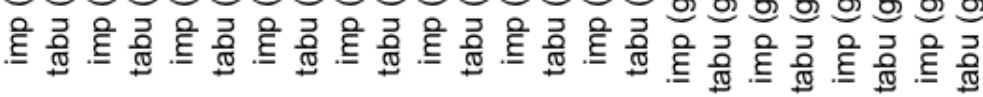

Fig. 16 Comparison between IMP and LS(Graph) on KCT instances

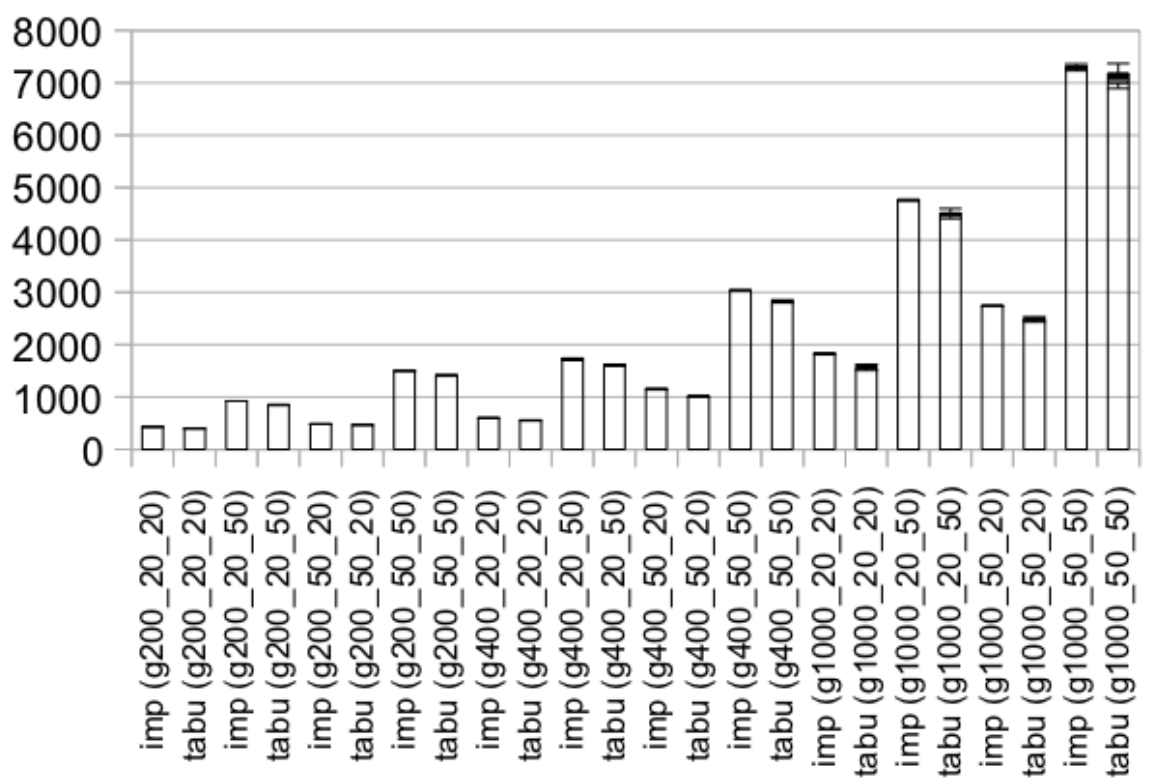

Fig. 17 Comparison between IMP and LS(Graph) on KCT instances 


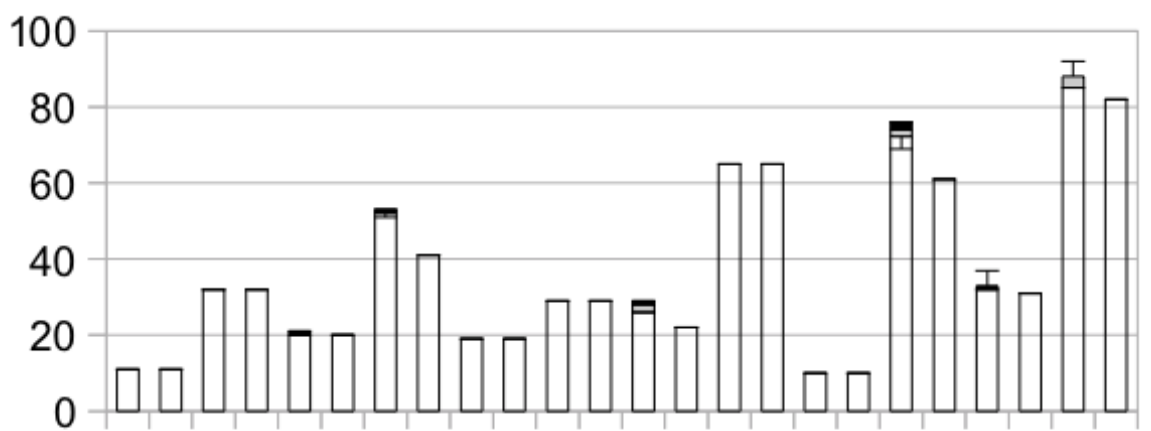

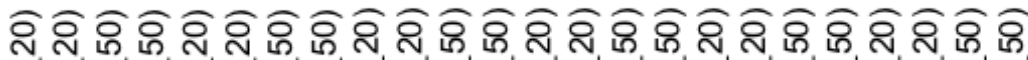

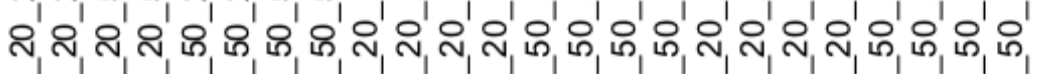
オ

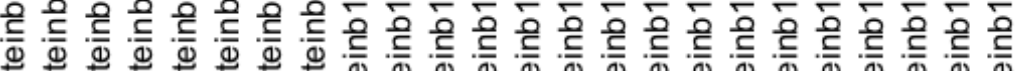

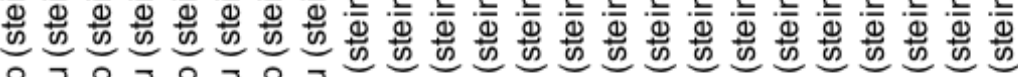

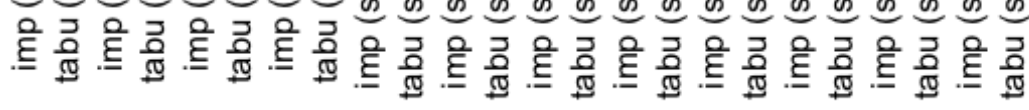

Fig. 18 Comparison between IMP and LS(Graph) on steiner instances

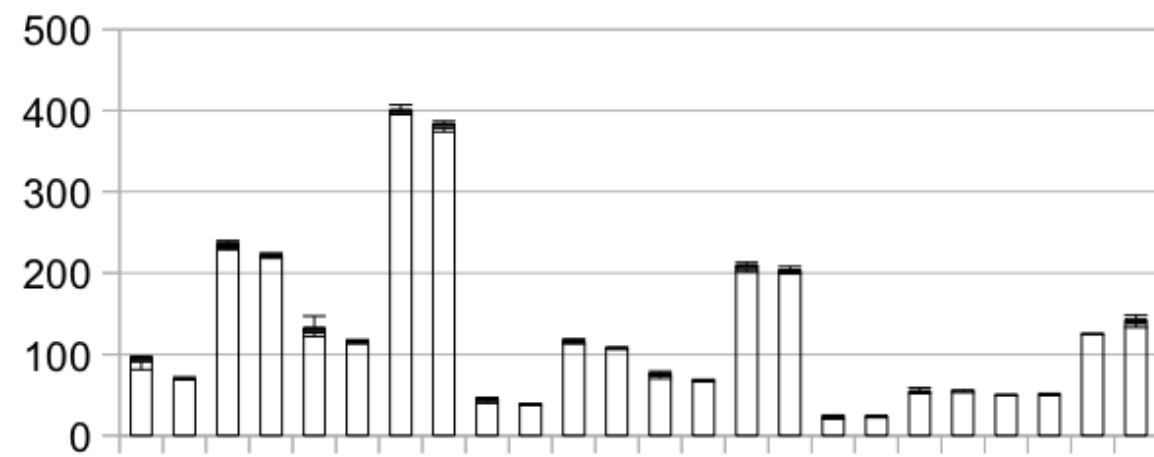

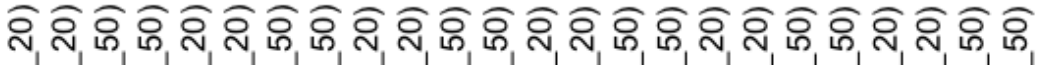
กิ กิ กิ 잉

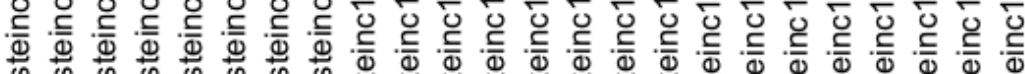

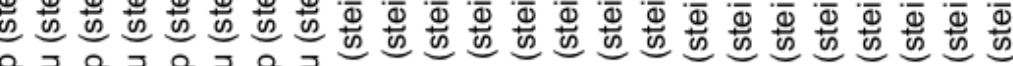

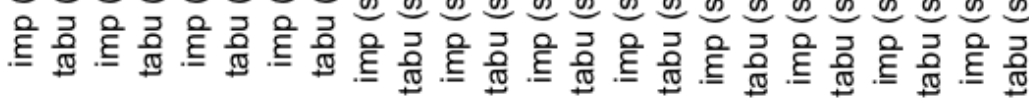

Fig. 19 Comparison between IMP and LS(Graph) on steiner instances 
6.2 The edge-disjoint paths problem

\subsubsection{Problem statement}

We are given an undirected graph $G=(V, E)$ and a set $T=\left\{\left\langle s_{i}, t_{i}\right\rangle \mid i=\right.$ $\left.1,2, \ldots, \sharp T ; s_{i} \neq t_{i} \in V\right\}$ representing a list of commodities. A subset $T^{\prime} \subseteq T$, $T^{\prime}=\left\{\left\langle s_{i_{1}}, t_{i_{1}}\right\rangle, \ldots,\left\langle s_{i_{k}}, t_{i_{k}}\right\rangle\right\}$ is called $e d p$-feasible if there exist mutually edgedisjoint paths from $s_{i_{j}}$ to $t_{i_{j}}$ on $G, \forall j=1,2, \ldots, k$. The EDP problem consists in finding a maximal cardinality $e d p$-feasible subset of $T$. In other words,

$$
\begin{array}{lll}
\max & \sharp T^{\prime} & \text { (1) } \\
\text { s.t. } & T^{\prime} \subseteq T & \text { (2) } \\
& T^{\prime} \text { is edp-feasible } & \text { (3) }
\end{array}
$$

In this section, we propose two algorithms based on neighborhood search for solving the EDP problem by LS (Graph). They are complex heuristics which make use of local search in LS (Graph) as sub-routines. We first describe the simple greedy algorithm SGA [42] because one of our algorithms (detailed later) will apply this as sub-procedure (see Algorithm 3).

\subsubsection{The simple greedy algorithm}

This algorithm starts with an empty solution $S$ (line 1). At each iteration $j$ (line 3), it selects a pair $T_{j}=\left\langle s_{j}, t_{j}\right\rangle$ and tries to find the shortest path $P_{j}$ from $s_{j}$ to $t_{j}$ in the graph $G_{1}=\left(V, E_{1}\right)$, initializing the set of edges $E_{1}$ to be $E$ (line 2). If such a path exists, it is inserted into $S$ and the set $E_{1}$ is updated for the next step by removing all edges of the path $P_{j}$.

Obviously, the SGA algorithm depends strongly on the order of commodities $T_{j}$ considered. The multi-start version of SGA (called MSGA) performs SGA iteratively with different orders of $T_{j}$ to be scanned in $T$.

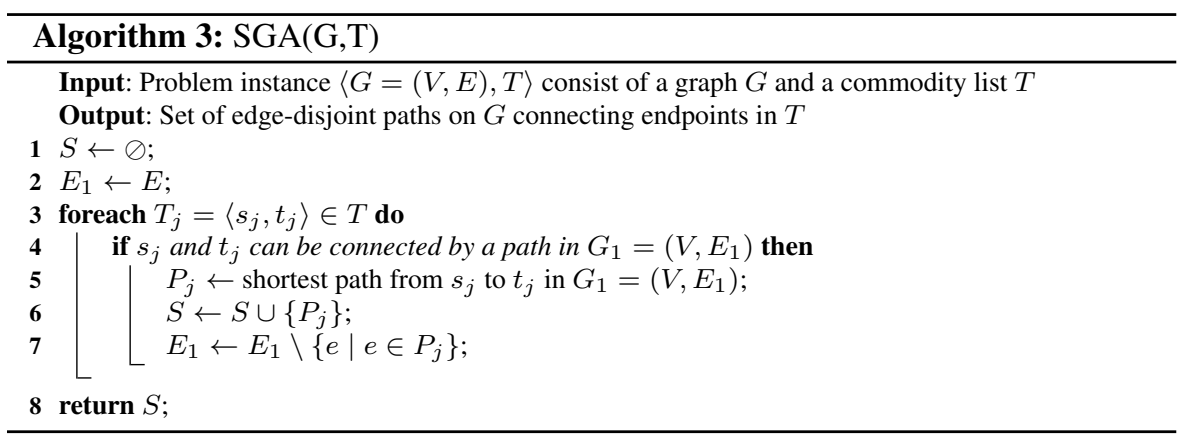

In the ACO algorithm of [18], the following criterion is introduced, which quantifies the degree of non-disjointness of a solution. $S=\left\{P_{1}, P_{2}, \ldots P_{k}\right\}\left(P_{j}\right.$ is a path 
from $s_{j}$ to $\left.t_{j}\right)$ :

$$
C(S)=\sum_{e \in E}\left(\max \left\{0, \sum_{P_{j} \in S} \rho^{j}(S, e)-1\right\}\right),
$$

where $\rho^{j}(S, e)=1$ if $e \in P_{j}$, and $\rho^{j}(S, e)=0$ otherwise. From a solution constructed by ANTs, a solution to the EDP problem is extracted by iteratively removing the path which has the most edges in common with other paths, until all remaining paths are mutually edge-disjoint (see Algorithm 4).

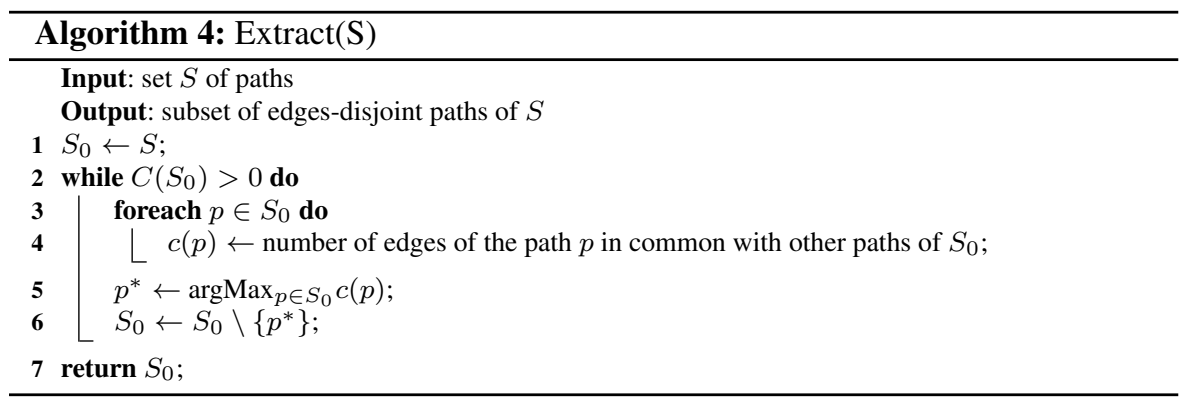

In this section, we propose two algorithms based on local search for solving this problem: the LS-SGA and the LS-R algorithms. These algorithms perform a local search procedure applying the LS (Graph) framework combined with the extraction method (Algorithm 4) and the simple greedy algorithm. These algorithms make use of the PathsEdgeDisjoint $\left(P_{1}, P_{2}, \ldots, P_{k}\right)$ constraint of the LS (Graph) framework saying that the set of paths $\left\{P_{1}, P_{2}, \ldots, P_{k}\right\}$ must be edge-disjoint. The number of violations of the PathsEdgeDisjoint $\left(P_{1}, P_{2}, \ldots, P_{k}\right)$ constraint is defined to be $C\left(\left\{P_{1}, P_{2}, \ldots, P_{k}\right\}\right)$ and the local search algorithms used in our heuristics try to minimize this number.

\subsubsection{The LS-SGA algorithm}

The LS-SGA algorithm has been proposed in our paper [54]. The main idea of the LSSGA algorithm (given in detail in Algorithm 5) is to perform a local search algorithm aiming at minimizing the number of violations of the PathsEdgeDisjoint $\left(P_{1}, P_{2},-\right.$ $\left.\ldots, P_{k}\right)$ constraint. The variable $S$ (line 2 ) stores a set of paths $\left\{P_{1}, P_{2}, \ldots, P_{k}\right\}$ connecting all commodities. It is initialized randomly (lines $3-5$ ). At each step, we perform a local move. The LocalMove method (line 7) returns true if it finds a move that decreases the number of violations of the PathsEdgeDisjoint $\left(P_{1}, P_{2}, \ldots, P_{k}\right)$ constraint. If no such move exists, we make some random moves (line 22). From a candidate solution $S$ found by the local search, a solution $S_{1}$ to the EDP problem will be extracted by applying the Extract algorithm (line 9) combined with the SGA algorithm (line 15) on the remaining graph $G^{\prime \prime}$ (the graph $G^{\prime \prime}$ is obtained by removing all edges $E^{\prime}$ (line 12) of the paths extracted by the Extract algorithm) and the remaining commodities $T^{\prime \prime}$ (lines 10-11). The best solution is updated in line 17 and lines 18-20 update some paths of $S$ by the new found paths of $S_{2}$. 


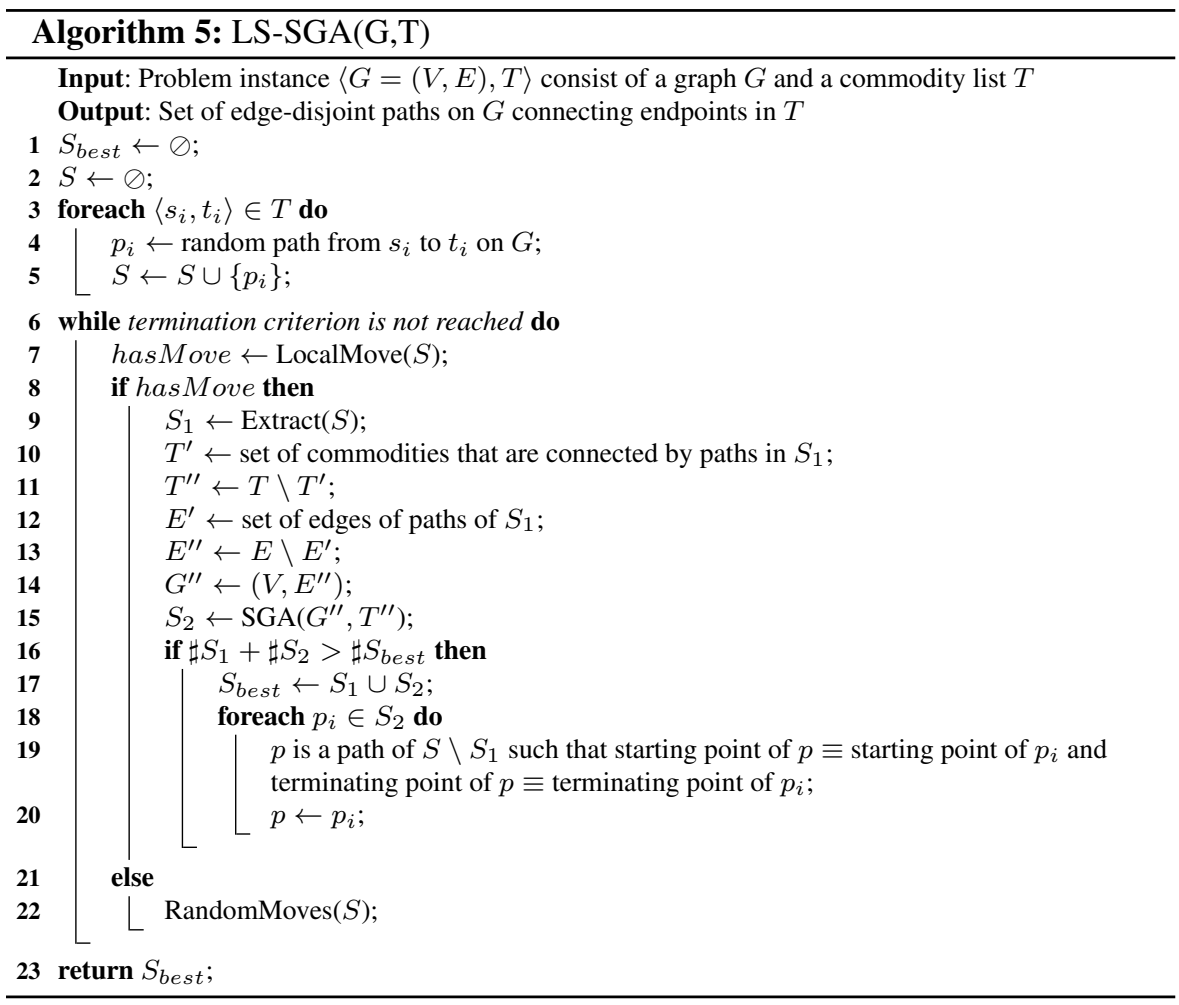

\subsubsection{The LS-R algorithm}

The idea is to connect recursively as much as possible the commodities of $T$ (see Algorithm 6). The core is the recursive method LS-Recursive in Algorithm 7, which receives a graph $G$ and a list of commodities $T$ as input and computes a set of maximally edge-disjoint paths connecting the commodities of $T$. This paths set is then accumulated in the solution $\mathrm{Sol}$ ( $\mathrm{Sol}$ is a global variable) and all edges visited by these paths are removed from $G$ for the next recursive call. Line 1 computes a set of edge-disjoint paths by a greedy local search method, GreedyLocalSearch. Lines 2-3 update the solution by adding the new found edge-disjoint paths of $S_{i}$. Lines 3-4 compute the set of connected components $C C$ of the graph generated from the current graph by removing all edges $E^{\prime}$ of paths of $S_{i}$. For each graph $G_{i}$ of these connected components and each set of commodities $T_{i}$ that belong to $G_{i}$, we perform recursively the LS-Recursive method (see lines 6-8).

The implementation of these algorithms in LS (Graph) is given in the $\mathrm{PhD}$ thesis [53]. It is more complicated than that of the QR problem: it requires some processing (e.g., removing edges and vertices from a graph, and computing the connected components of a graph) other than just stating the model and performing the search. 

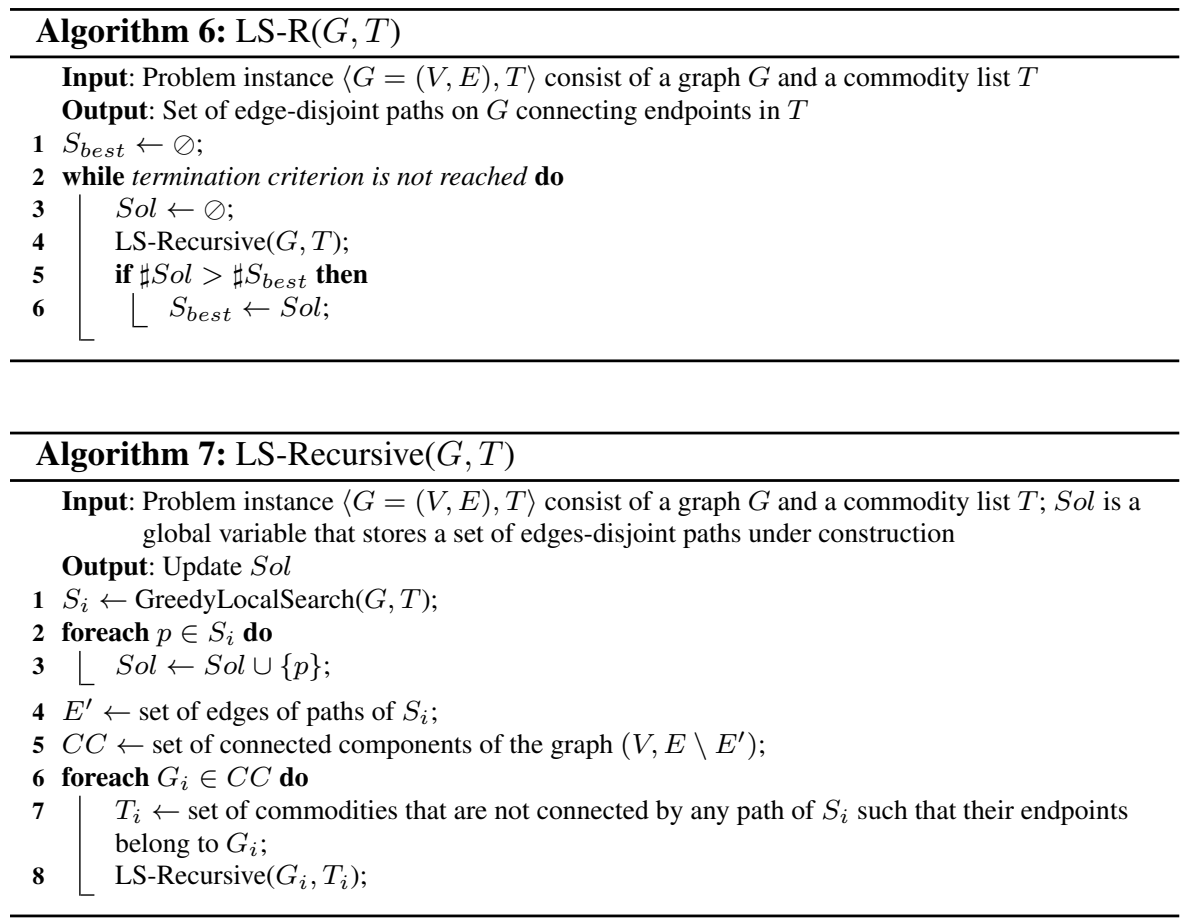

\subsubsection{Experiments}

Problem instances We tried the two proposed algorithms on three types of benchmark. The first benchmark contains instances on four graphs provided by Blesa [18]. The second benchmark contains instances on some graphs of the Steiner benchmark from the Or-Library [14]. The third benchmark consists of instances on random planar graphs. Table 3 gives a description of these graphs.

An instance of the EDP problem consists of a graph and a set of commodities. The instances in the original paper [18] are not available. As a result, we base our trial on the instance generator described in [18] and generate new instances as follows. For each graph of the first set, we generate randomly different sets of commodities with different sizes, depending on the size of the graph: for each graph of size $n$, we generate randomly two instances ${ }^{9}$ with $0.10^{*} n, 0.25^{*} n$, and $0.40^{*} n$ commodities. We do the same for each Steiner and planar graph but we generate only one instance for each rate of commodity instead of two. Table 4 describes the instances generated, including their numbers of vertices, edges, and the sizes of the commodity sets $T$.

For comparison, we have reimplemented the ACO algorithm described in [18] in the COMET programming language. For each problem instance, the three algorithms ACO, LS-SGA, and LS-R are executed 20 times each. Due to the high complexity of

9 This is different from what we did in [54], where we randomly generated 20 instances for each rate of commodity. For each instance, the algorithm was executed only once. 


\begin{tabular}{|l|r|r|r|}
\hline Name & $|V|$ & $|E|$ & Degree avg. \\
\hline bl-wr2-wht2.10-50.rand & 500 & 1020 & 4.08 \\
bl-wr2-wht2.10-50.sdeg & 500 & 1020 & 4.08 \\
mesh15x15 & 225 & 420 & 3.73 \\
mesh25x25 & 625 & 1200 & 3.84 \\
\hline steinb4.txt & 50 & 100 & 4.00 \\
steinb10.txt & 75 & 150 & 4.00 \\
steinb16.txt & 100 & 200 & 4.00 \\
steinc6.txt & 500 & 1000 & 4.00 \\
steinc11.txt & 500 & 2500 & 10.00 \\
steinc16.txt & 500 & 12500 & 50.00 \\
\hline planar-n50 & 50 & 135 & 5.4 \\
planar-n100 & 100 & 285 & 5.7 \\
planar-n200 & 200 & 583 & 5.83 \\
planar-n500 & 500 & 1477 & 5.91 \\
\hline
\end{tabular}

Table 3 Description of graphs of the benchmarks

the problem, we set the time limit to 30 minutes for each execution. In total, we have 54 problem instances and 1080 executions.

Results The experimental results are shown in Tables 5, 6, and 7. These tables have the same structure, which is described in what follows. The first column presents the instance name. Columns 2-5 present the results of the ACO algorithm [18], including the average, the minimal and the maximal of the best objective values found in 20 executions, and the average time for finding these best objective values. The same information for LS-SGA and LS-R are presented in columns 6-9 and columns 11-14. Column 10 compares the ACO and LS-SGA algorithms in the format $a / b$ where $a$ is the number of times the ACO algorithm found better solutions than the LS-SGA algorithm and $b$ is the number of time the LS-SGA found better solutions than the ACO algorithm in 20 executions. Column 15 presents the same information as column 10 but for the comparison between the ACO and the LS-R algorithms. A comparison of the two algorithms in terms of box-and-whiskers plots (see their template presentation in Figure 15) can be found in Figures 20, 21, 22, 23, 24, and 25. Three consecutive bars present the results computed by the ACO, LS-SGA, and the LS-R algorithms on a given instance. The figures show that for each algorithm, the variance of the results among the 20 executions is small. It also shows that, in most instances, the solutions found by LS-SGA and LS-R are better than those found by the ACO algorithm.

The experiments results show that on average, the LS-R algorithm is better than two other algorithms. The LS-SGA algorithm is better than the ACO algorithm. The LS-SGA finds better solutions than the ACO algorithm in 534 out of 1080 executions while the ACO algorithm finds better solutions in 96 out of 1080 executions. LS$\mathrm{R}$ finds better solutions than ACO in 614 out of 1080 executions while the ACO algorithm finds better solutions than LS-R in 7 out of 1080 executions. 


\begin{tabular}{|c|c|c|c|c|}
\hline index & name & $\sharp V$ & $\sharp E$ & $\sharp T$ \\
\hline 1 & bl-wr2-wht2.10-50.rand.bb_com10_ins1 & 500 & 1020 & 50 \\
\hline 2 & bl-wr2-wht2.10-50.rand.bb_com25_ins1 & 500 & 1020 & 125 \\
\hline 3 & bl-wr2-wht2.10-50.rand.bb_com40_ins1 & 500 & 1020 & 200 \\
\hline 4 & bl-wr2-wht2.10-50.rand.bb_com10_ins2 & 500 & 1020 & 50 \\
\hline 5 & bl-wr2-wht2.10-50.rand.bb_com25_ins2 & 500 & 1020 & 125 \\
\hline 6 & bl-wr2-wht2.10-50.rand.bb_com40_ins2 & 500 & 1020 & 200 \\
\hline 7 & bl-wr2-wht2.10-50.sdeg.bb_com10_ins1 & 500 & 1020 & 50 \\
\hline 8 & bl-wr2-wht2.10-50.sdeg.bb_com25_ins1 & 500 & 1020 & 125 \\
\hline 9 & bl-wr2-wht2.10-50.sdeg.bb_com40_ins1 & 500 & 1020 & 200 \\
\hline 10 & bl-wr2-wht2.10-50.sdeg.bb_com10_ins2 & 500 & 1020 & 50 \\
\hline 11 & bl-wr2-wht2.10-50.sdeg.bb_com25_ins2 & 500 & 1020 & 125 \\
\hline 12 & bl-wr2-wht2.10-50.sdeg.bb_com40_ins2 & 500 & 1020 & 200 \\
\hline 13 & mesh15x15.bb_com10_ins 1 & 225 & 420 & 22 \\
\hline 14 & mesh15x15.bb_com25_ins 1 & 225 & 420 & 56 \\
\hline 15 & mesh15x15.bb_com40_ins 1 & 225 & 420 & 90 \\
\hline 16 & mesh15x15.bb_com10_ins2 & 225 & 420 & 22 \\
\hline 17 & mesh15x15.bb_com25_ins 2 & 225 & 420 & 56 \\
\hline 18 & mesh15x15.bb_com40_ins 2 & 225 & 420 & 90 \\
\hline 19 & mesh25x25.bb_com10_ins 1 & 625 & 1200 & 62 \\
\hline 20 & mesh25x25.bb_com25_ins 1 & 625 & 1200 & 156 \\
\hline 21 & mesh25x25.bb_com40_ins 1 & 625 & 1200 & 250 \\
\hline 22 & mesh25x25.bb_com10_ins2 & 625 & 1200 & 62 \\
\hline 23 & mesh25x25.bb_com 25 ins 2 & 625 & 1200 & 156 \\
\hline 24 & mesh25x25.bb_com40_ins2 & 625 & 1200 & 250 \\
\hline 25 & steinb4.txt_com10_ins1 & 50 & 100 & 5 \\
\hline 26 & steinb4.txt_com25_ins1 & 50 & 100 & 12 \\
\hline 27 & steinb4.txt_com40_ins1 & 50 & 100 & 20 \\
\hline 28 & steinb10.txt_com10_ins 1 & 75 & 150 & 7 \\
\hline 29 & steinb10.txt_com25_ins1 & 75 & 150 & 18 \\
\hline 30 & steinb10.txt_com40_ins1 & 75 & 150 & 30 \\
\hline 31 & steinb16.txt_com10_ins1 & 100 & 200 & 10 \\
\hline 32 & steinb16.txt_com25_ins1 & 100 & 200 & 25 \\
\hline 33 & steinb16.txt_com40_ins1 & 100 & 200 & 40 \\
\hline 34 & steinc6.txt_com10_ins1 & 500 & 1000 & 50 \\
\hline 35 & steinc6.txt_com25_ins1 & 500 & 1000 & 125 \\
\hline 36 & steinc6.txt_com40_ins1 & 500 & 1000 & 200 \\
\hline 37 & steinc11.txt_com10_ins1 & 500 & 2500 & 50 \\
\hline 38 & steinc11.txt_com25_ins1 & 500 & 2500 & 125 \\
\hline 39 & steinc11.txt_com40_ins1 & 500 & 2500 & 200 \\
\hline 40 & steinc16.txt_com10_ins1 & 500 & 12500 & 50 \\
\hline 41 & steinc16.txt_com25_ins1 & 500 & 12500 & 125 \\
\hline 42 & steinc16.txt_com40_ins1 & 500 & 12500 & 200 \\
\hline 43 & planar-n50.ins1.txt_com10_ins1 & 50 & 135 & 5 \\
\hline 44 & planar-n50.ins1.txt_com25_ins1 & 50 & 135 & 12 \\
\hline 45 & planar-n50.ins1.txt_com40_ins1 & 50 & 135 & 20 \\
\hline 46 & planar-n100.ins1.txt_com10_ins1 & 100 & 285 & 10 \\
\hline 47 & planar-n100.ins1.txt_com25_ins1 & 100 & 285 & 25 \\
\hline 48 & planar-n100.ins1.txt_com40_ins1 & 100 & 285 & 40 \\
\hline 49 & planar-n200.ins1.txt_com10_ins1 & 200 & 583 & 20 \\
\hline 50 & planar-n200.ins1.txt_com25_ins1 & 200 & 583 & 50 \\
\hline 51 & planar-n200.ins1.txt_com40_ins1 & 200 & 583 & 80 \\
\hline 52 & planar-n500.ins1.txt_com10_ins1 & 500 & 1477 & 50 \\
\hline 53 & planar-n500.ins1.txt_com25_ins1 & 500 & 1477 & 125 \\
\hline 54 & planar-n500.ins1.txt_com40_ins1 & 500 & 1477 & 200 \\
\hline
\end{tabular}

Table 4 Description of instances 


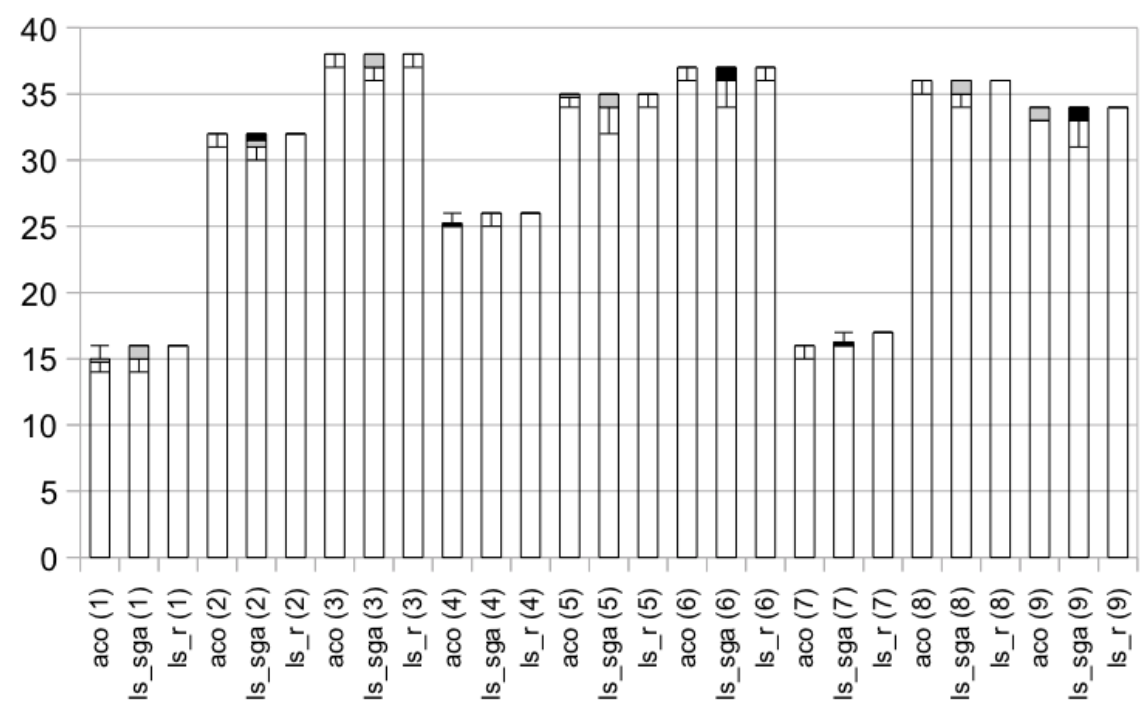

Fig. 20 Comparison between the ACO, LS-SGA and LS-R algorithms (part I)

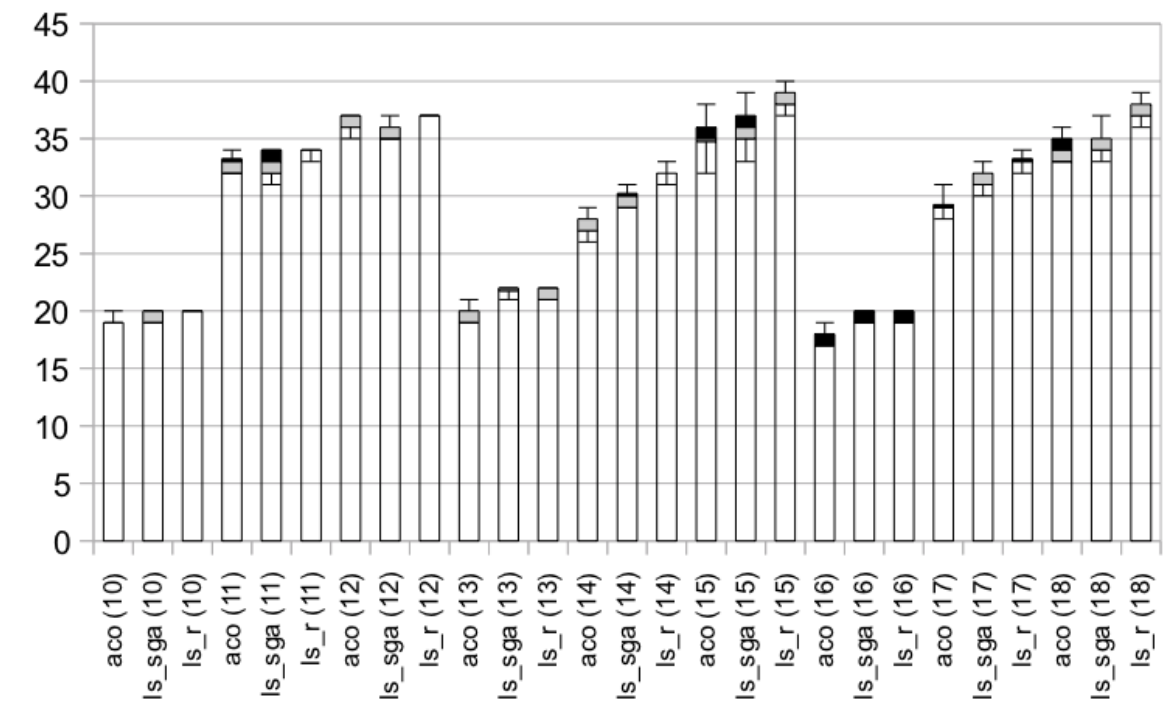

Fig. 21 Comparison between the ACO, LS-SGA and LS-R algorithms (part II) 


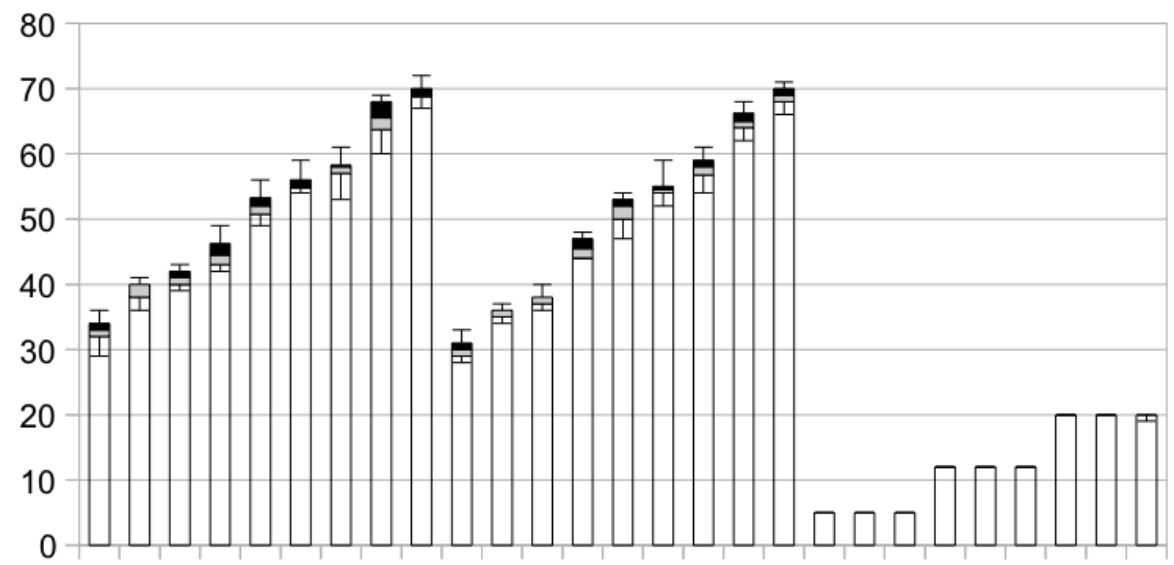

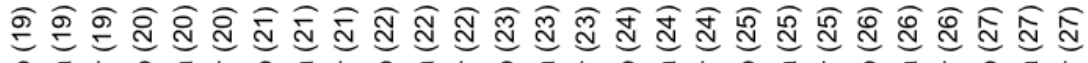

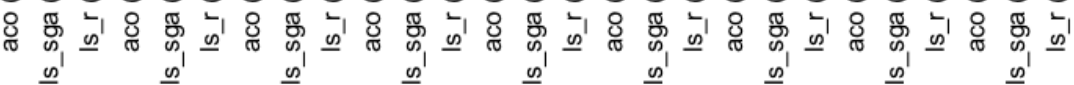

Fig. 22 Comparison between the ACO, LS-SGA and LS-R algorithms (part III)

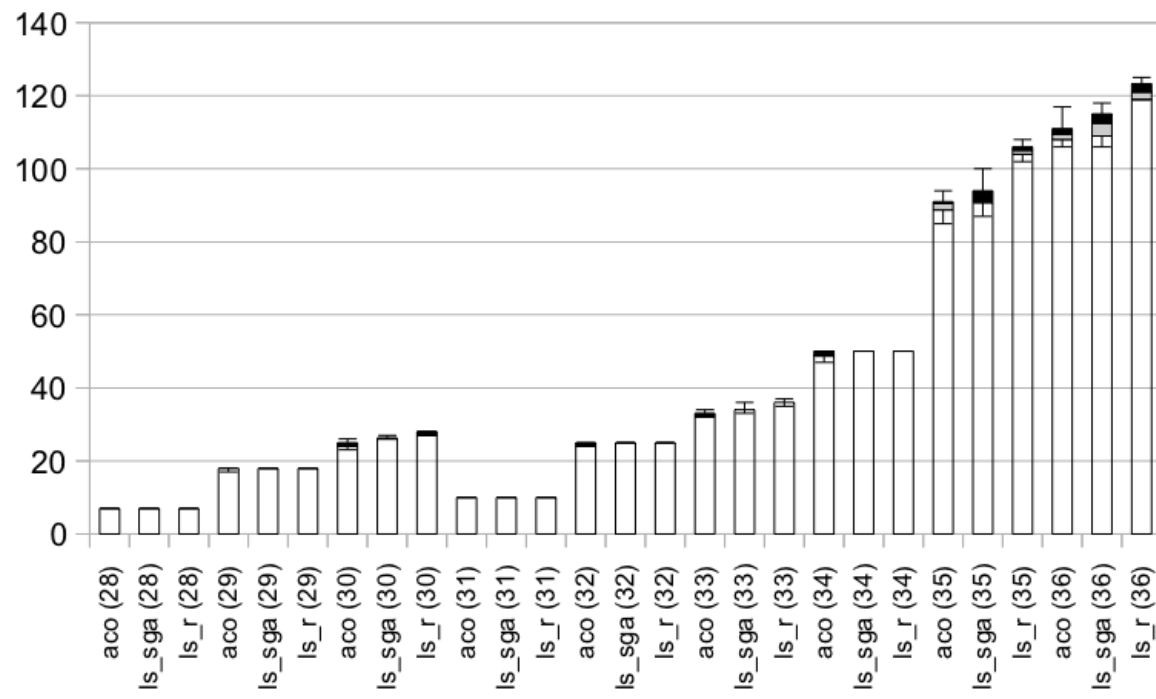

Fig. 23 Comparison between the ACO, LS-SGA and LS-R algorithms (part IV) 


\begin{tabular}{|c|c|c|c|c|c|c|c|c|c|c|c|c|c|c|}
\hline \multirow{2}{*}{ Ins. } & \multicolumn{4}{|c|}{$\mathrm{ACO}$} & \multicolumn{5}{|c|}{ LS-SGA } & \multicolumn{5}{|c|}{ LS-R } \\
\hline & $\bar{f}$ & $m$ & $M$ & $\bar{t}$ & $\bar{f}$ & $m$ & $M$ & $\bar{t}$ & $\tau_{1}$ & $\bar{f}$ & $m$ & $M$ & $\bar{t}$ & $\tau_{2}$ \\
\hline 1 & 14.8 & 14 & 16 & 131.6 & 15.6 & 14 & 16 & 410.76 & $2 / 13$ & 16 & 16 & 16 & 194.71 & $0 / 19$ \\
\hline 2 & 31.85 & 31 & 32 & 165.22 & 31.4 & 30 & 32 & 564.99 & $10 / 3$ & 32 & 32 & 32 & 263.71 & $0 / 3$ \\
\hline 3 & 37.85 & 37 & 38 & 219.56 & 37.6 & 36 & 38 & 322.96 & $6 / 2$ & 37.9 & 37 & 38 & 230.29 & $1 / 2$ \\
\hline 4 & 25.25 & 25 & 26 & 95.41 & 25.9 & 25 & 26 & 434.43 & $0 / 13$ & 26 & 26 & 26 & 151.09 & $0 / 15$ \\
\hline 5 & 34.75 & 34 & 35 & 97.33 & 34.4 & 32 & 35 & 544.02 & $8 / 4$ & 34.95 & 34 & 35 & 303.26 & $0 / 4$ \\
\hline 6 & 36.95 & 36 & 37 & 185.14 & 36.05 & 34 & 37 & 422.18 & $13 / 0$ & 36.95 & 36 & 37 & 293.27 & $1 / 1$ \\
\hline 7 & 15.95 & 15 & 16 & 89.24 & 16.25 & 16 & 17 & 529.03 & $0 / 6$ & 17 & 17 & 17 & 430.99 & $0 / 20$ \\
\hline 8 & 35.8 & 35 & 36 & 67.08 & 35.45 & 34 & 36 & 536.4 & $8 / 3$ & 36 & 36 & 36 & 423.68 & $0 / 4$ \\
\hline 9 & 33.65 & 33 & 34 & 169.9 & 33.1 & 31 & 34 & 472.56 & $11 / 4$ & 34 & 34 & 34 & 557.57 & $0 / 7$ \\
\hline 10 & 19.2 & 19 & 20 & 401.19 & 19.65 & 19 & 20 & 522.22 & $2 / 11$ & 20 & 20 & 20 & 448.59 & $0 / 16$ \\
\hline 11 & 32.95 & 32 & 34 & 365.09 & 32.9 & 31 & 34 & 880.04 & $9 / 7$ & 33.9 & 33 & 34 & 516.21 & $1 / 14$ \\
\hline 12 & 36.5 & 35 & 37 & 133 & 35.7 & 35 & 37 & 936.57 & $11 / 2$ & 37 & 37 & 37 & 583.99 & $0 / 9$ \\
\hline 13 & 19.65 & 19 & 21 & 457.46 & 21.75 & 21 & 22 & 644.11 & $0 / 20$ & 21.55 & 21 & 22 & 360.53 & $0 / 19$ \\
\hline 14 & 27.7 & 26 & 29 & 470.98 & 29.8 & 29 & 31 & 335.51 & $0 / 19$ & 32 & 31 & 33 & 887.93 & $0 / 20$ \\
\hline 15 & 35.3 & 32 & 38 & 871.22 & 35.8 & 33 & 39 & 763.25 & $6 / 10$ & 38.8 & 37 & 40 & 960.97 & $0 / 20$ \\
\hline 16 & 17.5 & 17 & 19 & 479.89 & 19.4 & 19 & 20 & 515.65 & $0 / 19$ & 19.45 & 19 & 20 & 568.74 & $0 / 18$ \\
\hline 17 & 29.2 & 28 & 31 & 1010.52 & 31.7 & 30 & 33 & 480.98 & $0 / 20$ & 33.05 & 32 & 34 & 592.96 & $0 / 20$ \\
\hline 18 & 34 & 33 & 36 & 750.55 & 34.6 & 33 & 37 & 649.26 & $4 / 13$ & 37.6 & 36 & 39 & 910.63 & $0 / 20$ \\
\hline 19 & 32.85 & 29 & 36 & 996.96 & 39.15 & 36 & 41 & 864.6 & $0 / 20$ & 41 & 39 & 43 & 946.47 & $0 / 20$ \\
\hline 20 & 45 & 42 & 49 & 1104.82 & 51.95 & 49 & 56 & 1053.57 & $0 / 20$ & 55.55 & 54 & 59 & 1111.8 & $0 / 20$ \\
\hline 21 & 57.7 & 53 & 61 & 797.14 & 65.3 & 60 & 69 & 950.87 & $0 / 20$ & 69.3 & 67 & 72 & 1520.61 & $0 / 20$ \\
\hline 22 & 30.1 & 28 & 33 & 944.36 & 35.7 & 34 & 37 & 875.12 & $0 / 20$ & 37.9 & 36 & 40 & 945.08 & $0 / 20$ \\
\hline 23 & 45.6 & 44 & 48 & 1015.84 & 51.35 & 47 & 54 & 673.59 & $0 / 20$ & 54.7 & 52 & 59 & 1042.11 & $0 / 20$ \\
\hline 24 & 57.75 & 54 & 61 & 939.82 & 65.05 & 62 & 68 & 1409.13 & $0 / 20$ & 68.85 & 66 & 71 & 1040.24 & $0 / 20$ \\
\hline
\end{tabular}

Table 5 Experimental results of the first graphs set

\begin{tabular}{|c|c|c|c|c|c|c|c|c|c|c|c|c|c|c|}
\hline \multirow{2}{*}{ Ins. } & \multicolumn{4}{|c|}{$\mathrm{ACO}$} & \multicolumn{5}{|c|}{ LS-SGA } & \multicolumn{5}{|c|}{ LS-R } \\
\hline & $\bar{f}$ & $m$ & $M$ & $\bar{t}$ & $\bar{f}$ & $m$ & $M$ & $\bar{t}$ & $\tau_{1}$ & $\bar{f}$ & $m$ & $M$ & $\bar{t}$ & $\tau_{2}$ \\
\hline 25 & 5 & 5 & 5 & 0.01 & 5 & 5 & 5 & 1.12 & $0 / 0$ & 5 & 5 & 5 & 1.09 & $0 / 0$ \\
\hline 26 & 12 & 12 & 12 & 0.44 & 12 & 12 & 12 & 1.22 & $0 / 0$ & 12 & 12 & 12 & 1.4 & $0 / 0$ \\
\hline 27 & 20 & 20 & 20 & 51.11 & 20 & 20 & 20 & 5.45 & $0 / 0$ & 19.9 & 19 & 20 & 2.8 & $2 / 0$ \\
\hline 28 & 7 & 7 & 7 & 0.02 & 7 & 7 & 7 & 1.35 & $0 / 0$ & 7 & 7 & 7 & 1.16 & $0 / 0$ \\
\hline 29 & 17.85 & 17 & 18 & 96.48 & 18 & 18 & 18 & 13.42 & $0 / 3$ & 18 & 18 & 18 & 5.2 & $0 / 3$ \\
\hline 30 & 24.35 & 23 & 26 & 242.04 & 26.25 & 26 & 27 & 682.84 & $0 / 20$ & 27.3 & 27 & 28 & 505.22 & $0 / 20$ \\
\hline 31 & 10 & 10 & 10 & 0.25 & 10 & 10 & 10 & 1.46 & $0 / 0$ & 10 & 10 & 10 & 1.52 & $0 / 0$ \\
\hline 32 & 24.35 & 24 & 25 & 364.99 & 25 & 25 & 25 & 93.53 & $0 / 13$ & 25 & 25 & 25 & 8.86 & $0 / 13$ \\
\hline 33 & 32.45 & 32 & 34 & 658.25 & 34.1 & 33 & 36 & 747.34 & $0 / 19$ & 35.95 & 35 & 37 & 646.19 & $0 / 20$ \\
\hline 34 & 49.1 & 47 & 50 & 572.75 & 50 & 50 & 50 & 184.44 & $0 / 12$ & 50 & 50 & 50 & 240.75 & $0 / 12$ \\
\hline 35 & 89.9 & 85 & 94 & 728.76 & 92.2 & 87 & 100 & 734.88 & $4 / 12$ & 104.95 & 102 & 108 & 1370.88 & $0 / 20$ \\
\hline 36 & 109.8 & 106 & 117 & 924.1 & 112.05 & 106 & 118 & 971.4 & $2 / 14$ & 121.4 & 119 & 125 & 1372.37 & $0 / 20$ \\
\hline 37 & 50 & 50 & 50 & 23.59 & 50 & 50 & 50 & 42.19 & $0 / 0$ & 50 & 50 & 50 & 37.64 & $0 / 0$ \\
\hline 38 & 123.3 & 122 & 125 & 521.8 & 125 & 125 & 125 & 128.49 & $0 / 17$ & 125 & 125 & 125 & 262.38 & $0 / 17$ \\
\hline 39 & 194.25 & 190 & 198 & 494.64 & 200 & 200 & 200 & 395.54 & $0 / 20$ & 200 & 200 & 200 & 473.81 & $0 / 20$ \\
\hline 40 & 50 & 50 & 50 & 6.89 & 50 & 50 & 50 & 55.12 & $0 / 0$ & 50 & 50 & 50 & 46.01 & $0 / 0$ \\
\hline 41 & 125 & 125 & 125 & 17.13 & 125 & 125 & 125 & 194.36 & $0 / 0$ & 125 & 125 & 125 & 113.83 & $0 / 0$ \\
\hline 42 & 200 & 200 & 200 & 45.32 & 200 & 200 & 200 & 366.69 & $0 / 0$ & 200 & 200 & 200 & 183.32 & $0 / 0$ \\
\hline
\end{tabular}

Table 6 Experimental results of the steiner graphs set

6.3 The routing and wavelength assignment problem with delay side constraint (RWA-D)

The last application demonstrates that VarPath variables of LS (Graph) and var $\{$ int $\}$ of COMET can easily be combined. 


\begin{tabular}{|c|c|c|c|c|c|c|c|c|c|c|c|c|c|c|}
\hline \multirow{2}{*}{ Ins. } & \multicolumn{4}{|c|}{$\mathrm{ACO}$} & \multicolumn{5}{|c|}{ LS-SGA } & \multicolumn{5}{|c|}{ LS-R } \\
\hline & $\bar{f}$ & $m$ & $M$ & $\bar{t}$ & $\bar{f}$ & $m$ & $M$ & $\bar{t}$ & $\tau_{1}$ & $\bar{f}$ & $m$ & $M$ & $\bar{t}$ & $\tau_{2}$ \\
\hline 43 & 5 & 5 & 5 & 0.03 & 5 & 5 & 5 & 0.86 & $0 / 0$ & 5 & 5 & 5 & 0.8 & $0 / 0$ \\
\hline 44 & 12 & 12 & 12 & 0.16 & 12 & 12 & 12 & 0.96 & $0 / 0$ & 12 & 12 & 12 & 0.97 & $0 / 0$ \\
\hline 45 & 20 & 20 & 20 & 36.38 & 20 & 20 & 20 & 25.12 & $0 / 0$ & 19.9 & 19 & 20 & 31.18 & $2 / 0$ \\
\hline 46 & 10 & 10 & 10 & 0.12 & 10 & 10 & 10 & 1.14 & $0 / 0$ & 10 & 10 & 10 & 1.07 & $0 / 0$ \\
\hline 47 & 25 & 25 & 25 & 20.22 & 25 & 25 & 25 & 7.05 & $0 / 0$ & 25 & 25 & 25 & 5.33 & $0 / 0$ \\
\hline 48 & 34 & 33 & 36 & 680.72 & 35.3 & 34 & 37 & 813.56 & $0 / 16$ & 36 & 35 & 37 & 698.88 & $0 / 18$ \\
\hline 49 & 20 & 20 & 20 & 13.46 & 20 & 20 & 20 & 4.06 & $0 / 0$ & 20 & 20 & 20 & 5.23 & $0 / 0$ \\
\hline 50 & 41.8 & 39 & 43 & 889.07 & 44.85 & 43 & 47 & 988.81 & $0 / 20$ & 45.95 & 45 & 48 & 853.18 & $0 / 20$ \\
\hline 51 & 49.35 & 47 & 51 & 790.65 & 53.35 & 51 & 56 & 1033.97 & $0 / 19$ & 55.7 & 54 & 58 & 901.74 & $0 / 20$ \\
\hline 52 & 44.95 & 42 & 47 & 1100.41 & 49.95 & 49 & 50 & 484.84 & $0 / 20$ & 50 & 50 & 50 & 309.24 & $0 / 20$ \\
\hline 53 & 60.95 & 57 & 65 & 954.35 & 73.85 & 70 & 77 & 1345.74 & $0 / 20$ & 78.2 & 77 & 80 & 1044.03 & $0 / 20$ \\
\hline 54 & 82.85 & 78 & 86 & 1235.13 & 93.95 & 91 & 99 & 1366.27 & $0 / 20$ & 100.15 & 97 & 102 & 1455.43 & $0 / 20$ \\
\hline
\end{tabular}

Table 7 Experimental results of the planar graphs set

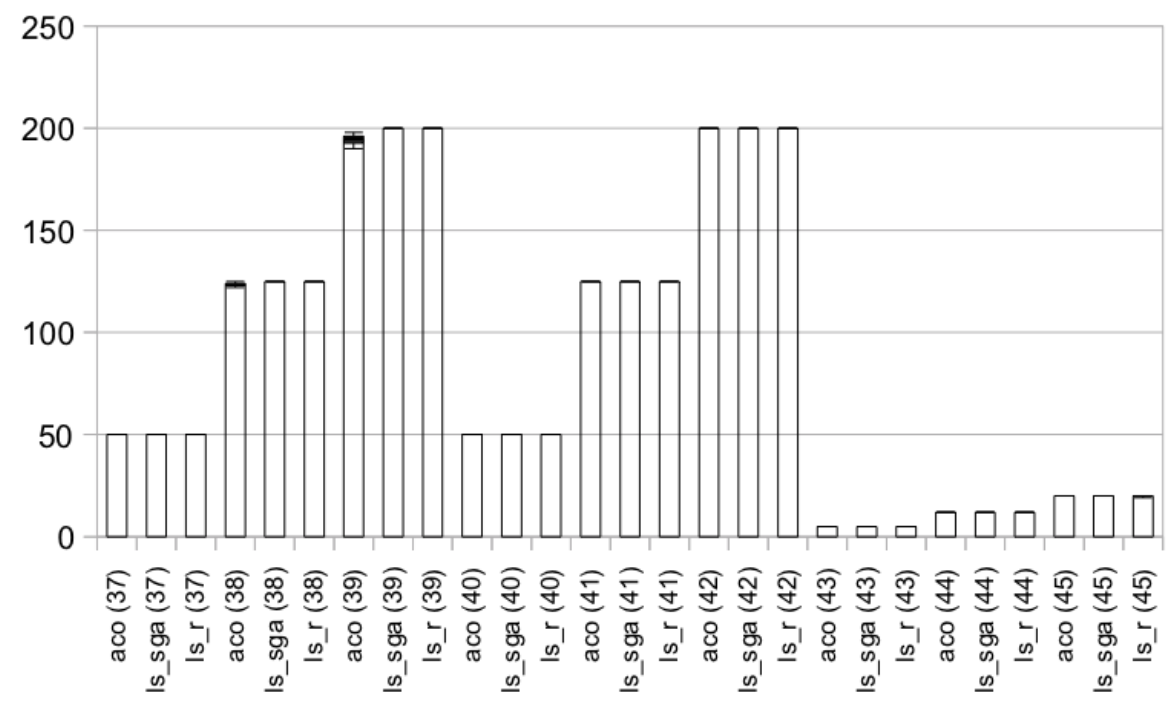

Fig. 24 Comparison between the ACO, LS-SGA and LS-R algorithms (part V)

\subsubsection{Problem statement}

Given an undirected weighted graph $G=(V, E)$, each edge $e$ of $G$ has cost $c(e)$ (e.g., the delay in traversing e). Suppose given a set of connection requests $R=$ $\left\{\left\langle s_{1}, t_{1}\right\rangle,\left\langle s_{2}, t_{2}\right\rangle, \ldots,\left\langle s_{k}, t_{k}\right\rangle\right\}$ and a value $D$. The RWA-D problem consists of finding routes $p_{i}$ from $s_{i}$ to $t_{i}$ and their wavelengths for all $i=1,2, \ldots, k$ such that:

1. the wavelengths of $p_{i}$ and $p_{j}$ are different if they have common edges, $\forall i \neq j \in$ $\{1,2, \ldots, k\}$ (wavelength constraint),

2. $\sum_{e \in p_{i}} c(e) \leq D, \forall i=1,2, \ldots, k$ (delay constraint),

3 . the number of different wavelengths is minimized (objective function). 


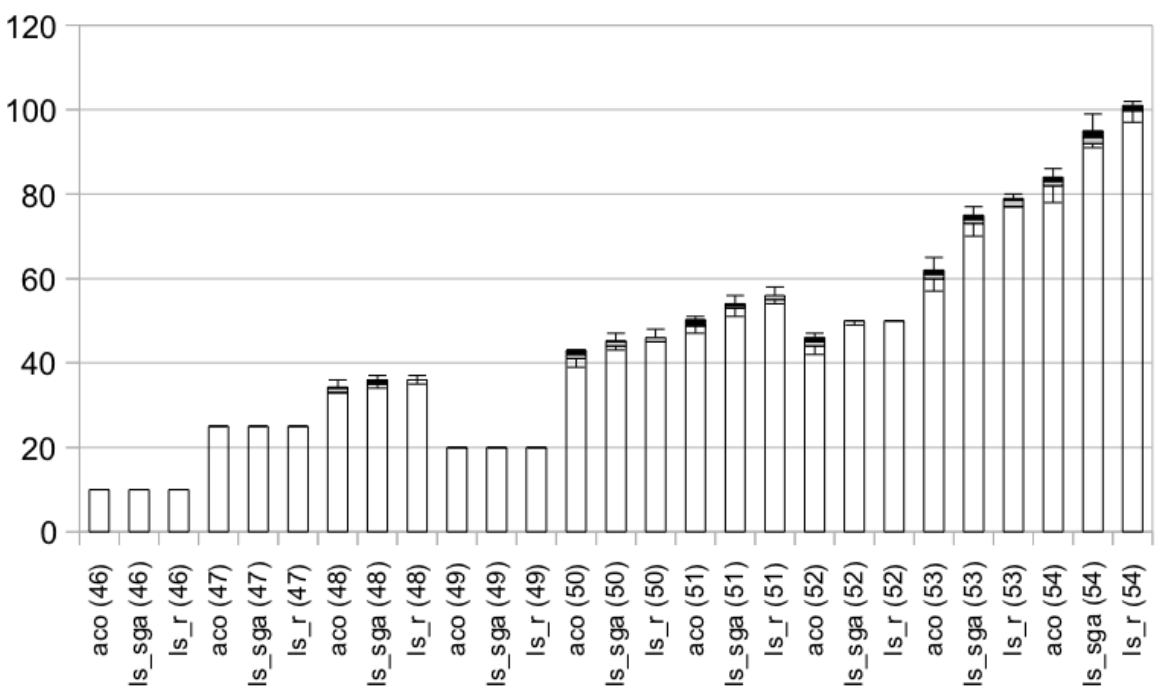

Fig. 25 Comparison between the ACO, LS-SGA and LS-R algorithms (part VI)

\subsubsection{The model}

The idea of the proposed algorithm is simple. We iteratively perform a local search algorithm for finding a feasible solution to the RWA-D problem with $W$ wavelengths $(W=1,2,3, \ldots)$ until the first feasible solution is discovered.

The model is given in Figure 26. Lines 4-10 initialize all VarPath vps [i] from $s$ [i] to $t$ [i] with the shortest version. Line 11 initializes an array vw where Vw [i] stores the wavelength value for the path vps [i]. The search starts with one wavelength (see line 14). At each step, we try to find a feasible solution to the RWAD problem by a localsearch procedure (line 16). The search terminates (line 17) if a feasible solution to the RWA-D problem is discovered, otherwise, we increase $W$ by one (line 19).

The local search procedure described in Figure 27 receives an array of VarPath vps, a value $W$ of the number of wavelengths, and local search parameters maxIt and $\operatorname{maxT}$ as input. Line 2 creates a Solver<LSGraph> 1 s and lines 4 6 post all VarPath to it. Line 8 initializes an array var $\{i n t\} x w$, where $x w[i]$ represents the wavelength assigned to the path vps [i] and is initialized with the value $\mathrm{Vw}[i]$. The domain of $\mathrm{xw}$ [ $i$ ] is 1 . .W. Line 10 initializes a Constraint $S-$ ystem<LSGraph> CS. The first constraint of the RWA-D problem is stated and posted in line 12. Lines 14-15 state and post all side constraints (the delay constraint) to CS and line 17 closes the constraint system CS. Line 19 groups all variables vps, $\mathrm{xw}$, and the constraint CS, into a model mod. Line 20 creates a search component which will be given in detail in Figure 28. Lines 22-23 set parameters for the search and line 25 performs the search. The value of $\mathrm{xw}$ is stored in $\mathrm{Vw}$ for the next iteration (see lines 27-28): all paths vps [i ] and their wavelengths xw [i] are conserved for 


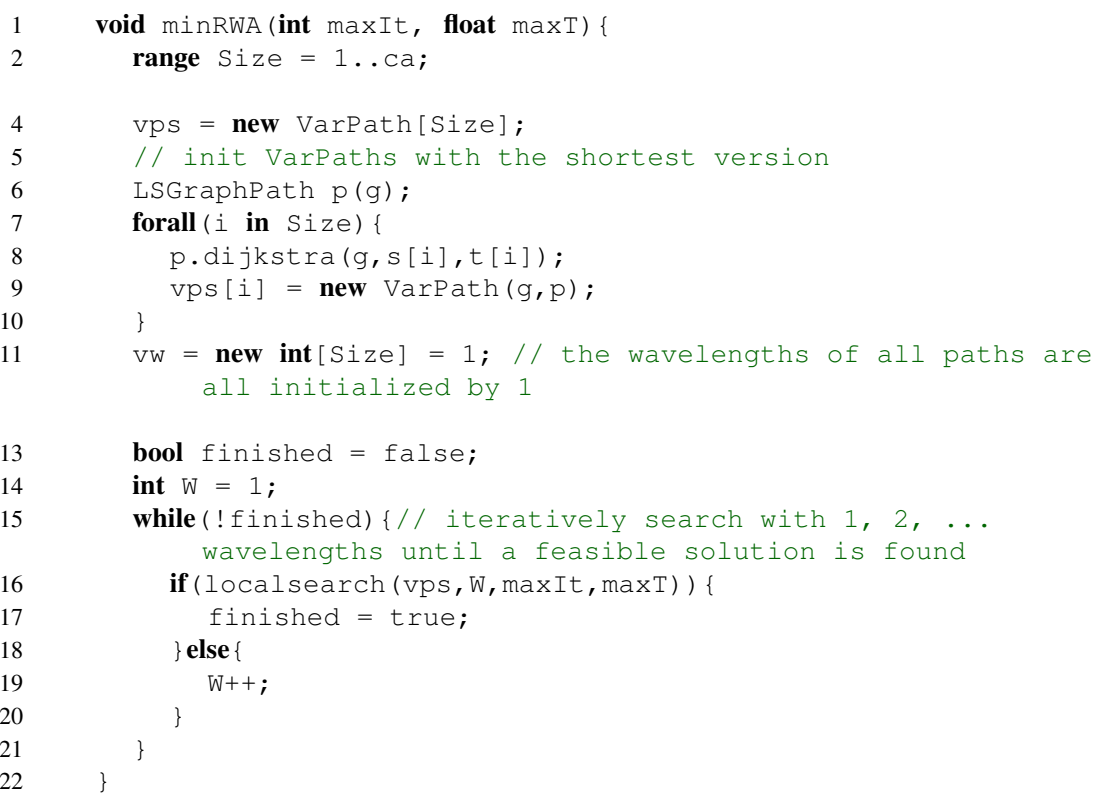

Fig. 26 Model for the RWA-D problem

the next localsearch. The localsearch returns true if a feasible solution to the RWA-D problem is discovered (lines 30-32).

The search component is given in Figure 28. It extends the TabuSearch $<\mathrm{LSG}-$ raph $>$ and receives Lmax (line 3) as parameters for the solution initialization when restarting the tabu search. The restartSolution is overriden (lines 13-24) in which we initialize the value for the VarPath vps [i] with the shortest version if its cost is greater than Lmax. This aims at quickly satisfying the delay constraint. The initsolution is also overriden, which does nothing in order not to change the value of the variables computed in the previous step of the search. The search explores two neighborhoods (lines 7-10) (see [53] for details about these neighborhood explorations).

\subsubsection{Naive greedy algorithm}

As far as we know, the RWA-D problem has not been considered before. In order to assess the efficiency of our local search, we implement a simple greedy heuristic algorithm for the RWA-D problem (see Algorithm 8). The main idea of this greedy heuristic is to find the shortest path ${ }^{10}$ for each connection request and assigns a wavelength to this connection request in a greedy way without violating the wavelength constraint. Variable $S o l$ in line 1 represents the set of paths under construction. Variable $W$ (line 2) contains the set of wavelengths used for the paths which have already

10 The shortest path best ensures satisfaction of the delay constraint. 
1

4

5

10

12

14

15

17

19

20

22

23

25

27

28

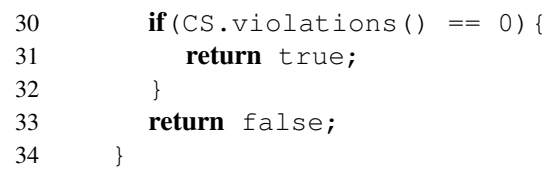

forall ( $i$ in $\operatorname{vps} . \operatorname{rng}())$ \{ ls.post (vps [i]); $\mathrm{xw}[j]$.

forall ( $i$ in vps.rng( $)$ ) delay constraint

CS.close ();

se. setMaxIter (maxIt);

se. setMaxTime $(\operatorname{maxT})$;

forall ( $i$ in $x w \cdot r n g())$ wavelengths bool localsearch (Varpath[] vps, int $W$, int maxIt, float maxT) $\{/ /$ try to find a feasible solution with $W$ wavelengths Solver<LSGraph> Is(); // create a new solver

$\mathrm{xW}=\operatorname{new} \operatorname{var}\{$ int $\}[i$ in $\operatorname{vps} . r n g()](1 \mathrm{~s}, 1 \ldots \mathrm{W}):=\mathrm{vw}[\mathrm{i}] ; / /$ initial wavelengths of paths (decision variables) using the values computed at the previous iteration. At this point, the domains of wavelengths are extended from 1..W-1 (at the previous iteration) to $1 \ldots$ W

ConstraintSystem<LSGraph> CS(Is); // constraint system

CS.post (AllDistinctLightPaths (vps, xw)); // posting the constraint specifying that two paths vps[i] and vps[j] sharing a link must have different wavelengths xw[i] and

CS.post (PathCostonEdges (vps[i]) <= Lmax); // posting the

Model<LSGraph> mod(vps, xw, CS) ; // encapsulate variables, constraints into a model object

RWASearch se(mod, Lmax); // create the search object which extends the generic built-in tabu search component

se.search(); // perform the local search

$\mathrm{Vw}[i]=\mathrm{xw}[\mathrm{i}] ; / /$ store the wavelengths of paths computed for the next search iteration with higher number of

Fig. 27 The local search procedure for the RWA-D problem 


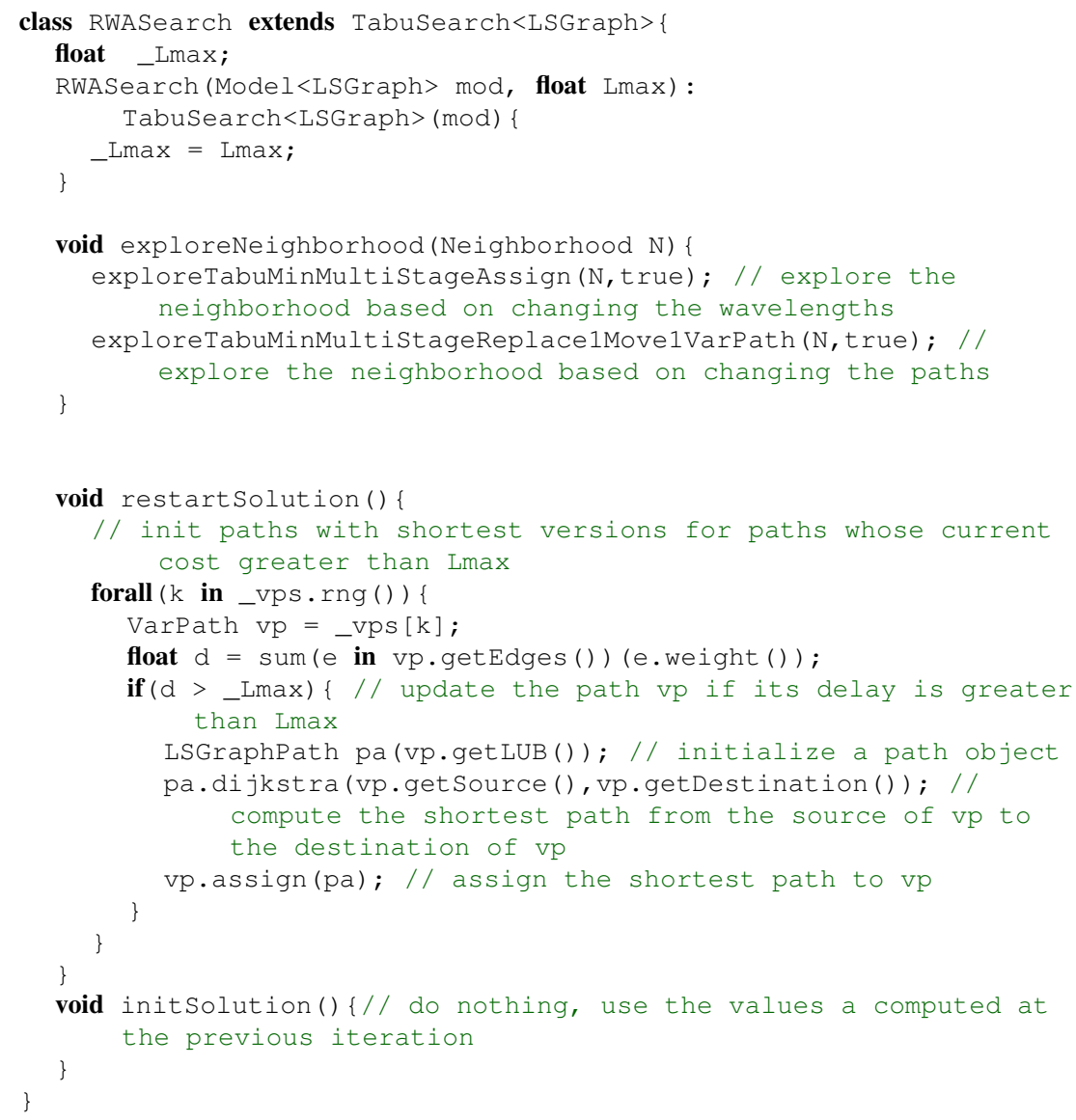

Fig. 28 The search component

been constructed. Variable $n b W$ avelengths (line 3) is the number of wavelengths used. For each connection request $\left\langle s_{i}, t_{i}\right\rangle$ (line 4), we assign the shortest path $P_{i}$ to it (line 6). Variable $W_{i}$ in line 5 represents the candidate wavelengths for $P_{i}$. Lines 7-9 remove all impossible wavelengths for $P_{i}$ from $W_{i}$. If no wavelength already used is possible for $P_{i}$ (line 10), then we have to find a new wavelength $w_{i}$ for $P_{i}$ (lines 11-12). If the candidate set $W_{i}$ is not null, we select randomly a wavelength from $W_{i}$ and assign it to $P_{i}$ (line 15). Lines 16-17 update the solution.

\subsubsection{Experiments}

We compare our local search model with the naive greedy algorithm described in Algorithm 8 (multistart version with 1000 different orders of $\left\langle s_{i}, t_{i}\right\rangle$ to be considered).

The two algorithms have been tried on different instances (graphs from 16 nodes and 33 edges to 100 nodes and 261 edges and with 10,20, and 50 connection requests 


\begin{tabular}{|l|r|r|r|r|r|}
\hline Instances & $g r e e d y$ & f_min & $f \_$max & \multicolumn{1}{c|}{} & \multicolumn{1}{c|}{} \\
\hline arpanet_ca10.ins1 & 5 & 2 & 2 & 2 & 2.15 \\
arpanet_ca20.ins1 & 9 & 6 & 7 & 6.05 & 11.36 \\
arpanet_ca50.ins1 & 16 & 8 & 9 & 8.3 & 68.35 \\
grid_ext_4x4_ca10.ins1 & 3 & 2 & 2 & 2 & 1.62 \\
grid_ext_4x4_ca20.ins1 & 9 & 4 & 5 & 4.3 & 7.16 \\
grid_ext_4x4_ca50.ins1 & 24 & 8 & 10 & 8.55 & 55.04 \\
grid_ext_5x5_ca10.ins1 & 4 & 2 & 2 & 2 & 2.43 \\
grid_ext_5x5_ca20.ins1 & 7 & 2 & 3 & 2.95 & 6.21 \\
grid_ext_5x5_ca50.ins1 & 21 & 5 & 8 & 6.45 & 54.15 \\
grid_ext_6x6_ca10.ins1 & 3 & 2 & 2 & 2 & 2.58 \\
grid_ext_6x6_ca20.ins1 & 4 & 2 & 3 & 2.1 & 6.66 \\
grid_ext_6x6_ca50.ins1 & 20 & 5 & 6 & 5.35 & 58.99 \\
grid_ext_8x8_ca10.ins1 & 4 & 2 & 2 & 2 & 3.81 \\
grid_ext_8x8_ca20.ins1 & 9 & 3 & 4 & 3.3 & 14.61 \\
grid_ext_8x8_ca50.ins1 & 11 & 4 & 6 & 5.15 & 73.97 \\
grid_ext_10x10_ca10.ins1 & 4 & 2 & 4 & 2.7 & 9.71 \\
grid_ext_10x10_ca20.ins1 & 8 & 3 & 5 & 4 & 24.22 \\
grid_ext_10x10_ca50.ins1 & 13 & 4 & 6 & 4.75 & 105.2 \\
\hline
\end{tabular}

Table 8 Experimental results for the RWA-D problem

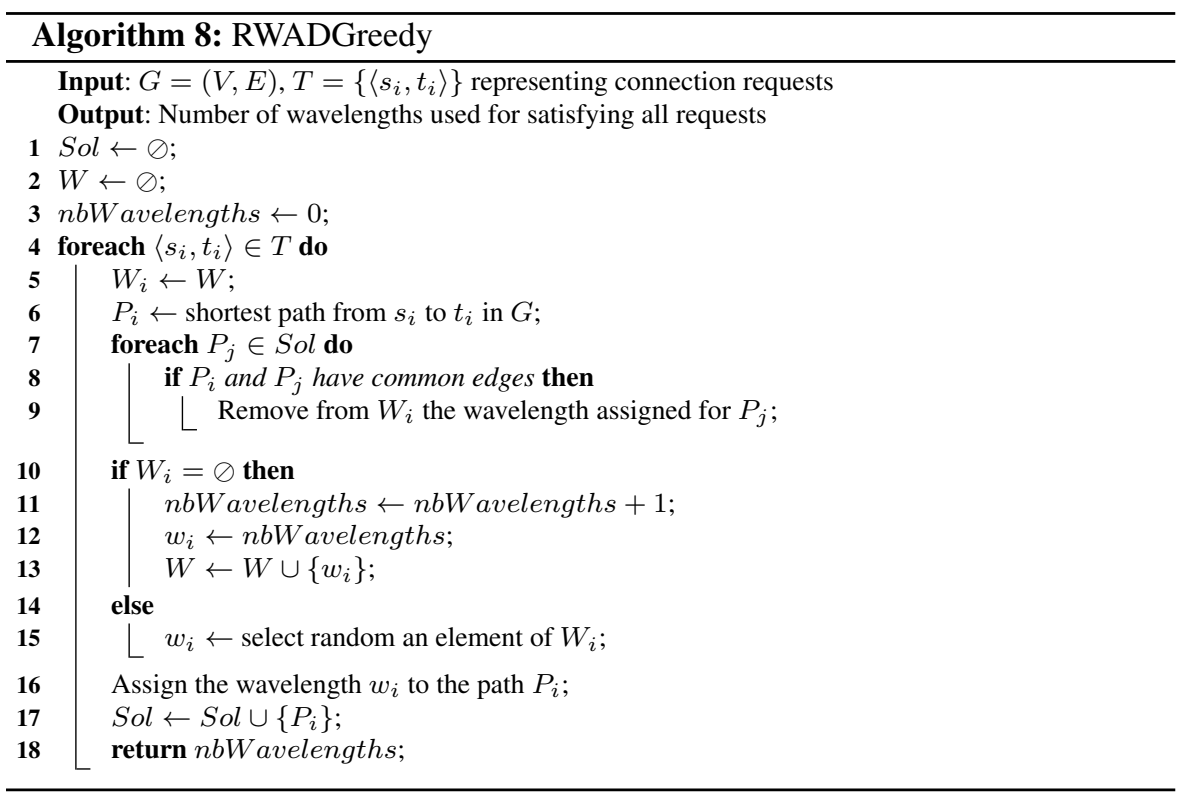

for each graph). Due to the complexity of the problem, we set the number of iterations for the tabu search (the value of maxIt in line 16 of Figure 26) to 200. For each problem instance, the model is executed 20 times. From our preliminary results, we set the length of the tabu lists tbl to 5 and the value of maxStable to 20 .

Table 8 shows the experimental results. Column 2 presents the objective values found by the naive greedy algorithm. Columns 3-6 show the minimal, the maximal, and the average of the best objective value found, and the average execution time (in seconds) over 20 runs. The experimental results show that the local search gives better solutions than the naive greedy algorithm. Especially when the number of connection 
requests increases (i.e., with 50 connection requests), the results found by the local search are two or three times better than those found by the naive greedy algorithm. We can see that the number of wavelengths used increases when the number of connection requests increases. Given a number of connection requests, if the size of the graph increases, then the number of wavelengths used decreases due to the fact that on larger graphs, each link is shared by fewer paths of the solution found by the local search and if two paths are completely edge-disjoint, they can be assigned the same wavelength. For instance, with 50 connection requests, on the graph of 100 vertices, we needed to use only four wavelengths (line 18), while on the graph of 16 vertices, we had to use eight wavelengths (line 6).

Once again, in the above model, we notice that it is easy to state and post various built-in COMET constraints over var $\{$ int $\}$ to the graph constraint system CS, which shows the flexibility and compositionality of the framework.

\section{Conclusion}

This paper considered constrained optimum trees and paths (COT/COP) problems which arise in many real-life applications. It proposed a domain-specific constraintbased local search (CBLS) framework (called LS (Graph) ) for solving COT/COP applications, enabling models to be high level, compositional, and extensible, and allowing for a clear separation between model and search. The key technical contribution to support the COP framework is a novel neighborhood based on a rooted spanning tree that implicitly defines a path between the source and the target and its neighbors, and provides an efficient data structure for differentiation. The paper proved that the neighborhood obtained by swapping edges in this tree is connected and presented a larger neighborhood involving multiple independent moves. The LS (Graph) framework, implemented in COMET, was applied to the quorumcast routing problem, the edge-disjoint paths problem, and the routing and wavelength assignment problem with side constraints on optical networks. Computational results showed the potential significance of the approach, both from a modeling and a computational standpoint.

Our future work will focus on the construction of a generic constraint programming (CP) framework and a hybrid system combining CP and CBLS for modeling and solving COT/COP problems.

Acknowledgements We would like to thank the reviewers for their helpful comments and suggestions. This research is partially supported by the Interuniversity Attraction Poles Programme (Belgian State, Belgian Science Policy) and the FRFC project 2.4504.10 of the Belgian FNRS (National Fund for Scientific Research).

\section{References}

1. U. Acar, G. Blelloch, R. Harper, J. Vittes, and S. Woo. An experimental analysis of change propagation in dynamic trees. In proc. 15th SODA, pages 524-533, 2004.

2. R. K. Ahuja, T. L. Magnanti, and J. B. Orlin. Network Flows: Theory, Algorithms, and Applications. Prentice Hall, New Jersey, United States, 1993. 
3. R. K. Ahuja, J. B. Orlinb, and D. Sharma. A composite very large-scale neighborhood structure for the capacitated minimum spanning tree problem. Operations Research Letters 31, pages 185-194, 2003.

4. M. Ali, B. Ramamurthy, and J. Deogun. Genetic algorithm for routing in wdm optical networks with power considerations. part i: The unicast case. In Proceedings of the 8th IEEE ICCCNÕ99, BostonNatick, MA, USA, pages 335-340, 1999.

5. M. Ali, B. Ramamurthy, and J. Deogun. Routing and wavelength assignment with power considerations in optical networks. Computer Networks, 32:539-555, 2000.

6. S. Alstrup, J. Holm, K. D. Lichtenberg, and M. Thorup. Maintaining information in fully dynamic trees with top trees. ACM Trans. Algorithms, 1(2):243-264, 2005.

7. R. Andrade, A. Lucena, and N. Maculan. Using lagrangian dual information to generate degree constrained spanning trees. Discrete Applied Mathematics, pages 703-717, 2006.

8. B. Awerbuch, R. Gawlick, T. Leighton, and Y. Rabani. On-line admission control and circuit routing for high performance computing and communication. In 35th IEEE Symposium on Foundations of Computer Science (FOCS1994), pages 412-423, 1994.

9. P. Badeau, M. Gendreau, F. Guertin, J.-Y. Potvin, and E. Taillard. A parallel tabu search heuristic for the vehicle routing problem with time windows. Transportation Research - C 5, pages 109-122, 1997.

10. D. Banerjee, V. Mehta, and S. Pandey. A genetic algorithm approach for solving the routing and wavelength assignment problem in wdm networks. 3rd IEEE/IEE International Conference on Networking, ICNÕ2004, Paris, pages 70-78, 2004.

11. D. Banerjee and B. Mukherjee. Wavelength-routed optical networks: Linear formulation, resource budgeting tradeoffs, and a reconfiguration study. IEEE/ACM Transactions on Networking, 8:598607,2000 .

12. S. Banerjee, J. Yoo, and C. Chen. Design of wavelength routed optical networks for packet switched traffic. IEEE Journal of Lightware Technology, 15(9):1636-1646, 1997.

13. A. Baveja and A. Srinivasan. Approximation algorithms for disjoint paths and related routing and packing problems. Math. Oper. Res., 25(2):255-280, 2000.

14. J. E. Beasley. Or-library, url=http://people.brunel.ac.uk/ mastjjb/jeb/info.html

15. J. E. Beasley and N. Christofides. An algorithm for the resource constrained shortest path problem. Network, vol. 19, pages 379-394, 1989.

16. M. A. Bender, M. Farach-Colton, G. Pemmasani, S. Skiena, and P. Sumazin. Lowest common ancestors in trees and directed acyclic graphs. Journal of Algorithms 57, pages 75-94, 2005.

17. O. Berkman and U. Vishkin. Recursive star-tree parallel data structure. SIAM J. Comput., 22(2):221242, 1993 .

18. M. Blesa and C. Blum. Finding edge-disjoint paths in networks: An ant colony optimization algorithm. Journal of Mathematical Modelling and Algorithms, 6(3), pages 361-391, 2007.

19. C. Blum. A new hybrid evolutionary algorithm for the huge k-cardinality tree problem. Proceedings of the 8th annual conference on Genetic and evolutionary computation, pages 515-522, 2006.

20. C. Blum and M. Blesa. New metaheuristic approaches for the edge-weighted k-cardinality tree problem. Computers and Operations Research, pages 32(6):1355-1377, 2005

21. T. Bui and G. Sundarraj. Ant system for the k-cardinality tree problem. In K. Deb et al., editor, Proceedings of the Genetic and Evolutionary Computation Conference (GECCO 2004), 3102:36-47, 2004.

22. C. Chekuri and S. Khanna. Edge disjoint paths revisited. In Proceedings of the 14th ACM-SIAM Symposium on Discrete Algorithms (SODA2003), pages 628-637, 2003.

23. C. Chen and S. Banerjee. A new model for optimal routing and wavelength assignment in wavelength division multiplexed optical networks. INFOCOM 1996, pages 164-171, 1996.

24. S. Y. Cheung and A. Kumar. Efficient quorumcast routing algorithms. In: Proceedings of INFOCOM'94, pages 840-847, 1994.

25. M. Chimani, M. Kandyba, and P. M. I. Ljubic. Obtaining optimal k-cardinality trees fast. $A C M$ Journal of Experimental Algorithmics, 14(2):5.1-5.23, 2009.

26. I. Chlamtac, A. Ganz, and G. Karmi. Lightpath communications: An approach to high bandwidth optical WANS. IEEE Transactions on Communications, 40(7):1171-1182, 1992.

27. J. C. N. Clímaco, J. M. F. Craveirinha, and M. M. B. Pascoal. A bicriterion approach for routing problems in multimedia networks. Networks, 41:206-220, 2003.

28. M. de Aragão, E. Uchoa, and R. Werneck. Dual heuristics on the exact solution of large Steiner problems. In Proceedings of the Brazilian Symposium on Graphs, Algorithms and Combinatorics GRACO'01, Fortaleza, 2001. 
29. B. Du, J. Gu, D. Tsang, and W. Wang. Quorumcast routing by multispace search. Proceedings of IEEE Globecom1996, pages 1069-1073, 1996.

30. I. Dumitrescu and N. Boland. Improved preprocessing, labeling and scaling algorithms for the weightconstrained shortest path problem. Networks, 42:135-153, 2003.

31. R. Dutta and G. N. Rouskas. A survey of virtual topology design algorithms for wavelength routed optical networks. Optical Networks, 1(1):73-89, 2000.

32. T. Fischer. Improved local search for large optimum communication spanning tree problems. In MIC'2007 - 7th Metaheuristics International Conference, 2007.

33. G. N. Frederickson. A data structure for dynamically maintaining rooted trees. Journal of Algorithms, 24(1):37-65, 1997.

34. B. Funke, T. Grünert, and S. Irnich. Local search for vehicle routing and scheduling problems: Review and conceptual integration. Journal of Heuristics, 11(4):267-306, 2005.

35. M. Gruber, J. van Hemert, and G. Raidl. Neighborhood searches for the bounded diameter minimum spanning tree problem embedded in a vns, ea, and aco. Proceedings of the Genetic and Evolutionary Computation Conference, pages 1187-1194, 2006.

36. M. R. Henzinger and V. King. Randomized fully dynamic graph algorithms with polylogarithmic time per operation. J ACM, 46(4):502-516, 1999.

37. V. Ho, P. Francois, Y. Deville, D. Pham, and O. Bonaventure. Using local search for traffic engineering in switched ethernet networks. In Proceedings of 22nd International Teletraffic Congress (ITC-22) September 2010, Amsterdam, Netherlands, 2010.

38. E. Hyytia. Resource allocation and performance analysis problems in optical networks, ph.d. thesis In Dpt. of Electrical and Communications Engineering, Helsinki University of Technology, Helsinki, Sweden, 2004

39. B. Jaumard, C. Meyer, and B. Thiongane. Comparison of ilp formulations for the rwa problem. Optical Switching and Networking, 4:157-172, 2007.

40. B. Jaumard, C. Meyer, and X. Yu. How much wavelength conversion allows a reduction in the blocking rate? Journal of Optical Networking, 5(12):881-900, 2006.

41. P.-C. Kanellakis and C. H. Papadimitriou. Local search for the asymmetric traveling salesman problem. OPERATIONS RESEARCH, 28(5):1086-1099, 1980.

42. J. Kleinberg. Approximation algorithms for disjoint-paths problems. PhD thesis. The MIT Press, Cambridge, USA, 1996.

43. S. G. Kolliopoulos and C. Stein. Approximating disjoint-path problems using packing integer programs. Mathematical Programming, 99(1):63-87, 2004.

44. P. Kolman and C. Scheideler. Simple on-line algorithms for the maximum disjoint paths problem. 13th ACM Symposium on Parallel Algorithms and Architectures (SPAAÕO1), pages 38-47, 2001.

45. M. Krishnamoorthy, A. T. Ernst, and Y. M. Sharaiha. Comparison of algorithms for the degree constrained minimum spanning tree. Journal of Heuristics, pages 587-611, 2001.

46. R. M. Krishnaswamy and K. N. Sivarajan. Algorithms for routing and wavelength assignment based on solutions of LP-relaxations. IEEE Commun. Lett., 5(10):435-437, 2001.

47. K. Lee, K. Kang, T. Lee, and S. Park. An optimization approach to routing and wavelength assignment in wdm all-optical mesh networks without wavelength conversion. ETRI Journal, 24(2):131-141, 2002.

48. C. P. Low. A fast search algorithm for the quorumcast routing problem. Information Processing Letters, 66:87-92, 1998.

49. B. Mukherjee. Optical WDM Networks. Springer, 2006.

50. E. Nardelli and G. Proietti. Finding all the best swaps of a minimum diameter spanning tree under transient edge failures. Journal of Graph Algorithms and Applications vol. 5, no. 5, pages 39-57, 2001.

51. T. Noronha and C. Ribeiro. Routing and wavelength assignment by partition coloring. European Journal of Operational Research, 171(3):797-810, 2006.

52. A. Ozdaglar and D. Bersekas. Routing and wavelength assignment in optical networks. IEEE/ACM Transactions on Networking, 11(2):259-272, 2003.

53. Q. D. PHAM. LS(Graph): A constrained-based local search framework for constrained optimum tree and path problems on graphs, PhD thesis. Université catholique de Louvain, 2011.

54. Q. D. Pham, Y. Deville, and P. V. Hentenryck. Constraint-based local search for constrained optimum paths problems. In Proceedings of the seventh International Conference on Integration of Artificial Intelligence and Operations Research techniques in Constraint Programming, CPAIOR'2010, pages 267-281, 2010. 
55. R. Ramaswami and K. Sivarajan. Routing and wavelength assignment in all-optical networks. IEEE/ACM Trans. Network, 3(5):489-500, 1995.

56. M. Reimann and M. Laumanns. A hybrid aco algorithm for the capacitated minimum spanning tree problem. Proceedings of First International Workshop on Hybrid Metaheuristics, pages 1-10, 2004.

57. D. D. Sleator and R. E. Tarjan. A data structure for dynamic trees. JCSS, 26(3):362-391, 1983.

58. D. D. Sleator and R. E. Tarjan. Self-adjusting binary search trees. Journal of the Association for Computing Machinery, 32(3):652-686, 1985.

59. R. E. Tarjan and R. F. Werneck. Self-adjusting top trees. Proceedings of the 16th Annual ACM-SIAM Symposium on Discrete Algorithms (SODA), pages 813-822, 2005.

60. R. E. Tarjan and R. F. Werneck. Dynamic trees in practice. Journal of Experimental Algorithmics (JEA), 14(Article No.: 5), 2009.

61. M. Tornatore, G. Maier, and A. Pattavina. Wdm network optimization by ilp based on source formulation. In IN PROCEEDINGS OF IEEE INFOCOM, pages 1813-1821, 2002.

62. P. Van Hentenryck and L. Michel. Constraint-based local search. The MIT Press, London, England, 2005.

63. B. Wang and J. C. Hou. An efficient qos routing algorithm for quorumcast communication. Computer Networks Journal, 44(1):43-61, 2004.

64. Y. Ye, T. Chai, T. Cheng, and C. Lu. Algorithms for the design of wdm translucent optical networks Optics Express, 11(22).

65. E. Yetginer, Z. Liu, and G. N. Rouskas. RWA in WDM Rings: An efficient formulation based on maximal independent set decomposition. In The 17th IEEE Workshop on Local and Metropolitan Area Networks (IEEE LANMAN 2010), 2010.

66. M. Zachariasen. Local Search for the Steiner Tree Problem in the Euclidean Plane. European Journal of Operational Research, 119:282-300, 1999.

67. H. Zang, J. P. Jue, and B. Mukherjee. A review of routing and wavelength assignment approaches for wavelength- routed optical wdm networks. Optical Networks Magazine, 1(1):47-60, 2000.

\section{A Appendix}

This Appendix presents the proofs of above propositions.

\section{A.1 Proof of Proposition 1}

Proof The proof is divided into two phases:

1. We show that if the conditions (1) and (2) are satisfied, then path $h_{t r^{\prime}}(s) \neq$ path $_{t r}(s)$.

The condition (1) ensures that the selected edge $e^{\prime}$ satisfying the condition (2) always exist. It is easy to see that $e^{\prime}$ belongs to path $_{t r}(s)$ and this edge is removed from that path after taking $r e p\left(t r, e^{\prime}, e\right)$.

That means $e^{\prime}$ does not belong to path $_{t r^{\prime}}(s)$. Hence, $\operatorname{path}_{t r^{\prime}}(s) \neq$ path $_{t r}(s)$.

2. We now show that if $\operatorname{path}_{t r^{\prime}}(s) \neq \operatorname{path}_{t r}(s)$, then the conditions (1) and (2) are satisfied.

We prove this by refutation. Suppose that $s u=s v$. We denote $r=s u=s v$ and $r_{1}=n c a_{t r}(u, v)$. Because $r$ Dom $_{t r} u$ and $r \operatorname{Dom}_{t r} v$, we have $r \operatorname{Dom}_{t r} n c a_{t r}(u, v)=r_{1}$ (3).

We now show that path $h_{t r}(u, v)$ does not contain any edges that belong to $\operatorname{path}_{t r}(s)$

- If $\operatorname{path}_{t r}\left(u, r_{1}\right)$ contains an edge $(x, y)$ (where $\left.y=f a_{t r}(x)\right)$ of path $\operatorname{tr}_{t r}(s)$, then we have $x$ Dom $_{t r} u$ and $x$ Dom $_{t r} s$. Hence, $x \operatorname{Dom}_{t r} n_{c a} r(s, u)=r$ (4). Otherwise, $(x, y) \in$ path $_{t r}\left(u, r_{1}\right)$, so $r_{1} D_{o m_{t r}} y$, and we have $r \operatorname{Dom}_{t r} y$ (because $r \operatorname{Dom}_{t r} r_{1}$ ) that means $r D_{\text {om }} f a_{t r}(x)$ (5). We see that (4) conflicts with (5). From that, we have the fact that path $_{t r}\left(u, r_{1}\right)$ does not contain any edges of $\operatorname{path}_{t r}(s)$.

- In the same way we can show that $\operatorname{path}_{t r}\left(v, r_{1}\right)$ does not contain any edges of $p a t h_{t r}(s)$. From that, we have $p a t h_{t r}(u, v)$ which is actually the concatenation of $p_{a t h}\left(u, r_{1}\right)$ and $p_{t h} h_{t r}\left(v, r_{1}\right)$ does not contain any edges of path $_{t r}(s)$.

$e^{\prime}$ is a replacable edge that belongs to $\operatorname{path}_{t r}(u, v)$. So after the replacement is taken, no edge of $\operatorname{path}_{t r}(s)$ is removed. Hence, the path from $s$ to the root of the tree does not change, that means $\operatorname{path}_{t r^{\prime}}(s)=\operatorname{path}_{t r}(s)$ (this conflicts with the hypothesis that path $t_{t r^{\prime}}(s) \neq p_{t h} h_{t r}(s)$ ). So we have $s u \neq s v$. 
We now suppose that $e^{\prime}$ (the edge to be removed) does not belong to pathtr $(s u, s v)$. We can see easily that the path from $u$ to $v$ on $\operatorname{tr}\left(\operatorname{path}_{t r}(u, v)\right.$ ) is composed by the path from $u$ to $s u$, the path from $s u$ to $s v$ and the path from $s v$ to $v$ on $t r$. So after the replacement is taken, no edge of $\operatorname{path}_{t r}(s)$ is removed. Hence, $\operatorname{path}_{t r^{\prime}}(s)=\operatorname{path}_{t r}(s)$ (this conflicts with the hypothesis). So we have $e^{\prime} \in$ path $_{t r}(s u, s v)$

\section{A.2 Proof of Proposition 2}

Proof The proposition is proved by showing how to generate that instance $t r^{k}$. This can be done by Algorithm 9. The idea is to travel the sequence of nodes of $\mathcal{P}$ on the current tree $t r$. Whenever we get stuck (we cannot go from the current node $x$ to the next node $y$ of $\mathcal{P}$ by an edge $(x, y)$ on $\operatorname{tr}$ because $(x, y)$ is not in $t r)$, we change $t r$ by replacing $(x, y)$ by a replacable edge of $(x, y)$ that is not traversed. The edge $(x, y)$ in line 7 is a replacing edge of $t r$ because this edge is not in $t r$ but it is an edge of $g$. Line 8 chooses a replacable edge $e o$ of $e i$ that is not in $S$. This choice is always successfully done because the set of replacable edges of $e i$ that are not in $S$ is not null (at least an edge $\left(y, f a_{t r}(y)\right)$ belongs to this set). Line 9 performs the move that replaces the edge eo by the edge $e i$ on $t r$. So Algorithm 9 always terminates and returns a rooted spanning tree $t r$ inducing $\mathcal{P}$. Variable $S$ (line 1) stores the set of traversed edges.

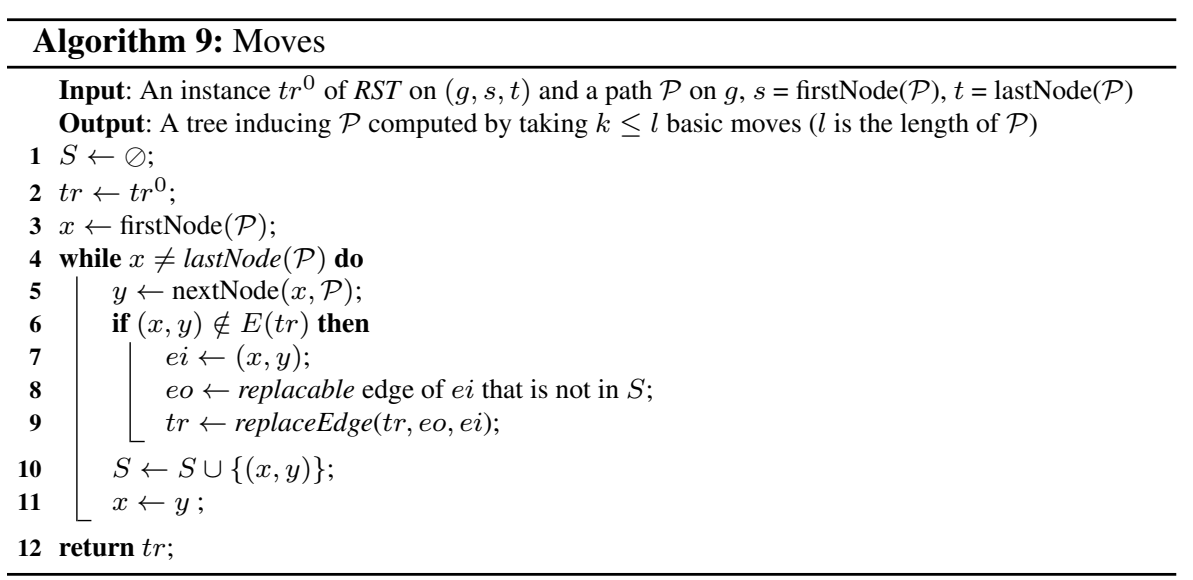

\section{A.3 Proof of Proposition 3}

Proof All sequences of these basic moves are executable and the final results have the same set of edges $E(t r) \backslash\left\{e o_{1}, e o_{2}, \ldots, e o_{k}\right\} \cup\left\{e i_{1}, e i_{2}, \ldots, e i_{k}\right\}$. Thus the result trees of all execution sequences are the same.

\section{A.4 Proof of Proposition 4}

Proof Let $x=n c a_{t r}\left(u_{1}, v_{2}\right), s v_{1}=n c a_{t r}\left(s, v_{1}\right), s u_{1}=n c a_{t r}\left(s, u_{1}\right), s v_{2}=n c a_{t r}\left(s, v_{2}\right)$, $s u_{2}=n c a_{t r}\left(s, u_{2}\right)$. Because $s u_{1} D_{o m_{t r}} s v_{1}, s v_{2} D_{o m_{t r}} s u_{1}, s u_{2} D_{o m_{t r}} s v_{2}, e_{1}^{\prime}$ belongs to 
path $_{t r}\left(s v_{1}, s u_{1}\right)$ and $e_{2}^{\prime}$ belongs to $\operatorname{path}_{t r}\left(s v_{2}, s u_{2}\right)$, we have $e_{1}^{\prime} \neq e_{2}^{\prime}$. Otherwise, $e_{2} \operatorname{Dom}_{(t r)} e_{1}$ and these two edges are not in $t r$, whereas $e_{1}^{\prime}$ and $e_{2}^{\prime}$ are in $t r$. So $e_{1}, e_{1}^{\prime}, e_{2}, e_{2}^{\prime}$ are all different.We will show that the sequence: $\operatorname{rep}\left(\operatorname{tr}, e_{1}^{\prime}, e_{1}\right), \operatorname{rep}\left(\operatorname{tr}, e_{2}^{\prime}, e_{2}\right)$ is feasible as follows:

Suppose that $v_{1}^{\prime}, u_{1}^{\prime}$ are endpoints of $e_{1}^{\prime}$ such that $u_{1}^{\prime}=f a_{t r}\left(v_{1}^{\prime}\right)$ and let $t r_{1}=\operatorname{rep}\left(t r, e_{1}^{\prime}, e_{1}\right)$. We have that:
$s u_{1}$ Dom $_{t r} u_{1}^{\prime}$
$s u_{2}$ Dom $_{t r} u_{1}^{\prime}$
$s v_{2}$ Dom $_{t r} u_{1}^{\prime}$

It is straightfoward to find that $\overline{T_{t r}}\left(v_{1}^{\prime}\right)$ does not change after taking $\operatorname{rep}\left(t r, e_{1}^{\prime}, e_{1}\right)$. We can also find that $u_{1}, v_{2}, u_{2}$ must belong to $\overline{T_{t r}}\left(v_{1}^{\prime}\right)$ (if not, $u_{1}, v_{2}, u_{2}$ must belong to $T_{t r}\left(v_{1}^{\prime}\right)$, thus $n c a_{t r}\left(s, u_{1}\right)$, $n c a_{t r}\left(s, v_{2}\right), n c a_{t r}\left(s, u_{2}\right)$ are dominated by $v_{1}^{\prime}$, hence this conflicts with (1), (2), (3)). Thus $n c a_{t r_{1}}\left(s, u_{2}\right)$ $=n c a_{t r}\left(s, u_{2}\right)=s u_{2}$ and $n c a_{t r_{1}}\left(u_{1}, v_{2}\right)=n c a_{t r}\left(u_{1}, v_{2}\right)=x$. Moreover, from the property 1 , we have $n c a_{t r_{1}}\left(s, v_{2}\right)=n c a_{t r_{1}}\left(u_{1}, v_{2}\right)=x(4)$.

Due to the fact that $s v_{2} D_{o m_{t}} s u_{1}$ and $s u_{1} D_{0 m_{t r_{1}}} u_{1}$, we have $s v_{2} D_{o m_{t r_{1}}} u_{1}$ (5). From the fact that $s v_{2} D_{o m_{t r_{1}}} v_{2}$ and $s v_{2} D_{o m_{r_{1}}} u_{1}$, we have $s v_{2} \operatorname{Dom}_{t r_{1}} n c a_{t r_{1}}\left(u_{1}, v_{2}\right)=x(6)$. We have $e_{2}^{\prime}$ belongs to path $t r\left(s v_{2}, s u_{2}\right)$ (7). From (6) and (7) we have that $e_{2}^{\prime}$ belongs to $p a t h_{t r_{1}}\left(x, s u_{2}\right)$ (8). From (4) and (8), we have $e_{2}^{\prime} \in \operatorname{path}_{t r^{1}}\left(n c a_{t r^{1}}\left(s, v_{2}\right), n c a_{t r^{1}}\left(s, u_{2}\right)\right.$. That means $e_{2}^{\prime}$ is still a preffered replacable edge of $e_{2}$ on $t r_{1}$. So the sequence $\operatorname{rep}\left(\operatorname{tr}, e_{1}^{\prime}, e_{1}\right), \operatorname{rep}\left(t r, e_{2}^{\prime}, e_{2}\right)$ is feasible.

In similar way, we can prove that the sequence $\operatorname{rep}\left(t r, e_{2}^{\prime}, e_{2}\right), r e p\left(t r, e_{1}^{\prime}, e_{1}\right)$ is also feasible. Hence, two basic moves $\operatorname{rep}\left(\operatorname{tr}, e_{1}^{\prime}, e_{1}\right), \operatorname{rep}\left(t r, e_{2}^{\prime}, e_{2}\right)$ are independent. 